\title{
Density Functional Theories of Hard Particle Systems
}

P. Tarazona ${ }^{1}$, J.A. Cuesta ${ }^{2}$, and Y. Martínez-Ratón ${ }^{2}$

1 Departamento de Física Teórica de la Materia Condensada, Universidad Autónoma de Madrid, E-28049 Madrid, Spain pedro.tarazona@uam.es

2 Grupo Interdisciplinar de Sistemas Complejos (GISC), Departamento de Matemáticas, Universidad Carlos III, Avda. de la Universidad 30, E-28911 Leganés, Spain cuesta@math.uc3m.es, yuri@math.uc3m.es

To the memory of our friend Yasha Rosenfeld, who discovered the Fundamental Measure Theory, making this chapter grow into a thick one.

This chapter deals with the applications of the density functional (DF) formalism to the study of inhomogeneous systems with hard core interactions. It includes a brief tutorial on the fundamentals of the method, and the exact free energy DF for one-dimensional hard rods obtained by Percus. The development of DF approximations for the free energy of hard spheres (HS) is presented through its milestones in the weighted density approximation (WDA) and the fundamental measure theory (FMT). The extensions of these approaches to HS mixtures include the FMT treatment of polydisperse systems and the approximations for mixtures with non-additive core radii. The DF treatment of non-spherical hard core systems is presented within the generic context of the study of liquid crystals phases. The chapter is directed to the potential users of these theoretical techniques, with clear explanations of the practical implementation details of the most successful approximations.

\subsection{Introduction}

The density functional (DF) formalism for classical particles [1] was developed to find out the equilibrium density distribution $\rho(\boldsymbol{r})$ of inhomogeneous systems at interfaces or in the presence of an external potential $V(\boldsymbol{r})$. In most cases, like the layering of fluids against walls or liquids confined in nano-capillaries, the sharpest level of structure in $\rho(\boldsymbol{r})$ comes from the effects of molecular packing, and hence the development of DF theories for hard-core models has been a 
main objective in the field $[2,3]$. The free energy DF for one-dimensional (1D) hard rods (HR), presented by Percus [4] in 1976, provided both an exact case to test the internal relations of the DF formalism and a hint on how to approximate the free energy of three-dimensional (3D) hard spheres (HS) and two-dimensional (2D) hard disk (HD) systems. Over the last decades, there has been an impressive improvement in the quality of these approximations, with milestones in the weighted density approximation (WDA) in the middle 1980s and the fundamental measure theory (FMT) over the 1990s.

After a brief tutorial to the DF formalism, to be skipped by the expert reader, this chapter will first deal with the most successful DF schemes for HS, presenting them in an easy-to-use fashion and comparing their relative advantages and difficulties. We do not intend an exhaustive presentation of the many approaches and variants, which have been developed to describe the DF free energy of these systems, but rather to provide the reader with an easy to follow introduction to the basis and the practical use of some extensively used approaches.

The second part of the chapter will be dedicated to the free energy DF for HS mixtures, for which the FMT appears to be the most natural approximate scheme. Different versions of FMT will be presented and their relative merits compared, including their use for polydisperse systems and the extensions to non-additive HS.

The third part of the chapter will deal with non-spherical hard core systems, describing again the most successful schemes which have been developed to study rigid cores.

\subsection{Brief Tutorial to the Density Functional Formalism}

The DF formalism for systems with classical statistics [1] establishes that for any given temperature $\left(\beta=1 / k_{\mathrm{B}} T\right)$ and form of the pair molecular interaction potential energy, $u\left(\boldsymbol{r}_{i}-\boldsymbol{r}_{j}\right)$, there is a unique intrinsic free energy $\mathcal{F}[\rho]$, which is a functional of the density distribution $\rho(\boldsymbol{r})$ and not of the external potential. The grand canonical equilibrium density distribution for the system in presence of any external potential $V(\boldsymbol{r})$ and in contact with a reservoir of particles at chemical potential $\mu$ is that which minimizes the grand potential energy DF,

$$
\Omega[\rho] \equiv \mathcal{F}[\rho]+\int \mathrm{d} \boldsymbol{r} \rho(\boldsymbol{r})(V(\boldsymbol{r})-\mu),
$$

with respect to all possible functions $\rho(\boldsymbol{r})$. That minimum condition on $\Omega$ may be expressed through the Euler-Lagrange equation

$$
\frac{\delta \Omega[\rho]}{\delta \rho(\boldsymbol{r})} \equiv \frac{\delta \mathcal{F}[\rho]}{\delta \rho(\boldsymbol{r})}+V(\boldsymbol{r})-\mu=0,
$$

in terms of the functional derivative of $\mathcal{F}[\rho]$. 
The explicit knowledge of the (exact or approximate) free energy DF would reduce the equilibrium statistical mechanics of inhomogeneous systems to a problem of functional minimization with respect to the one-particle distribution $\rho(\boldsymbol{r})$, and this would represent a huge simplification with respect to the direct evaluation of the grand partition function,

$$
\Xi=\mathrm{e}^{-\beta \Omega_{0}}=\sum_{N} \frac{\mathrm{e}^{\beta \mu N}}{N ! \Lambda^{3 N}} \int \prod_{i=1}^{N} \mathrm{~d} \boldsymbol{r}_{i} \mathrm{e}^{-\beta U_{N}}
$$

and its use to get the equilibrium density distribution

$$
\rho(\boldsymbol{r})=\left\langle\sum_{i} \delta\left(\boldsymbol{r}-\boldsymbol{r}_{i}\right)\right\rangle=\frac{1}{\Xi} \sum_{N} \frac{\mathrm{e}^{\beta \mu N}}{(N-1) ! \Lambda^{3 N}} \int \prod_{i=1}^{N} \mathrm{~d} \boldsymbol{r}_{i} \mathrm{e}^{-\beta U_{N}} \delta\left(\boldsymbol{r}-\boldsymbol{r}_{i}\right),
$$

where $U_{N}\left(\boldsymbol{r}_{1}, \boldsymbol{r}_{2}, \ldots, \boldsymbol{r}_{N}\right)=\sum_{i j} u\left(\boldsymbol{r}_{i j}\right)+\sum_{i} V\left(\boldsymbol{r}_{i}\right)$ is the total potential energy of $N$ particles, including both the molecular interactions $u(\boldsymbol{r})$ and any external potential $V(\boldsymbol{r})$. The thermal wavelength $\Lambda$ includes in (7.3) and (7.4) the contribution from the momentum integrals, which for classical particles is fully factorized from those over the positions. Since changing the value of $\Lambda$ (i.e. changing the particle mass) produces only a trivial shift of the chemical potential and it does not affect any thermodynamic or structural property, we follow here the usual choice $\Lambda=1$ to waive it out.

\subsubsection{The Ideal Gas and the Excess Free Energy Density Functional}

The classical ideal (non-interacting) gas, $u\left(\boldsymbol{r}_{i j}\right)=0$, provides the simplest pedagogical example of the DF formalism, since the exact sampling of the atomic configurations in (7.3) may be readily computed to get

$$
\Xi_{\text {id }}=\sum_{N=0}^{\infty} \frac{\left(\mathrm{e}^{\beta \mu} Z_{1}\right)^{N}}{N !}=\exp \left(Z_{1} e^{\beta \mu}\right),
$$

in terms of the one-particle partition function

$$
Z_{1}=\int \mathrm{d} \boldsymbol{r} e^{-\beta V(\boldsymbol{r})} .
$$

Therefore, the equilibrium grand potential energy is

$$
\Omega_{\mathrm{id}}[V] \equiv-\frac{1}{\beta} \ln \left(\Xi_{\mathrm{id}}\right)=-\frac{\mathrm{e}^{\beta \mu}}{\beta} \int \mathrm{d} \boldsymbol{r} \mathrm{e}^{-\beta V(\boldsymbol{r})},
$$

and the equilibrium density distribution follows the simple form

$$
\rho_{\mathrm{id}}(\boldsymbol{r})=\mathrm{e}^{\beta(\mu-V(\boldsymbol{r}))} .
$$


These results may be inverted to get, via equation (7.1), the explicit functional form of the ideal gas intrinsic free energy,

$$
\beta \mathcal{F}_{\mathrm{id}}[\rho]=\int \mathrm{d} \boldsymbol{r} \rho(\boldsymbol{r})[\ln \rho(\boldsymbol{r})-1] .
$$

The functional derivative of $\mathcal{F}_{\text {id }}[\rho]$ is

$$
\frac{\delta \mathcal{F}_{\text {id }}[\rho]}{\delta \rho(\boldsymbol{r})}=\frac{1}{\beta} \ln \rho(\boldsymbol{r}),
$$

so that the Euler-Lagrange equation (7.2) leads directly to the equilibrium density (7.8) and its substitution in (7.9) and (7.1) gives back the equilibrium grand potential energy (7.7), which reduces to $\beta \Omega_{\mathrm{id}}=-\int \mathrm{d} \boldsymbol{r} \rho(\boldsymbol{r})=-\langle N\rangle$.

The free energy DF of hard core systems is purely entropic, and it may be split in the ideal gas contribution plus an excess free energy, $\mathcal{F}_{\text {ex }}[\rho]$, to account for the entropy reduction due to the non-overlap of the molecular cores. Hence, it is usually written in $k_{\mathrm{B}} T$ units as

$$
\beta \mathcal{F}[\rho]=\beta \mathcal{F}_{\text {id }}[\rho]+\beta \mathcal{F}_{\text {ex }}[\rho] \equiv \int \mathrm{d} \boldsymbol{r}\left\{\Phi_{\text {id }}(\rho(\boldsymbol{r}))+\Phi([\rho] ; \boldsymbol{r})\right\},
$$

with the ideal free energy density as a function, $\Phi_{\text {id }}(\rho)=\rho(\ln \rho-1)$, evaluated at the local density, while the excess contribution is expressed as the volume integral of an excess free energy density $\Phi([\rho] ; \boldsymbol{r})$, which is a function of $\boldsymbol{r}$ and a functional of $\rho(\boldsymbol{r})$. Notice that there could be multiple choices of $\Phi([\rho] ; \boldsymbol{r})$ leading to the same $\mathcal{F}_{\text {ex }}[\rho]$, since there are many ways to separate the total free energy excess of the system in terms of local contributions, and we will see that different approaches to $\mathcal{F}_{\text {ex }}[\rho]$ may give similar results with very different $\Phi([\rho] ; \boldsymbol{r})$.

The development and use of approximations for $\Phi([\rho] ; \boldsymbol{r})$ is the goal of the DF formalism $[2,3]$. The main ingredients to build DF approximations are the results for uniform systems of HS and other hard-core fluids presented in the accompanying chapters of this book. Within the DF formalism, the properties of bulk uniform fluids, with homogeneous density distributions $\rho(\boldsymbol{r})=\rho_{0}$, are associated to the case of null external potential, $V(\boldsymbol{r})=0$, so that the spatial symmetry is recovered in (7.4) from the translational invariance of $U_{N}$. From (7.2), we get that the functional derivative of $\mathcal{F}[\rho]$, evaluated at $\rho(\boldsymbol{r})=\rho_{0}$, has to be constant and equal to the equilibrium chemical potential of the system, while $\Omega\left[\rho_{0}\right]$ and $\mathcal{F}\left[\rho_{0}\right]$ in (7.1) become the extensive thermodynamic potentials $\Omega_{0}$ and $F_{0}$, proportional to the total volume of the system.

\subsubsection{The Correlation Structure of Uniform Systems}

A most interesting result of the DF formalism [1] is the relation between $\mathcal{F}_{\text {ex }}[\rho]$ and the direct correlation function, $c\left(r, \rho_{0}\right)$, of a bulk fluid. This function was originally defined through the Ornstein-Zernike equation, 


$$
h\left(r, \rho_{0}\right)=c\left(r, \rho_{0}\right)+\rho_{0} \int \mathrm{d} \boldsymbol{r}^{\prime} c\left(r^{\prime}, \rho_{0}\right) h\left(\left|\boldsymbol{r}-\boldsymbol{r}^{\prime}\right|, \rho_{0}\right),
$$

in terms of the total correlation function $h\left(r, \rho_{0}\right) \equiv g\left(r, \rho_{0}\right)-1$. For any system with classical statistics, there is an exact DF relation,

$$
\left.\frac{\delta^{2} \beta \mathcal{F}_{\mathrm{ex}}[\rho]}{\delta \rho\left(\boldsymbol{r}_{1}\right) \delta \rho\left(\boldsymbol{r}_{2}\right)}\right|_{\rho_{0}}=-c\left(r_{12}, \rho_{0}\right),
$$

with the second functional derivative of $\mathcal{F}_{\text {ex }}[\rho]$ evaluated at $\rho(\boldsymbol{r})=\rho_{0}$.

The functional Taylor expansion of $\mathcal{F}_{\text {ex }}[\rho]$ in terms of the density difference, $\Delta \rho(\boldsymbol{r})=\rho(\boldsymbol{r})-\rho_{0}$, with respect to a uniform bulk reference system, takes the generic form

$$
\begin{aligned}
\beta \mathcal{F}_{\mathrm{ex}}[\rho]= & \beta F_{\mathrm{ex}}\left(\rho_{0}\right)+\beta \mu_{\mathrm{ex}}\left(\rho_{0}\right) \int \mathrm{d} \boldsymbol{r} \Delta \rho(\boldsymbol{r})- \\
& -\frac{1}{2} \int \mathrm{d} \boldsymbol{r} \mathrm{d} \boldsymbol{r}^{\prime} c\left(\left|\boldsymbol{r}-\boldsymbol{r}^{\prime}\right|, \rho_{0}\right) \Delta \rho(\boldsymbol{r}) \Delta \rho\left(\boldsymbol{r}^{\prime}\right)+\mathcal{O}^{3}(\Delta \rho(\boldsymbol{r})),
\end{aligned}
$$

where the excess free energy, $F_{\text {ex }}\left(\rho_{0}\right)$, and chemical potential $\mu_{\mathrm{ex}}\left(\rho_{0}\right)$, of the bulk liquid are directly obtained from its equation of state, while $c\left(r, \rho_{0}\right)$ may be obtained from the bulk correlation structure. The excellent approximations for these bulk quantities, reviewed in the accompanying chapters of this book, may then give a good approximation to $\beta \mathcal{F}_{\text {ex }}[\rho]$ for systems with weak variations of the density. The drawback of the truncated functional Taylor expansion is that for density distributions having a large constant plateau at a value different from $\rho_{0}$, the local thermodynamics at the plateau is poorly represented beyond the quadratic dependence of $F_{\text {ex }}(\rho)$ around $\rho=\rho_{0}$.

From the opposite point of view, we may take any given DF approximation for $\mathcal{F}_{\text {ex }}[\rho]$ and use it to predict in two different ways the correlation structure of any bulk liquid. The $O Z$ route gets $c\left(r, \rho_{0}\right)$ from $(7.13)$ and then $h\left(r, \rho_{0}\right)$ from (7.12), while for systems with pairwise interactions, the test-particle route uses the pair potential between two molecules $u(r)$ as an "external" potential acting on a bulk fluid from a particle pinned at the origin. The solution of the Euler-Lagrange equation (7.2) would then give the density distribution of the remaining molecules, which is interpreted as $\rho(r) \equiv \rho_{0}[1+h(r, \rho)]$ in terms of the total correlation in the bulk fluid. The exact DF form of $\mathcal{F}[\rho]$, for a given molecular interaction, should give the same result for $h\left(r, \rho_{0}\right)$ along the two routes, but the use of any DF approximation would lead to inconsistent predictions. In particular, for hard-core interactions, the test-particle route would always fulfill the exact requirement $h\left(r, \rho_{0}\right)=-1$ inside the core, while the compressibility route would (in general) fail to satisfy that condition. On the other hand, the sum rule on the total correlation function in the grandcanonical ensemble [5]

$$
\rho_{0} \int \mathrm{d} \boldsymbol{r} h\left(r, \rho_{0}\right)=-1+\rho_{0} k_{\mathrm{B}} T \chi_{T},
$$


in terms of the bulk liquid isothermal compressibility $\chi_{T} \equiv \rho_{0}^{-1}\left(\partial \rho_{0} / \partial p\right)_{T}$ (where $p$ stands for pressure), is always satisfied by the compressibility route to $h\left(r, \rho_{0}\right)$, but not by the test-particle one with $\mathrm{DF}$ approximations for $\mathcal{F}[\rho]$. In general, the degree of inconsistency between the compressibility and the test-particle routes to $h\left(r, \rho_{0}\right)$ gives a quality test for any DF approximation.

\subsubsection{Density Functional Virial Expansion of the Excess Free Energy}

The usual virial expansion for the thermodynamics, and correlation structure, of bulk fluids may be extended to get the generic density expansion of $\mathcal{F}_{\text {ex }}[\rho]$ in systems with pairwise interactions,

$$
\begin{aligned}
\beta \mathcal{F}_{\text {ex }}[\rho]= & \frac{1}{2} \int \mathrm{d} \boldsymbol{r}_{1} \rho\left(\boldsymbol{r}_{1}\right) \int \mathrm{d} \boldsymbol{r}_{2} \rho\left(\boldsymbol{r}_{2}\right) f\left(r_{12}\right)+ \\
& +\frac{1}{6} \int \mathrm{d} \boldsymbol{r}_{1} \rho\left(\boldsymbol{r}_{1}\right) \int \mathrm{d} \boldsymbol{r}_{2} \rho\left(\boldsymbol{r}_{2}\right) \int \mathrm{d} \boldsymbol{r}_{3} \rho\left(\boldsymbol{r}_{3}\right) f\left(r_{12}\right) f\left(r_{23}\right) f\left(r_{31}\right)+\cdots,
\end{aligned}
$$

where $f(\boldsymbol{r})=1-\exp (-\beta u(\boldsymbol{r}))$ is the Mayer function, ${ }^{1}$ with the pairwise potential $u(\boldsymbol{r})$. The expansion for the direct correlation function in a system with uniform density follows from (7.13) and (7.16),

$$
-c\left(r_{12}, \rho_{0}\right)=f\left(r_{12}\right)+\rho_{0} f\left(r_{12}\right) \int \mathrm{d} \boldsymbol{r}_{3} f\left(r_{23}\right) f\left(r_{31}\right)+\cdots .
$$

As expected, the lowest order of the excess free energy DF produced by the interactions between the particles is quadratic in $\rho(\boldsymbol{r})$, while its second functional derivative has a zero density limit $c(r, 0)=-f(r)$. These exact lowdensity results for $\beta \mathcal{F}_{\text {ex }}[\rho]$ provide useful hints for the development of DF approximations.

\subsubsection{The Local Density Approximation and the Gradient Expansion}

The local density approximation (LDA) is the simplest scheme to build a DF approximation for $\beta \mathcal{F}_{\text {ex }}[\rho]$ from the restricted knowledge of the thermodynamics of uniform systems. This DF form approximates the local excess free energy density in (7.11) as a function of the local density, $\Phi_{\mathrm{LDA}}([\rho] ; \boldsymbol{r})=\Phi(\rho(\boldsymbol{r}))$, where $\Phi\left(\rho_{0}\right)$ is the excess free energy per unit volume in a system with uniform density $\rho_{0}$, directly accessible from its equation of state. The ideal gas free energy DF does have exactly the local density form (7.9), but the generic

\footnotetext{
${ }^{1}$ Notice the different sign of this definition with respect to the standard one.
} 
form of the excess $\Phi([\rho] ; \boldsymbol{r})$ depends on the density distribution at different positions, as already observed in the first term of the virial expansion (7.16), so that the LDA can only give acceptable results if $\rho(\boldsymbol{r})$ changes very smoothly over molecular size distances. The LDA description of the HS excess free energy may also be regarded as a coarse grained description of $\rho(\boldsymbol{r})$, when we are interested in its long-ranged aspects rather than in the molecular layering structure [6].

For systems with attractive interactions, there is a systematic, although limited, way to improve the LDA in terms of density gradient expansions [1], assuming that the generic functional $\Phi([\rho] ; \boldsymbol{r})$ is not just a local function of $\rho(\boldsymbol{r})$ but also of its local gradient $\nabla \rho(\boldsymbol{r})$. van der Waals theory for the structure of the liquid surface may be considered as the precursor of that DF approximation [7], well before the DF formalism was established. However, the approach fails from the beginning when applied to systems with hardcore interactions. The non-local dependence of $\Phi([\rho] ; \boldsymbol{r})$ implies a sharp finite range of interference between $\rho(\boldsymbol{r})$ and $\rho\left(\boldsymbol{r}^{\prime}\right)$, associated to the geometry of the molecule, and this cannot be represented as a simple expansion in terms of the local density gradient.

\subsubsection{The Mean Field Approximation}

For systems with very soft molecular potentials, the particles may be assumed to be fully uncorrelated, as in the ideal gas, so that $\mathcal{F}_{\text {ex }}[\rho]$ has got no entropic contribution and is given by the interaction energy

$$
\mathcal{F}_{\text {ex }}[\rho]=\frac{1}{2} \int \mathrm{d} \boldsymbol{r} \mathrm{d} \boldsymbol{r}^{\prime} \rho(\boldsymbol{r}) \rho\left(\boldsymbol{r}^{\prime}\right) u\left(\boldsymbol{r}-\boldsymbol{r}^{\prime}\right) .
$$

This is the mean field approximation (MFA) DF [2], since the functional derivative in (7.2) takes the form

$$
\frac{\delta \mathcal{F}_{\mathrm{ex}}[\rho]}{\delta \rho(\boldsymbol{r})}=\int \mathrm{d} \boldsymbol{r}^{\prime} \rho\left(\boldsymbol{r}^{\prime}\right) u\left(\boldsymbol{r}-\boldsymbol{r}^{\prime}\right),
$$

and it is interpreted as the potential created on a particle at $\boldsymbol{r}$ by the (uncorrelated) mean distribution of particles over the whole system. The MFA may give an accurate description of the full excess free energy for ultra-soft interactions, like those between polymer chains in bad solvents [8], but it is obviously out of question for the description of hard-core interactions with an infinite $u(r)$ inside the molecular cores. The relevance of the MFA in this book comes from the usual treatment of simple liquids to split the interaction potential in a repulsive core, $u_{\text {rep }}(r)$, often described as a reference HS with temperature-dependent diameter and the soft attractive part $u_{\text {att }}(r)$, which may be included through the MFA [2]. Such a simple addition of the molecular packing and the attractive interaction effects is sometimes referred to as a generalized van der Waals approximation, and it is the simplest and very successful approach to the DF of realistic model interactions. 


\subsection{The Exact Density Functional for Hard Rods}

In 1976, Percus [4] presented the exact intrinsic free energy DF for a 1D system of HR. Within the 1D version of (7.11), the excess free energy density for rods of length $\sigma$ is given by

$$
\Phi^{(1 \mathrm{D})}([\rho] ; x)=-\rho(x) \ln \left(1-\int_{x}^{x+\sigma} \mathrm{d} x^{\prime} \rho\left(x^{\prime}\right)\right) .
$$

The asymmetry of this expression, with the integral running only for values of $x^{\prime}$ larger than the local position variable, $x$, comes from the transfer matrix procedure employed to compute it, in which the entropy associated to the insertion of each particle is calculated by fixing the previously inserted particles on its right. Exactly equivalent expressions may be obtained with the opposite asymmetry and with the symmetric combination

$$
\Phi^{(1 \mathrm{D})}([\rho] ; x)=-\frac{\rho(x+\sigma / 2)+\rho(x-\sigma / 2)}{2} \ln [1-\eta(x)],
$$

where the local packing fraction

$$
\eta(x)=\int_{-\frac{\sigma}{2}}^{\frac{\sigma}{2}} \mathrm{~d} x^{\prime} \rho\left(x+x^{\prime}\right)
$$

represents the probability that the point $x$ is covered by a HR.

The explicit DF form of $\beta \mathcal{F}_{\text {ex }}[\rho]$ for HR provides an excellent example to analyze the practical use of the DF formalism and also a most valuable hint to guess the free energy DF approximations for hard bodies in higher dimensions. The minimum principle (7.2), in the presence of any external potential $V(x)$, gives

$$
\ln \rho(x)+\frac{\delta \beta \mathcal{F}_{\mathrm{ex}}[\rho]}{\delta \rho(x)}+\beta V(x)-\beta \mu=0,
$$

with non-local dependence of the excess free energy functional derivative

$$
\frac{\delta \beta \mathcal{F}_{\text {ex }}[\rho]}{\delta \rho(x)}=-\ln \left[1-\eta\left(x+\frac{\sigma}{2}\right)\right]-\int_{x-\sigma}^{x} \mathrm{~d} x^{\prime} \frac{\rho\left(x^{\prime}\right)}{1-\eta\left(x^{\prime}+\frac{\sigma}{2}\right)},
$$

so that $\rho(x)$ cannot be obtained by solving (7.23) independently for each $x$, as in the ideal gas case (7.8). The interactions couple the values of $\rho(x)$ at positions within a rod length $\sigma$, and (7.23) becomes an integral equation to be simultaneously solved for the whole $\rho(x)$, rather than separately for each $x$. Qualitatively, this integral equation is similar to those described below for DF 
approximations for HS and HD, and several numerical techniques have been used either to solve it or equivalently to perform the numerical minimization of $\Omega[\rho]$, as described in the next sections.

The exact direct correlation function of a uniform distribution of HR of length $\sigma$ and homogeneous $1 \mathrm{D}$ density $\rho_{0}$ is directly obtained from the second functional derivative of (7.20) or (7.21),

$$
c\left(\left|x-x^{\prime}\right|, \rho_{0}\right)=-\left.\frac{\delta^{2} \beta \mathcal{F}_{\mathrm{ex}}[\rho]}{\delta \rho(x) \delta \rho\left(x^{\prime}\right)}\right|_{\rho(x)=\rho_{0}}=-\Theta\left(\sigma-\left|x-x^{\prime}\right|\right) \frac{1-\rho_{0}\left|x-x^{\prime}\right|}{\left(1-\eta_{0}\right)^{2}},
$$

where $\Theta(y)$ is the Heaviside step function (=1 if $y>0$ and $=0$ if $y<0$ ), which makes $c\left(|x-x|, \rho_{0}\right)=0$ beyond the contact distance $\left|x-x^{\prime}\right|=\sigma$, and $\eta_{0}=\sigma \rho_{0}$ is the packing fraction of the fluid. Notice that the Mayer function for 1D HR is precisely $f(x)=\Theta(\sigma-|x|)$, so that, as it should be, (7.25) is consistent with the generic density expansion (7.17).

The exact total correlation function $h\left(x, \rho_{0}\right)$ in a system with uniform density $\rho_{0}$ may be consistently obtained from $c\left(x, \rho_{0}\right)$, through the $1 \mathrm{D}$ version of the Ornstein-Zernike equation (7.12), or by the test-particle route, solving (7.24) with $V(x)=0$ for $|x|>\sigma$ and $V(x)=\infty$ for $|x|<\sigma$. The result may be expressed as the infinite sum [9]

$$
\begin{aligned}
h\left(x, \rho_{0}\right)= & 1+\sum_{k=0}^{\infty} \Theta(|x|-(k+1) \sigma) \frac{\rho_{0}^{k}(|x|-(k+1) \sigma)^{k}}{k !\left(1-\eta_{0}\right)^{k+1}} \\
& \times \exp \left\{-\frac{\rho_{0}(|x|-(k+1) \sigma)}{1-\eta_{0}}\right\},
\end{aligned}
$$

with each term in the sum representing the contribution from the successive neighbors of the particle centered at $x=0$ (which in a 1D system with hard core interactions are forced to keep the order along the line). As in other problems in statistical physics, this $1 \mathrm{D}$ ordering is the key element to get the exact functional form of $\beta \mathcal{F}_{\text {ex }}[\rho]$, and it is clearly absent in higher dimensions. Early attempts $[10,11]$ to extend the DF form (7.21) to approximate the free energy DF of 3D HS were not very successful, and the hints offered by 1D system were of little use for the design of DF approximations in two and three dimensions, until the advent of the most recent family of DF approximations based on Rosenfeld's FMT [12, 13] (that will be introduced in Sect. 7.5).

\subsection{The Weighted Density Approximation}

In 1980, Nordholm et al. [14] presented a theory for the free energy of an inhomogeneous HS fluid which inspired a whole family of DF approximations. The most successful members of that family had come to share the name of 
WDA, originally applied in the context of DF theory for the exchange and correlation energy of electronic systems ${ }^{2}$ [15].

The common feature of the WDAs for classical fluids is to approximate the local free energy DF by $\Phi_{\mathrm{WDA}}[\rho, \boldsymbol{r}]=\rho(\boldsymbol{r}) \psi(\bar{\rho}(\boldsymbol{r}))$, where $\psi\left(\rho_{0}\right) \equiv \Phi\left(\rho_{0}\right) / \rho_{0}$ is the excess free energy per particle of a bulk system with uniform density $\rho_{0}$ and $\bar{\rho}(\boldsymbol{r})$ is a weighted density to represent a sampling of the density around the point $\boldsymbol{r}$ through the convolution

$$
\bar{\rho}(\boldsymbol{r})=\int \mathrm{d} \boldsymbol{r}^{\prime} \rho\left(\boldsymbol{r}+\boldsymbol{r}^{\prime}\right) w\left(r^{\prime}, \bar{\rho}(\boldsymbol{r})\right),
$$

with a normalized weight function, $w(r, \rho)$, which may be assumed to depend on the local value of $\bar{\rho}(\boldsymbol{r})$. The choice of that weight function is the key element of the WDA because it determines the non-local dependence of $\mathcal{F}_{\text {ex }}[\rho]$. Notice that the LDA is recovered with a delta-function weight, $w(r)=\delta(\boldsymbol{r})$. The MFA may also be cast into the WDA form by taking the weight function $w(r)=$ $u(r) / u_{0}$, with the pair potential normalized by its total volume integral, $u_{0}$, and the linear function $\psi\left(\rho_{0}\right)=u_{0} \rho_{0} / 2$ for the excess free energy per particle. The use of non-local weights and non-linear functions $\psi\left(\rho_{0}\right)$ made the WDA a very successful DF scheme for HS and other hard-core particles.

Nordholm et al. [14] proposed to use the HS Mayer function to account for that non-local dependence, together with the simplest excluded volume approximation for $\psi(\rho)=-\ln \left(1-2 \pi \sigma^{3} \rho / 3\right)$. The use of more accurate equations of state and the exploration of other analytical forms for $w(r)$ paved the road [16] to the crucial element of the WDA: the (density dependent) weight function $w\left(r, \rho_{0}\right)$ may be tailored, through the relationship (7.13), to reproduce any given approximation for the direct correlation function of the bulk liquid, so that DF approximations for $\mathcal{F}_{\text {ex }}[\rho]$ may be systematically built, using as ingredients the equation of state and the correlation structure of the HS liquid. The resulting functionals are much more effective than the plain functional Taylor expansion (7.16) built with the same ingredients. The price to pay with respect to the earlier density-independent weight functions is that (7.27) becomes an equation for $\bar{\rho}(\boldsymbol{r})$ which has to be solved at every point $\boldsymbol{r}$ and for every density distribution $\rho(\boldsymbol{r})$ arising along the numerical procedure to minimize $\Omega[\rho]$. In practice, this difficulty may be temperated assuming a simplified polynomial dependence [17]

$$
w(r, \rho)=w_{0}(r)+\rho w_{1}(r)+\rho^{2} w_{2}(r)+\cdots,
$$

so that the evaluation of $\bar{\rho}(\boldsymbol{r})$ for a given $\rho(\boldsymbol{r})$ requires a few plain convolutions to get the weighted density components

\footnotetext{
${ }^{2}$ Notice that two different non-local DF schemes were proposed in this work, one under the name of averaged density approximation (ADA) and the other under the name of WDA; but what came to be called as WDA in the context of classical fluids corresponds to the ADA in electronic DF theory. The name ADA was used during some time to refer to the DF approximation in [17].
} 


$$
\bar{\rho}_{\nu}(\boldsymbol{r})=\int \mathrm{d} \boldsymbol{r}^{\prime} \rho\left(\boldsymbol{r}+\boldsymbol{r}^{\prime}\right) w_{\nu}\left(r^{\prime}\right), \quad \nu=0,1,2, \ldots,
$$

and the solution of the algebraic equation

$$
\bar{\rho}(\boldsymbol{r})=\bar{\rho}_{0}(\boldsymbol{r})+\bar{\rho}_{1}(\boldsymbol{r}) \bar{\rho}(\boldsymbol{r})+\bar{\rho}_{2}(\boldsymbol{r}) \bar{\rho}(\boldsymbol{r})^{2}+\cdots .
$$

The normalization of $w(r, \rho)$, imposed so that $\bar{\rho}$ equals the bulk density in a homogeneous system, implies

$$
\int \mathrm{d} \boldsymbol{r} w_{0}(\boldsymbol{r})=1, \quad \int \mathrm{d} \boldsymbol{r} w_{\nu}(\boldsymbol{r})=0, \quad \nu>0 .
$$

The form of the first elementary weight functions, $w_{\nu}(r)$, may be obtained to reproduce the exact first two terms of the exact density expansions (7.16) and (7.17), which are also exactly given by the Percus-Yevick (PY) approximation for the bulk liquid $c_{\mathrm{PY}}\left(r, \rho_{0}\right)$. The exact zero-order weight function is the normalized step function

$$
w_{0}(r)=\frac{3}{4 \pi \sigma^{3}} \Theta(\sigma-r)
$$

employed in the preliminary explorations of the approach $[14,16]$, while the exact $w_{1}(r)$ is the solution to the integral equation

$$
\begin{aligned}
& \frac{10 \pi}{3} \sigma^{3} w_{0}(r)+8 w_{1}(r)+\frac{5 \pi}{3} \sigma^{3} \int \mathrm{d} \boldsymbol{r}^{\prime} w_{0}\left(r^{\prime}\right) w_{0}\left(\left|\boldsymbol{r}+\boldsymbol{r}^{\prime}\right|\right) \\
&+8 \int \mathrm{d} \boldsymbol{r}^{\prime} w_{0}\left(r^{\prime}\right) w_{1}\left(\left|\boldsymbol{r}+\boldsymbol{r}^{\prime}\right|\right)=\left[8-6 \frac{r}{\sigma}+\frac{1}{2}\left(\frac{r}{\sigma}\right)^{3}\right] \Theta(\sigma-r) .
\end{aligned}
$$

This equation is solved in Fourier space as

$$
\hat{w}_{1}(k)=\frac{\pi}{6} \sigma^{3} \frac{\hat{f}(k)-20 \hat{w}_{0}(k)-10 \hat{w}_{0}(k)^{2}}{8\left[1+\hat{w}_{0}(k)\right]},
$$

where, using the notation $q=k \sigma$, we have

$$
\begin{aligned}
\hat{w}_{0}(k) & =\frac{3}{q^{3}}(\sin q-q \cos q), \\
\hat{f}(k) & =\frac{288}{q^{6}}\left[1+q^{2}-\left(1+\frac{q^{2}}{2}+\frac{5 q^{4}}{24}\right) \cos q-q\left(1+\frac{q^{2}}{6}\right) \sin q\right]
\end{aligned}
$$

Hence, the function $w_{1}(r)$ is obtained by the inverse Fourier transform,

$$
w_{1}(r)=\frac{\sigma^{3}}{12 \pi r} \int_{0}^{\infty} \mathrm{d} k k \sin (k r) \frac{\hat{f}(k)-20 \hat{w}_{0}(k)-10 \hat{w}_{0}(k)^{2}}{8\left[1+\hat{w}_{0}(k)\right]},
$$

which may be integrated numerically to get the result represented in Fig. 7.1. The weight function $w_{1}(r)$ has a core region, for $r \leq \sigma$, with negative radial 


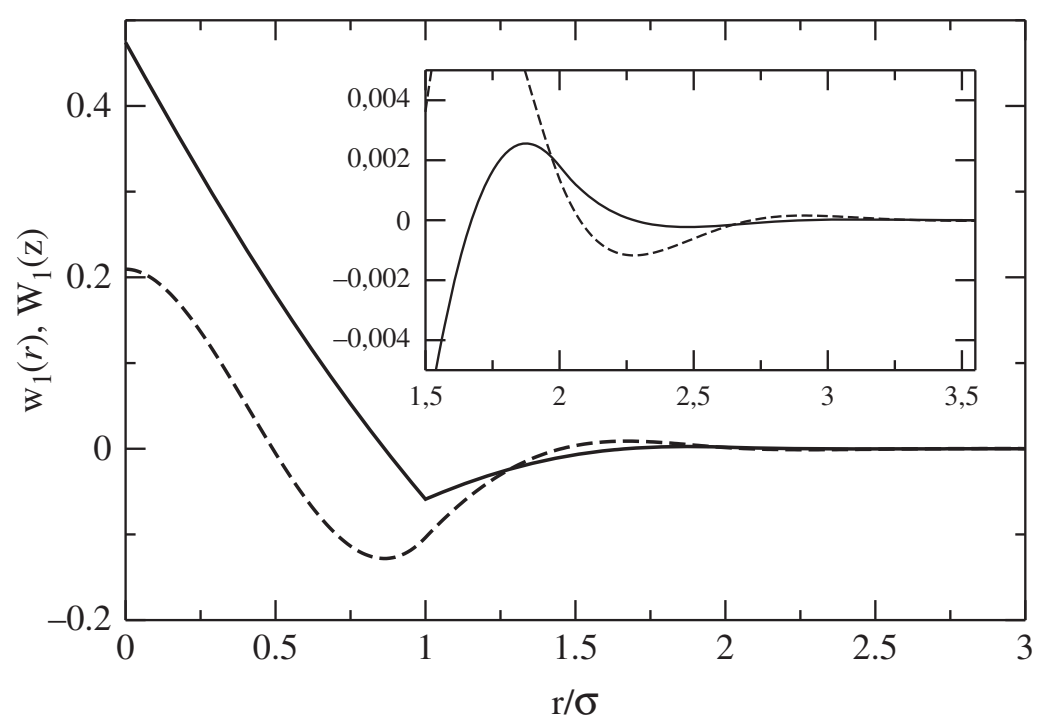

Fig. 7.1. The full line gives the radial weight function $w_{1}(r)$ and the broken line the function $W_{1}(z)$, defined in Eq. (7.42), to be used in problems with planar symmetry. The inset shows the oscillating tails of both functions

slope and an oscillating tail for $r>\sigma$. The shape of $w_{1}(r)$ already indicates that, in order to reproduce the direct correlation function of a dense HS liquid, the WDA representation of $\mathcal{F}_{\text {ex }}[\rho]$ requires a delicate choice of the weighting function.

The WDA proposed under this name by Curtin and Ashcroft in 1985 [18] makes formally consistent use of the PY approximation both for the thermodynamics, $\psi_{\mathrm{PY}}(\rho)$, and for the direct correlation function $c_{\mathrm{PY}}(r, \rho)$; here we will refer to it as PY-WDA. The slightly earlier version, proposed by Tarazona [17], uses (7.29) with the exact forms for $w_{0}(r)$ and $w_{1}(r)$ but truncates the expansion at second order with a purely empirical weight [19]

$$
w_{2}(r)=\frac{5 \pi \sigma^{3}}{24}\left[1-2 \frac{r}{\sigma}+\frac{5}{6}\left(\frac{r}{\sigma}\right)^{2}\right] \Theta(\sigma-r)
$$

to obtain a global good fit to $c_{\mathrm{PY}}\left(r, \rho_{0}\right)$ over the entire range of liquid densities. This approximation is in the same spirit as the one that leads to the semiempirical Carnahan-Starling (CS) equation of state, which does not improve on the PY result for the virial expansion but produces quasi-exact results for the pressure of the HS fluid at any density (see Chap. 3). The CS equation of state was used by Tarazona to fix

$$
\psi_{\mathrm{CS}}(\rho)=\frac{4 \eta-3 \eta^{2}}{(1-\eta)^{2}}
$$

with the packing fraction $\eta=\pi \sigma^{3} \rho / 6$, so that we will refer to that DF approach as CS-WDA. From a practical point of view, the CS-WDA truncation of (7.29) at second order provides a simple analytical solution for (7.27) in terms of the three direct convolutions of the density distribution, $\bar{\rho}_{0}(r), \bar{\rho}_{1}(r)$ 
and $\bar{\rho}_{2}(r)$. The applications of the PY-WDA may also be carried out with some simplified prescription for $w(r, \rho)$, similar to (7.28) and (7.29). Both versions require, in practice, a truncation of the range of $w(r, \rho)$ to a few molecular diameters.

Notice that in any WDA version there are density distributions for which the non-linear equation (7.27) has none or more than one solution. In the CS-WDA, the lack of solutions corresponds to having a negative discriminant, $\left(1-\rho_{1}(\boldsymbol{r})\right)^{2}-4 \rho_{0}(\boldsymbol{r}) \rho_{2}(\boldsymbol{r})<0$, while for a positive discriminant we have to select the appropriate root. In the PY-WDA, the multiplicity or lack of solutions cannot be controlled in an analytical way, but they can still appear along the numerical minimization. It was implicitly assumed that density profiles leading to no positive solution for (7.27) were unphysical, and they may be discarded within the minimization of $\Omega[\rho]$. Whenever (7.27) had more than one positive solution, the physical one is that closest to $\bar{\rho}_{0}(\boldsymbol{r})$. In the CS-WDA, this corresponds to

$$
\bar{\rho}(r)=\frac{2 \bar{\rho}_{0}(r)}{1-\bar{\rho}_{1}(r)+\sqrt{\left[1-\bar{\rho}_{1}(r)\right]^{2}-4 \bar{\rho}_{0}(r) \bar{\rho}_{2}(r)}} .
$$

Both the PY-WDA and the CS-WDA have been extensively applied in the last twenty years to many problems concerning inhomogeneous distributions of HS. Several variants, like splitting $\psi(\bar{\rho})$ in several components, each with a different elementary weight function, have been explored, some of them with similarly good results but with no significant improvement over the whole range of analyzed problems. Therefore, the two 1985 versions have become the standard forms of the WDA. The remaining part of this section presents the application of the CS-WDA to two problems of interest: the density profile $\rho(z)$ of a HS fluid against a flat wall potential $V(z)$ and the description of the HS crystal as a self-stabilized inhomogeneity of the HS fluid. In general, the results with the PY-WDA are fairly close to those of the CS-WDA, so we will only point out their more important differences.

\subsubsection{Hard Sphere Fluid Against a Planar Wall}

In the absence of any kind of symmetry breaking effect, the density distribution of a HS fluid near a planar wall potential $V(z)$ should depend only on the distance to the wall, which we take as the $z$ coordinate. The DF minimization of $\Omega[\rho]$ in (7.1) is then restricted to functions $\rho(\boldsymbol{r})=\rho(z)$, so that the numerical problem of minimization is hugely reduced with respect to that of a 3D dependence of $\rho(x, y, z)$. The weighted density inherits the same symmetry, and the elementary weighted densities may be calculated as $1 \mathrm{D}$ convolutions

$$
\bar{\rho}_{\nu}(z)=\int \mathrm{d} z^{\prime} \rho\left(z+z^{\prime}\right) W_{\nu}\left(z^{\prime}\right),
$$


where the functions

$$
W_{\nu}(z) \equiv \int_{-\infty}^{\infty} \int_{-\infty}^{\infty} \mathrm{d} x \mathrm{~d} y w_{\nu}\left(\sqrt{x^{2}+y^{2}+z^{2}}\right)=2 \pi \int_{|z|}^{\infty} \mathrm{d} r r w_{\nu}(r)
$$

can be analytically obtained for $\nu=0$,

$$
W_{0}(z)=\frac{3}{4 \sigma^{3}}\left(\sigma^{2}-z^{2}\right) \Theta(\sigma-|z|)
$$

and $\nu=2$ in $(7.38)$,

$$
W_{2}(z)=\frac{5 \pi^{2} \sigma^{5}}{288}\left[1-12\left(\frac{z}{\sigma}\right)^{2}+16\left(\frac{|z|}{\sigma}\right)^{3}-5\left(\frac{z}{\sigma}\right)^{4}\right] \Theta(\sigma-|z|),
$$

while $W_{1}(z)$ may be integrated numerically from (7.37) to get the function represented in Fig. 7.1. For practical purposes, $W_{1}(z)$ is tabulated over the discrete mesh of $z$ used to describe the density profile $\rho(z)$ and usually truncated at $|z|=3.55 \sigma$. The total weighted density $\bar{\rho}(z)$ in the CS-WDA version is then readily obtained from (7.40), and the free energy density $\Phi_{\mathrm{WDA}}([\rho] ; z)=\rho(z) \psi(\bar{\rho}(z))$ plus the ideal gas contribution is integrated along the $z$ axis to get the grand potential energy per unit area

$$
\frac{\beta \Omega[\rho]}{A}=\int \mathrm{d} z \rho(z)[\ln \rho(z)-1+\psi(\bar{\rho}(z))+\beta V(z)-\beta \mu] .
$$

This integral should cover all the region of relevance, where $\rho(z)$ is non-zero, up to the bulk region where $\Phi[\rho ; z]=\Phi\left(\rho_{0}\right)$ is constant. For dense HS fluids near sharp walls, the region of variable $\rho(z)$ to be used in the minimization of (7.45) has to cover $10-20 \sigma$ beyond the point where $V(z)$ vanishes. In determining an appropriate integration interval for (7.45), one has to bear in mind that the region where $\bar{\rho}(z) \neq \rho_{0}$ exceeds the one where $\rho(z) \neq \rho_{0}$ by the maximum distance for which $W_{1}(z) \neq 0$. The bulk fluid contribution to $\Omega[\rho]$ is $\Omega_{0}=-p V$, in terms of the bulk pressure $p\left(\rho_{0}\right)$. A $\beta p$ term may be added to the integral in (7.45) so that the integrand vanishes away from the wall and the integral directly gives $\Delta \Omega[\rho] / A=(\Omega[\rho]+p V) / A=\gamma$, i.e. the surface tension of the wall-fluid interface.

The Euler-Lagrange equation (7.2) takes, in the WDA, the form

$$
\ln \rho(z)+\psi(\bar{\rho}(z))+\int \mathrm{d} z^{\prime} \rho\left(z^{\prime}\right) \psi^{\prime}\left(\bar{\rho}\left(z^{\prime}\right)\right) \frac{\delta \bar{\rho}\left(z^{\prime}\right)}{\delta \rho(z)}+\beta V(z)=\beta \mu
$$

where $\psi^{\prime}(\rho) \equiv d \psi(\rho) / d \rho$, and from (7.27),

$$
\frac{\delta \bar{\rho}\left(z^{\prime}\right)}{\delta \rho(z)}=\frac{W\left(\left|z^{\prime}-z\right|, \bar{\rho}\left(z^{\prime}\right)\right)}{1-\int \mathrm{d} z^{\prime \prime} \rho\left(z^{\prime \prime}\right) W^{\prime}\left(\left|z^{\prime}-z^{\prime \prime}\right|, \bar{\rho}\left(z^{\prime}\right)\right)},
$$


and $W^{\prime}(z, \rho)=\partial W(r, \rho) / \partial \rho$. Within the CS-WDA, we get a simple expression for this functional derivative

$$
\frac{\delta \bar{\rho}\left(z^{\prime}\right)}{\delta \rho(z)}=\frac{W_{0}\left(\left|z^{\prime}-z\right|\right)+\bar{\rho}\left(z^{\prime}\right) W_{1}\left(\left|z^{\prime}-z\right|\right)+\bar{\rho}\left(z^{\prime}\right)^{2} W_{2}\left(\left|z^{\prime}-z\right|\right)}{1-\bar{\rho}_{1}\left(z^{\prime}\right)-2 \bar{\rho}\left(z^{\prime}\right) \bar{\rho}_{2}\left(z^{\prime}\right)},
$$

in terms of the same elementary weighted densities which define $\bar{\rho}(z)$. The bulk chemical potential is $\beta \mu=\ln \rho_{0}+\psi\left(\rho_{0}\right)+\rho_{0} \psi^{\prime}\left(\rho_{0}\right)$, so (7.46) is trivially solved within the bulk liquid with $\rho(z)=\rho_{0}$.

The numerical solution of (7.46) is usually obtained with a discretized description of the density profile, $\rho_{i}=\rho\left(z_{i}\right)$, along a regular mesh $\left\{z_{i}\right\}$ over the $z$ axis. If there are $N_{1}$ mesh points in a length $\sigma$, the numerical convolutions to obtain $\bar{\rho}_{\nu}(z)$ (computed with a fast Fourier algorithm [20]) requires a computational cost proportional to $N_{1} \ln N_{1}$, and the evaluation of the integral in (7.46) requires similar convolutions. This should be compared with the computational cost to evaluate the exact expression (7.16) for $\mathcal{F}_{\text {ex }}[\rho]$. The first term of this expression may be exactly cast into the WDA form, $\Phi_{\mathrm{WDA}}[\rho]=\rho(\boldsymbol{r}) \psi(\bar{\rho}(\boldsymbol{r}))$, with $\psi(\rho)=4 \pi \rho \sigma^{3} / 3+\cdots$ and $w(r, \rho)=w_{0}(r)+\cdots$, so that the required number of operations goes also like $N_{1} \ln N_{1}$; but the second term contains a triple integral, which cannot be factorized in terms of a one-center convolution, to get $\bar{\rho}(\boldsymbol{r})$, and an external integral over $\rho(\boldsymbol{r}) \psi(\bar{\rho}(\boldsymbol{r}))$. Therefore, the numerical effort to get the second term in (7.16) is proportional to $N_{1}^{2} \ln N_{1}$. The exponent of $N_{1}$ systematically grows for higher order terms.

This is most relevant to understand the practical limitations in the design of DF approximations, since any application would require many evaluations of $\mathcal{F}_{\text {ex }}[\rho]$ and of its functional derivative to minimize $\Omega[\rho]$. It would be useless to have a prescription for $\mathcal{F}_{\text {ex }}[\rho]$, which requires an unfeasible computational cost, like e.g. the expansion (7.16) extended up to a higher order in $\rho(\boldsymbol{r})$. The successful forms of $\mathcal{F}_{\text {ex }}[\rho]$ are good compromises between the accuracy of the approximation and its computational cost. The free energy for the 1D HR system may be obtained from the computation of the infinite series (7.16), but the exact result (7.20) or (7.21) has a computational cost equivalent to just the first term of the series. Therefore, these DF forms for $\mathcal{F}_{\text {ex }}[\rho]$ are extremely useful rearrangements of (7.16), which achieve an enormous reduction of its computational cost. It is only because of the $1 \mathrm{D}$ character that this can be done exactly; the WDA for 3D HS represents an attempt to estimate the second and higher order terms in (7.16) by means of a one-center convolution with a weight function $w(r, \rho)$. The requirement that $\mathcal{F}_{\mathrm{ex}}^{\mathrm{WDA}}[\rho]$ reproduces the direct correlation function of a bulk liquid implies that $w(r, \rho)$ must be tailored to resum the infinite series (7.16) up to its second functional derivative evaluated at any $\rho(\boldsymbol{r})=\rho_{0}$ and that rather stringent requirement can only be achieved at the price of using a careful tuning of the oscillating tail of $w(r, \rho)$.

The minimization of $\Omega[\rho]$ requires many times the computational cost of its evaluation for a single-density profile. Several numerical methods have been devised to minimize $\Omega[\rho]$ with the discretized description of $\rho(z)$, over the 
$\left\{z_{i}, \rho_{i}\right\}$ mesh. A simple iterative scheme was used in the earliest applications, rewriting (7.46) as

$$
\rho(z)=\rho_{0} \exp \left(-\beta\left[\frac{\delta \mathcal{F}_{\mathrm{ex}}}{\delta \rho(z)}+V(z)-\mu_{\mathrm{ex}}\right]\right)
$$

and using a fixed point iteration, i.e. inserting an initial guess for $\rho(z)$ to evaluate the right-hand side and getting the new estimation for the density profile from the left-hand side. Unless $\rho(z)$ is low everywhere, the convergence of that procedure is very slow. The reason is that, as such, the algorithm seldom converges. In order to make it convergent, the new estimate has to be weight-averaged with the old one, with a stronger weight on the latter, and this severely slows down the iteration. A more efficient method is the use of conjugate gradient techniques, exploring the minimum of $\Omega[\rho]$ along the functional direction set by $\delta \Omega[\rho] / \delta \rho(z)$ and projecting on the subspace which has not been previously explored [20]. In any case, the density profiles like those shown in Fig. 7.2, for HS fluids against a hard wall, present an increasingly strong layering structure for growing $\rho_{0}$, which reflects the ordered packing of the spheres induced by the planar wall. The functional dependence of $\mathcal{F}_{\text {ex }}^{\mathrm{WDA}}[\rho]$ is sharp enough to reproduce those layered structures as the minimum of $\Omega[\rho]$. The rather costly numerical minimization process is intrinsic to the strongly non-local dependence of the free energy with $\rho(z)$.

Compared with computer simulations [21], the qualitative results obtained either with the CS-WDA or the PY-WDA are similarly good; only for very high-bulk densities a difference in favor of the CS-WDA is observed for the contact value of the density at the wall, directly related to the bulk pressure
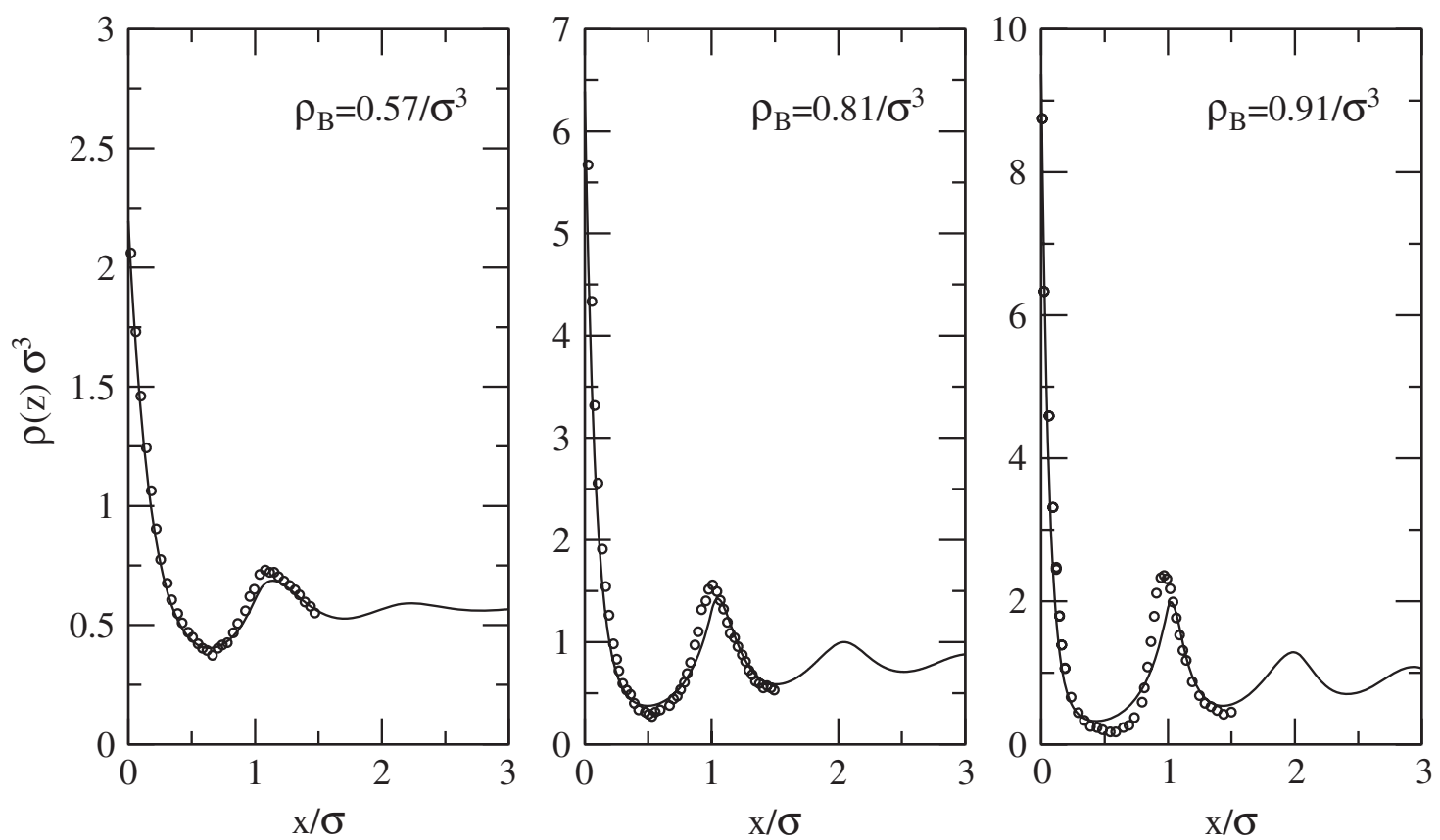

Fig. 7.2. Density profiles of the HS fluid at a hard wall, for three values of the bulk density. The lines are the results of CS-WDA, and the circles are the computer simulations by Snook and Henderson [21] 
through the mechanical equilibrium balance $\rho(0)=\beta p$, which is consistently given by any DF approximation beyond the LDA [2]. Therefore, the use of the CS bulk equation of state gives quasi-exact values for $\rho(0)$, while the PY-WDA version tends to overestimate that contact value. Within the oscillatory part of $\rho(z)$, the two DF approximations give similar results, in reasonably good agreement with the computer simulations, over the full range of fluid densities (notice that the highest $\rho_{0}$ in Fig. 7.2 is only slightly below the freezing value). For $\rho_{0}>0.8 \sigma^{-3}$, the shape and position of the second peak in the layering structure shows some discrepancies with the computer simulation results, but the overall quality of the WDA results is quite satisfactory and generally better than those obtained from the application of integral equation approximations with the wall-particle representation. Moreover, the WDA (as any DF approximation) provides consistent results for the density profiles and for the free energy of the system, which is not accessible from the integralequation approach to $\rho(z)$. The results for the wall-fluid surface tension are presented in Fig. 7.3, and they have a very good agreement with the best computer simulations [22].

The consistent access to the density structure and the thermodynamics is a major advantage of the DF formalism for the study of phase transitions in surface or confined systems, like the problems of wetting and capillary condensation. These problems have been extensively explored $[2,19]$ for models with reference HS cores plus attractive tails, within the generalized van der Waals DF treatment described in Sect. 7.2.5.

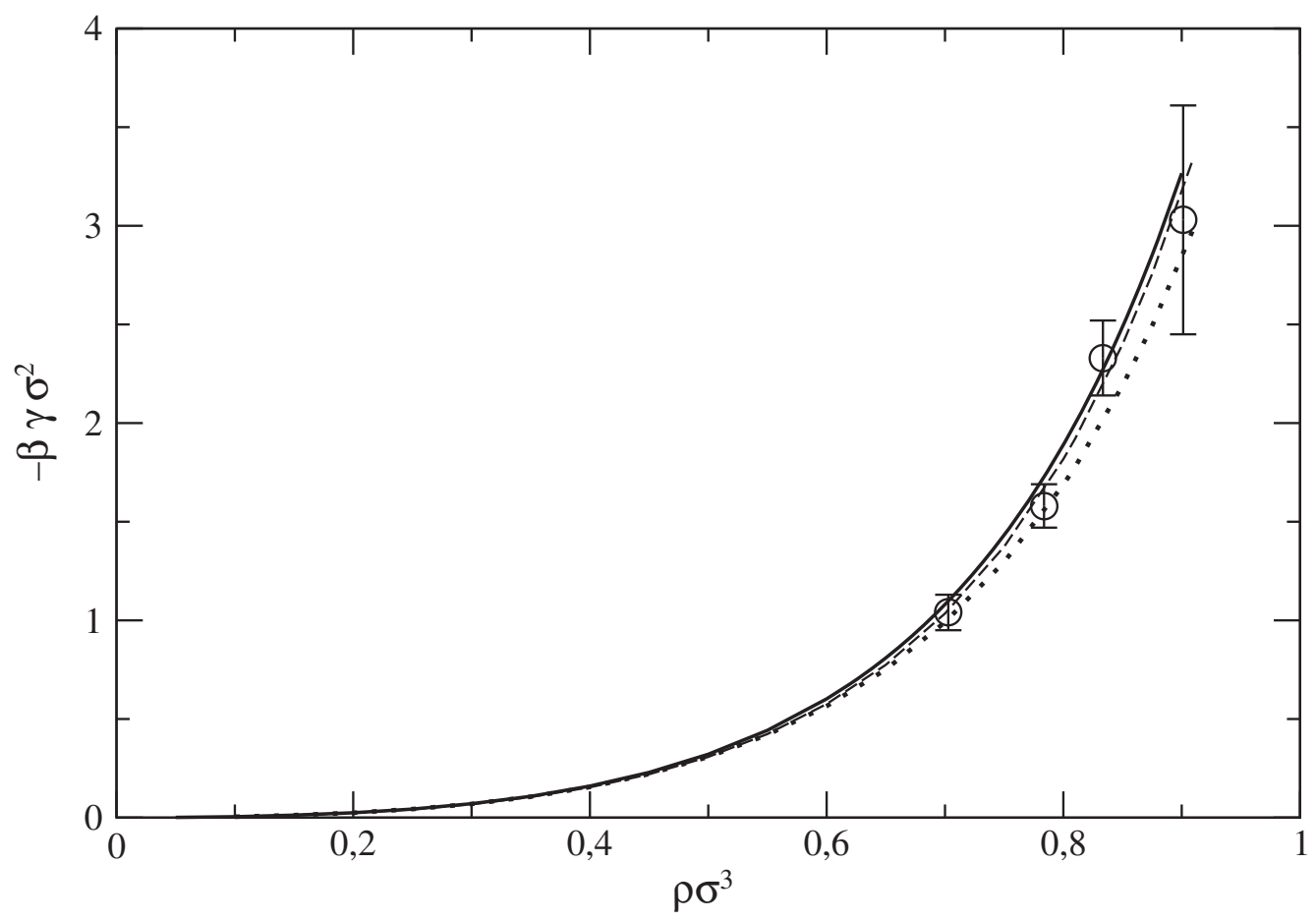

Fig. 7.3. Surface tension (with the sign changed) in $k T / \sigma^{2}$ units for the HS fluid at a hard wall. The circles with error bars are the results from the computer simulations by Henderson and van Swol [22]. The dotted line is the result of the CS-WDA; the broken line is the SPT in $[23,24]$; the full line is the result from the DI-FMT 
The WDA has also been successfully applied to systems with non-planar geometries, like cylindrical pores $[19,25]$ or spherical [26] external potentials, including the test-particle approach to get the pair distribution function $g(r)$ as the (normalized) density profile around a fixed molecule [27]. In those cases, the density distribution still depends on a single variable (the distance to the cylinder axis or to the center of the sphere), so that the computational effort is similar to that of planar walls, although the convolutions to get $\bar{\rho}(r)$ involve a kernel with two variables, $W\left(r, r^{\prime}\right)$, rather than $W\left(z-z^{\prime}\right)$ in (7.42). Some applications to systems with non-planar symmetry, like structured surfaces [28] or slit capillaries [29], have also been explored, with the obvious increase of the computational cost. The present computational capabilities, compared to those in 1985 when the WDA was proposed, certainly play in favor of any DF approach to solve the many interesting problems posed by systems with complex density dependence.

\subsubsection{Weighted Density Approximation for the Hard Sphere Crystal}

The problem of HS crystallization was a main target for the original development of the WDA. The earliest molecular dynamic simulations by Alder and Wainwright [30] in 1960 showed the crystallization of HS at high density. The most accurate estimation for the coexisting mean densities in this first-order phase transition is $\rho_{c}=1.05 \sigma^{-3}$ for the FCC crystal and $\rho_{f}=0.94 \sigma^{-3}$ for the fluid [31, 32, 33] (see also [34] for a comprehensive review of the subject). ${ }^{3}$

Within the scope of this chapter, the HS crystallization was probably the most important problem for the development of DF approximations. The DF approach to this problem sets that the crystal phase should be regarded as a self-structured fluid, for which, even in the absence of any external potential, the minimum of $\Omega[\rho]$ is not achieved by a homogeneous density $\rho(\boldsymbol{r})=\rho_{0}$, but rather by a strongly modulated density $\rho(\boldsymbol{r})$, with the symmetry of the crystal lattice. After some early attempts $[35,36,37]$ to describe crystals with the functional expansion (7.16) around a reference fluid, the first non-perturbative result for the coexisting densities was obtained with the zero-order WDA by Tarazona [16], using a Gaussian parametrization for the density,

$$
\rho(\boldsymbol{r})=\left(\frac{\alpha}{\pi}\right)^{\frac{3}{2}} \sum_{\mathbf{R}} \mathrm{e}^{-\alpha|\mathbf{r}-\mathbf{R}|^{2}},
$$

with normalized peaks at the sites $\mathbf{R}$ of a crystal lattice. This parametrization became standard with the more elaborated CS-WDA and PY-WDA.

The only free parameters to minimize $\Omega[\rho]$ within this restricted functional family are the inverse square Gaussian width $\alpha$ and the crystal lattice parameters. Taking the FCC lattice as the most obvious candidate, we only have

${ }^{3}$ The main branches of the phase diagram of a HS fluid are shown in Fig. 3.5 of Chap. 3 
to fix the lattice length parameter, which is directly given by equating the mean density $\rho_{0}$ to the inverse volume of the unit crystal cell. Therefore, for a fixed mean density, we have to minimize the free energy per unit volume, $f\left(\alpha, \rho_{0}\right) \equiv \rho_{0} \mathcal{F}[\rho] / N$, with respect to the parameter $\alpha$. The results plotted in Fig. 7.4 were obtained with the CS-WDA for three values of $\rho_{0}$, and they show the three qualitatively different regimes which may be found. At low density, the free energy density is minimum at $\alpha=0$, which corresponds to the sum of infinitely flat Gaussians, so that the contributions from all the lattice sites add to a constant homogeneous density $\rho(\boldsymbol{r})=\rho_{0}$; i.e. the parametrization (7.50) contains the uniform fluid phase as the $\alpha=0$ limit, and that is the only stable phase at low density, with a free energy density $f_{f}\left(\rho_{0}\right)$ given by the CS bulk equation of state. For densities slightly above $\rho_{0}=0.9 \sigma^{-3}$, the global minimum of $f\left(\alpha, \rho_{0}\right)$ is still at $\alpha=0$, but there is a local minimum at a large value $\alpha \geq 50 \sigma^{-2}$, which corresponds to a strongly structured density distribution and has to be interpreted as a metastable crystal phase, with free energy $f_{c}\left(\rho_{0}\right)$ per unit volume. Increasing $\rho_{0}$ pushes $f_{c}\left(\rho_{0}\right)$ below $f_{f}\left(\rho_{0}\right)$, so that the crystal phase becomes the most stable phase for $\rho_{0}>1 . \sigma^{-3}$, as shown in Fig. 7.5. The minimization of the grand potential energy per unit volume, $\omega(\mu) \equiv f\left(\rho_{0}\right)-\mu \rho_{0}$, at fixed $\mu$, leads to the usual double tangent construction for the mean densities of the coexisting fluid $\left(\rho_{f} \approx 0.94 \sigma^{-3}\right)$ and crystal $\left(\rho_{c} \approx 1.05 \sigma^{-3}\right)$ phases,

$$
f_{f}^{\prime}\left(\rho_{f}\right)=f_{c}^{\prime}\left(\rho_{c}\right) \equiv \mu_{\text {coex }}
$$

and

$$
\omega_{f}=\omega_{c} \equiv-p_{\text {coex }},
$$

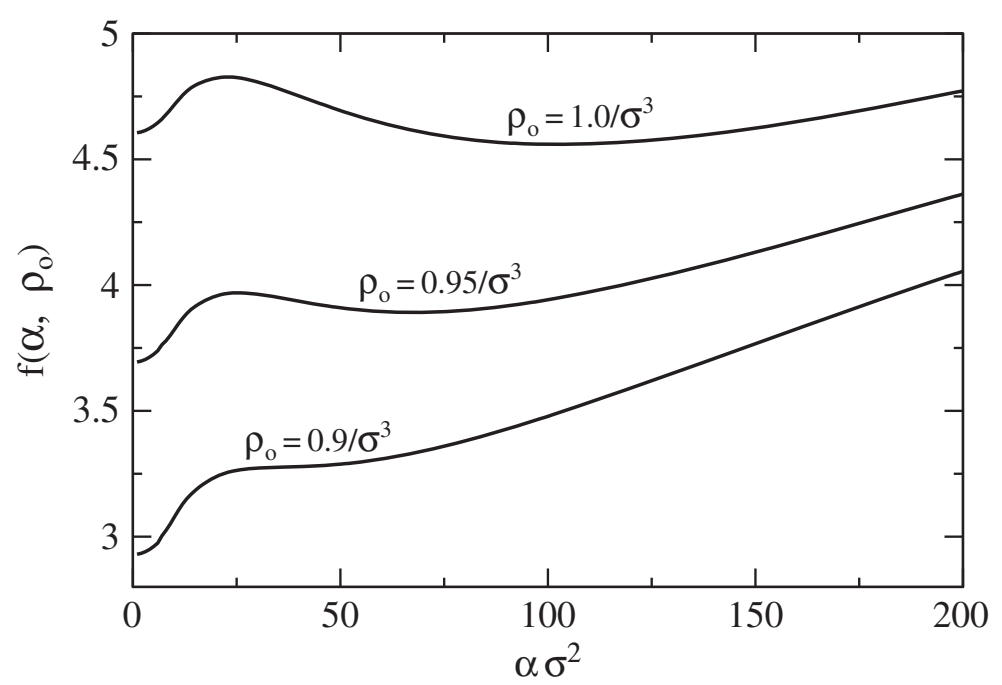

Fig. 7.4. The free energy per unit volume $f\left(\alpha, \rho_{0}\right)$ for the HS crystal, described as a FCC lattice with Gaussian peaks, as a function of the inverse squared width parameter $\alpha$. The minimum at $\alpha=0$ gives the free energy of the fluid phase while at the higher values of the mean density $\rho_{0}$, the crystal phase appears as the (relative or absolute) minimum at $\alpha>50 / \sigma^{2}$ 

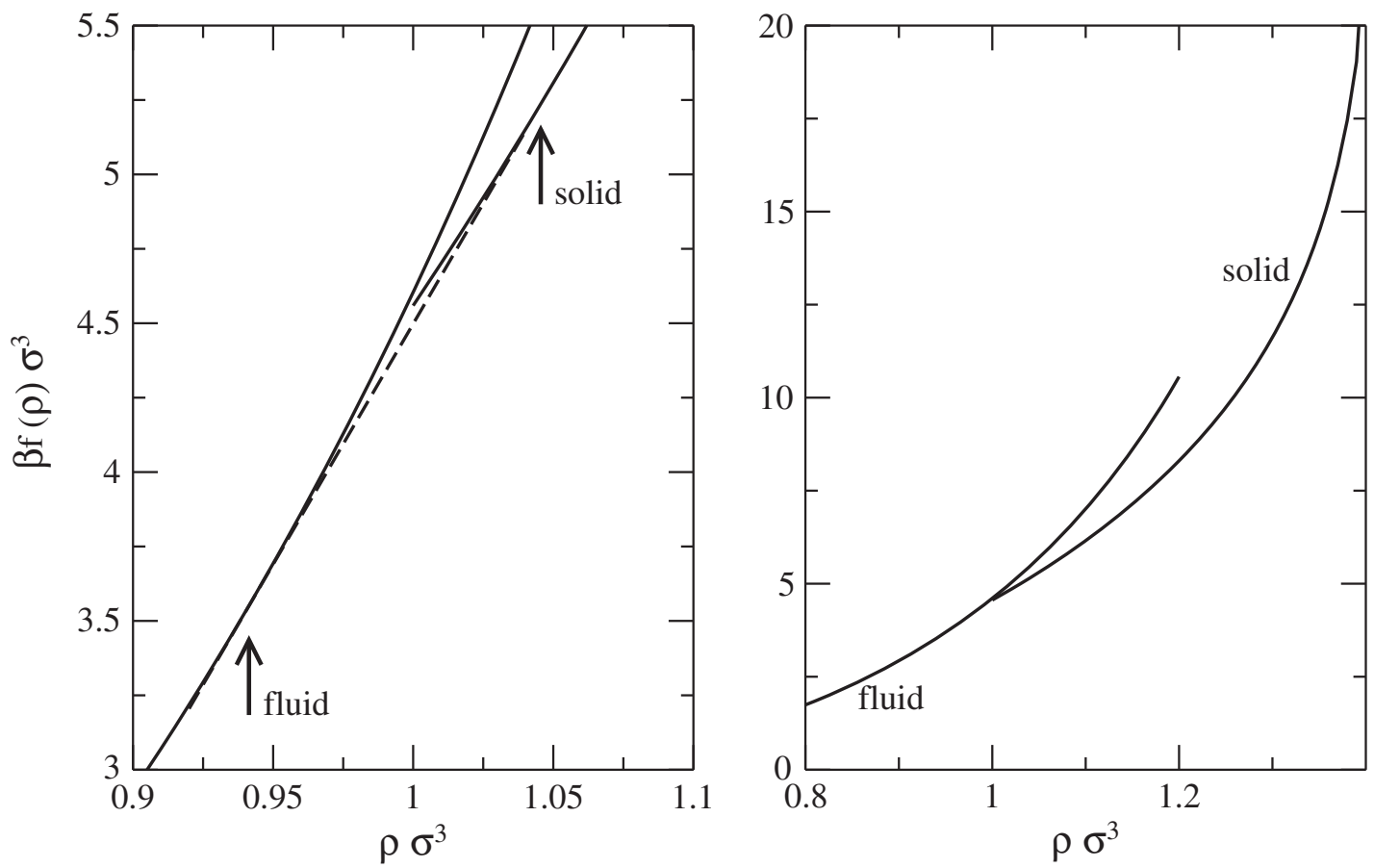

Fig. 7.5. The free energy per unit volume $f\left(\rho_{0}\right)$ for the HS fluid and solid phases, as functions of their mean density, within the CS-WDA with Gaussian peaks on the FCC lattice for the crystal. The left panel gives a detailed view of the transition region, and the broken straight line is the double tangent construction to get the densities of the coexisting phases. The right panel presents the results over the entire range for the solid phase density

with their common pressure, $\beta p_{\text {coex }} \sigma^{3} \approx 11.66$ and chemical potential $\beta \mu_{\text {coex }} \approx$ 16.16.

The numerical evaluation of $f\left(\alpha, \rho_{0}\right)$ within the CS-WDA is simplified with the use of bispherical coordinates for the convolution of a single, normalized, Gaussian peak (set at the origin) with the spherical weight functions centered at a distance $r=|\boldsymbol{r}|$,

$$
\begin{aligned}
\bar{\rho}_{\nu}^{\text {gauss }}(r, \alpha) & \equiv\left(\frac{\alpha}{\pi}\right)^{\frac{3}{2}} \int \mathrm{d} \boldsymbol{r}^{\prime} w_{\nu}\left(\left|\boldsymbol{r}-\boldsymbol{r}^{\prime}\right|\right) \mathrm{e}^{-\alpha r^{\prime 2}} \\
& =\frac{2 \pi}{r}\left(\frac{\alpha}{\pi}\right)^{\frac{3}{2}} \int_{0}^{\infty} \mathrm{d} r^{\prime} r^{\prime} w_{\nu}\left(r^{\prime}\right) \int_{\left|r-r^{\prime}\right|}^{r+r^{\prime}} \mathrm{d} r^{\prime \prime} r^{\prime \prime} e^{-\alpha r^{\prime \prime 2}} \\
& =\frac{1}{r} \sqrt{\frac{\alpha}{\pi}} \int_{0}^{\infty} \mathrm{d} r^{\prime} r^{\prime} w_{\nu}\left(r^{\prime}\right)\left(\mathrm{e}^{-\alpha\left|r-r^{\prime}\right|^{2}}-\mathrm{e}^{-\alpha\left(r+r^{\prime}\right)^{2}}\right) .
\end{aligned}
$$

The $\nu=0$ step weight function gives rise to the analytical expression

$$
\begin{aligned}
\bar{\rho}_{0}^{\text {gauss }}(r, \alpha)= & \frac{3}{8 \pi \sigma^{3}}[\operatorname{erf}(\sqrt{\alpha}(\sigma-r))+\operatorname{erf}(\sqrt{\alpha}(\sigma+r)) \\
& \left.-\frac{\mathrm{e}^{-\alpha(\sigma-r)^{2}}-\mathrm{e}^{-\alpha(\sigma+r)^{2}}}{r \sqrt{\pi \alpha}}\right] .
\end{aligned}
$$


with the error function

$$
\operatorname{erf}(x)=\frac{2}{\sqrt{\pi}} \int_{0}^{x} \mathrm{e}^{-t^{2}} \mathrm{~d} t .
$$

More cumbersome, but still analytical, expressions may be obtained for the empirical $w_{2}(r)$ of the CS-WDA (7.38) and for piecewise polynomial approximants to $w_{1}(r)$. Therefore, the evaluation of the elementary weighted densities $\bar{\rho}_{\nu}(\boldsymbol{r})$ at any position of the crystal is performed via the sum of analytical expressions over the lattice sites $\{\boldsymbol{R}\}$,

$$
\bar{\rho}_{\nu}(\boldsymbol{r}, \alpha)=\sum_{\{\boldsymbol{R}\}} \bar{\rho}_{\nu}^{\text {gauss }}(|\boldsymbol{r}-\boldsymbol{R}|, \alpha) .
$$

For small values of $\alpha$ (near the limit of the uniform fluid), the lattice sum would require many values of $\boldsymbol{R}$, since $\bar{\rho}_{\nu}^{\text {gauss }}(r, \alpha)$ would decay very slowly with $r$. However, the minimum value of $\alpha$ at which the crystal phase appears (even as a metastable phase) is above $50 / \sigma^{2}$, and the contributions to (7.56) decay very fast with $|\boldsymbol{r}-\boldsymbol{R}|$. The numerical calculation is thus very effective on the right-hand side of Fig. 7.4, which is the only relevant part to obtain $f_{c}\left(\rho_{0}\right)$. The evaluation of the full $\bar{\rho}(\boldsymbol{r})$ follows directly from (7.40), and the WDA excess free energy $\Phi([\rho] ; \boldsymbol{r})=\rho(\boldsymbol{r}) \psi(\bar{\rho}(\boldsymbol{r}))$ may be obtained at any point of the space. The integrals of this free energy density and of the ideal gas contribution have to be done over the lattice unit cell, with any quadrature which takes advantage of the point symmetries shared by $\rho(\boldsymbol{r})$ and $\bar{\rho}(\boldsymbol{r})$. Alternatively, we can place a lattice site at the origin and integrate the contribution of that single Gaussian peak,

$$
\beta f\left(\alpha, \rho_{0}\right)=\rho_{0}\left(\frac{\alpha}{\pi}\right)^{\frac{3}{2}} \int \mathrm{d} \boldsymbol{r} \mathrm{e}^{-\alpha r^{2}}[\ln \rho(\boldsymbol{r})-1+\psi(\bar{\rho}(\boldsymbol{r}))],
$$

extended to the whole volume. Notice that both $\rho(\boldsymbol{r})$, in the ideal gas logarithmic term, and $\bar{\rho}(\boldsymbol{r})$, in the excess free energy $\Phi$, have to be evaluated with the full functions, including the contribution of all lattice sites, so that the integrand of (7.57) depends on the direction of $\boldsymbol{r}$. The radial integral may be very effectively done with Gauss-Hermite quadratures, to take advantage of its Gaussian decay, while the double angular integration has to be performed with any standard numerical method. The computation of $f_{c}\left(\alpha, \rho_{0}\right)$ was perfectly feasible twenty years ago, and it is trivially done nowadays.

In Table 7.1 we compare the theoretical and computer simulation results for the coexisting fluid and crystal densities. We also compare the Lindemann ratio of the crystal, which gives the relative mean square displacement of the particles from their lattice positions and which within the Gaussian parametrization (7.50) is given by $L=\left(\rho_{c} / 2\right)^{1 / 3}(3 / \alpha)^{1 / 2}$. The results of the CS-WDA, and to a lesser extent those of the PY-WDA, gave a clear improvement over the existing theories [35, 36, 37], based on the DF 
Table 7.1. The results for the coexisting fluid and crystal densities, in HS diameter units. The Lindemann ratio of the crystal at coexistence is given by $L=\left(\rho_{c} / 2\right)^{1 / 3}(3 / \alpha)^{1 / 2}$ in terms of the Gaussian parametrization of the crystal density distribution

\begin{tabular}{lllll}
\hline & $\rho_{f}$ & $\rho_{c}$ & $\rho_{c}-\rho_{f}$ & $L$ \\
\hline Computer simulations [31] & 0.94 & 1.04 & 0.10 & 0.126 \\
CS-WDA & 0.943 & 1.061 & 0.118 & 0.109 \\
PY-WDA & 0.905 & 1.025 & 0.120 & 0.104 \\
Zero-order WDA [16] & 0.892 & 0.966 & 0.074 & \\
Early perturbative theories [35, 36, 37] & 0.976 & 1.035 & 0.059 & \\
\hline
\end{tabular}

expansion (7.14) around a uniform reference fluid. The density gap between the coexisting phases, which was grossly underestimated by the DF expansion, became slightly overestimated by both WDA versions. The actual values of the coexisting densities are very sensitive to changes in the theory, because as shown in Fig. 7.5, the free energies of the fluid and crystal phases have a nearly tangent intersection. More relevant is the systematic underestimation, by $15-20 \%$ in the Lindemann parameter of the crystal at any given density, which was obtained with any theoretical approach until the advent of the recent FMT versions. $L$ measures the mean squared departures of a HS from its lattice position in the crystal, and a good description of the correlation between the neighbor particles is needed to get the correct amplitudes for the collective excursions of the HS within the crystal. Notice in this respect that the HS crystal is very different from the usual harmonic-crystal model analyzed in solid-state text books; the movement of each HS particle does not follow from the sum of the harmonic normal (i.e. uncorrelated) modes associated to the quadratic expansion of the energy, but it is made of purely free flights between collisions, and all the thermodynamic and elastic properties of the HS crystal follows from purely entropic effects.

The extension of the variational form (7.50) to more flexible shapes was suggested by the observation of a small anisotropy of the particle deviations from their lattice position in computer simulations of the HS crystal. The free minimization over the Fourier space [38] did not change significantly the results of the simplest Gaussian description for the coexisting densities but gave small anisotropies with a sign opposite to that of the simulations. However, the CS-WDA evaluation of the elastic constants of the HS crystal, which also required a deformation of the unit cell density distribution [39], gave good results compared with computer simulations despite the qualitative failure of other theories [40] for the HS crystal.

The most important failure of the WDA description of the HS crystal appears when the density distribution (either within the Gaussian parametrization or the full Fourier space description) is allowed to change the lattice space independently of the mean density. The restriction to have exactly one particle per unit cell, i.e. no vacancies or defect interstitial particles, is a very natural 
simplification of the variational space for $\rho(\boldsymbol{r})$, and it corresponds to what is observed in computer simulations. Nevertheless, we might expect that a good DF approximation should be able to find that unit occupancy of the crystal unit cell from the minimization of $\Omega[\rho]$, but this is not the case for the WDA. The free variation of the normalization in (7.50) increases the mean density of the coexisting crystal by $20 \%$ and destroys the good prediction for the coexistence itself. This problem was not peculiar of the WDA: other DF treatments of the HS crystal, based on refined versions of the functional expansion around a uniform fluid (7.14), shared the same problem. Another qualitative failure of the WDA appears when this DF approximation is used to describe a more open crystal structure, like the BCC lattice. Although that crystal structure (with only eight nearest neighbors) is not expected to be the equilibrium one for HS at any mean density, it is useful to have theoretical results for the HS BCC crystal as a reference system to describe systems with softer repulsions. However, the WDA results for any non-compact crystal structures are unphysical, with the Gaussian parameter $\alpha$ shrinking (instead of growing) when the mean density approaches the complete packing.

These failures of the WDA are not surprising because the full DF approximation is built on the information of the bulk HS fluid. The local correlation structure in the dense HS fluid is not very different from that in an expanded crystal with close-packed structure, with twelve neighbors around each particle, but the local structure in a BCC crystal is completely different from any one likely to appear in a bulk HS fluid, and therefore the WDA has no input to estimate the excess free entropy of such configurations.

\subsubsection{Other Applications and Variants of the WDA}

The generic DF structure of the WDA is not specific for HS; in fact, it was first proposed (under the name of averaged density approximation) for the exchange and correlation energy of the electron liquid [15]. However, its applications to HS systems have probably been the most successful. The WDA concept has also been used to get DF approximations for 2D HD fluids and to study their crystallization [41, 42], although without reaching the specific features of 2D crystallization. The role of dislocations and disclinations of the perfect crystal phase and the possible presence of an intermediate hexatic phase would be very difficult targets for any DF treatment of the crystal, described as a self-structured fluid at the level of the one-particle density distribution, and they are certainly beyond the WDA. Whether or not the effects of those topological defects may be included with a more accurate representation of $\mathcal{F}_{\text {ex }}[\boldsymbol{\rho}]$ is still an open question. A further reduction of the dimension leads to the exact results for 1D HR (7.20) and (7.21), which includes the disruption of the long-ranged $1 \mathrm{D}$ order by point defects, so that there is no phase transition and the fluid phase is stable at any mean density $\rho_{0} \sigma<1$. The fact that a relatively simple analytic form of $\mathcal{F}_{\text {ex }}[\boldsymbol{\rho}]$ is able to describe, at the $\rho(x)$ level, an effect arising from global correlations extended to the 
whole 1D system gives hope to the future DF description of the complex 2D crystallization. On the opposite sense, the accuracy of the WDA when applied to systems of hyper-spheres, in $D>3$ dimensions, should increase with $D$, because its DF structure contains the basic elements of the low-density expansion (7.16), and the maximum density allowed by the packing constraints decreases as $D$ increases.

Some early attempts were made to apply the WDA to simple fluid models, with both attractive and repulsive regions of their interatomic potential, but it was soon clear that the non-local aspects associated to the entropy loss by the core repulsions cannot be directly added to those associated to the energy of the attractive interactions. The best DF approximations for these systems are based on the separated treatment of a reference HS system and the MFA [2, 19], or similar DF schemes, for the description of the attractive interactions [43]. For purely repulsive, but soft, pair interaction potentials, the WDA has been applied with moderate success, allowing for temperature-dependent weight functions [44, 45, 46]. However, the quality of the approximation rapidly decreases as the repulsive potential $u(r)$ becomes softer, down to a rather discrete accuracy for the soft repulsion of the onecomponent plasma.

Several variants of the WDA scheme for HS have been developed and explored, among them that of Meister and Kroll [2, 47, 48], who explored a variational method to determine $\bar{\rho}(\boldsymbol{r}, \rho)$ within a DF expansion from a WDA scheme (see relevant comments in [2]). Other variants of the WDA pointed to a simplification of its practical use for the HS crystal, for its own sake or as reference system for the solid phase of other simple fluid models. The modified weighted density approximation (MWDA) of Denton and Ashcroft [49, 50, 51] takes a constant weighted density over the entire system, rather than being a function of the local position. That provides a strong simplification in the numerical use of the theory, which becomes equivalent to the effective liquid approximation (ELA) and its variants (GELA, MELA) [52, 53], in which the crystal phase is described in a perturbative scheme around a uniform fluid phase. The obvious drawback of these approaches, with respect to any DF approximation, is that they cannot be applied to generic inhomogeneous distributions $\rho(\mathbf{r})$.

\subsection{The Fundamental Measure Theory}

In 1989, a new DF approximation for the HS fluid was proposed by Rosenfeld [13] under the name of FMT, and it represented a breaking point in the theory of inhomogeneous hard body systems. That original version will be referred here as the oFMT to distinguish it from its subsequent variants, proposed and tested by different authors. By the end of the century, it was clear that the generic FMT scheme is qualitatively superior to any WDA although it requires a much more careful design of the functional form and it also has some 
technical complexities, like the use of vector and tensor weighting functions, which have probably scared some potential users. This section gives a brief account of the main concepts of the FMT and the most advanced version, based on the idea of dimensional interpolation. We present detailed recipes for the practical application of the FMT to the most usual symmetries and comments on the FMT results for the most important problems.

\subsubsection{Rosenfeld's Original Fundamental Measure Theory}

Fundamental and subtle clues led Rosenfeld to the original development of the FMT $[12,13]$, which was directly set as a DF theory for HS mixtures, as we review in Sect. 7.8. Here, we present first the mono-component case, with some changes in the original notation, to give a simpler account of both the original FMT version and the most recent developments. The first point to set up the FMT is to include the packing fraction as an essential non-local measure for the free energy of HS. The best approximations for the equation of state and correlation structure of the HS fluid cast the density dependence in terms of the dimensionless packing fraction $\eta=\pi \rho \sigma^{3} / 6$, i.e. the ratio of the volume occupied by the HS with respect to the total system volume. The natural extension to inhomogeneous systems is the local packing fraction,

$$
\eta(\boldsymbol{r}) \equiv \int \mathrm{d} \boldsymbol{r}^{\prime} \rho\left(\boldsymbol{r}+\boldsymbol{r}^{\prime}\right) \Theta\left(\frac{\sigma}{2}-\left|\boldsymbol{r}^{\prime}\right|\right)
$$

which is a crucial piece of the exact free energy DF for 1D HR (7.21) and (7.22). Notice that $\eta(\boldsymbol{r})$ has the appealing interpretation of being the probability that for random molecular configurations over the equilibrium statistical ensemble, the point $\boldsymbol{r}$ happens to be inside a HS core. A density distribution producing anywhere a value $\eta(\boldsymbol{r})>1$ is therefore forbidden for the HS fluid, so that the use of this non-local measure gives a simple tool to locate the DF borders of the accessible $\rho(\boldsymbol{r})$, much more accurately than the lack of solutions for $\bar{\rho}(\boldsymbol{r})$ in the WDA, although still not in a complete form. ${ }^{4}$

Notice that (7.58) is very different from the zero-order weighted function in the WDA (7.32), since $w_{0}(r)$ is a (normalized) Mayer function, i.e. a radial step function with radius equal to the HS diameter $\sigma$ while the radius of the step function defining $\eta(\boldsymbol{r})$ is the molecular radius $R \equiv \sigma / 2$. A main concept of the FMT is precisely that the fundamental measure to describe the nonlocal dependence of $\Phi([\rho] ; \boldsymbol{r})$ is the shape of a single molecule rather than the excluded volume between two molecules; therefore the free energy DF is

\footnotetext{
${ }^{4}$ The restriction to $\eta(\boldsymbol{r}) \leq 1$ is a necessary but not sufficient condition to have a density distribution $\rho(\boldsymbol{r})$ compatible with the HS core repulsion. Obviously, $\eta(\boldsymbol{r})$ cannot take, over extensive regions, values larger than the close packing of spheres $\eta=0.74$ although it may approach 1 over small regions of the size of a sphere. See the discussion in Sect. 7.5.2 and in [54] for the smallest cases in which the accessible DF boundary moves below $\eta=1$.
} 
represented as a function of $\eta(\boldsymbol{r})$ and other weighted densities, which are all the convolutions of $\rho(\boldsymbol{r})$ within the range of the HS radius.

The exact free energy for 1D HR given in (7.21) has precisely the form

$$
\Phi^{(1 \mathrm{D})}([\rho] ; x)=\phi_{1}(\eta(x), n(x)) \equiv-n(x) \ln [1-\eta(x)]
$$

with the local packing fraction (7.22) and the combination $n(x) \equiv[\rho(x+R)+$ $\rho(x-R)] / 2$, which may be regarded as the $1 \mathrm{D}$ version of a generic surface weighted density,

$$
n(\boldsymbol{r}) \equiv \int \mathrm{d} \boldsymbol{r}^{\prime} \rho\left(\boldsymbol{r}+\boldsymbol{r}^{\prime}\right) w\left(\boldsymbol{r}^{\prime}\right)
$$

with the normalized molecular surface weight function,

$$
w(\boldsymbol{r}) \equiv \frac{\delta(|\boldsymbol{r}|-R)}{s_{\mathrm{D}}}
$$

$s_{\mathrm{D}}$ being the total molecular surface in $D$ dimensions $\left(s_{1}=2, s_{2}=2 \pi R\right.$, $\left.s_{3}=4 \pi R^{2}\right)$. Notice that we are not following Rosenfeld's original notation: the scalar density $n(\boldsymbol{r})$ defined in (7.60) and (7.61) equals the homogeneous density for any distribution $\rho(\boldsymbol{r})=\rho_{0}$.

There is an interesting difference between the two alternative forms (7.20) and (7.21) which can be used to describe the exact excess free energy of 1D HR systems. In the first one, $\Phi^{(1 \mathrm{D})}([\rho] ; x)$ is explicitly proportional to the local density $\rho(x)$, so that (like in the WDA) the free energy excess is locally assigned to the position of the particle centers, with a value per particle which depends on the sampling of the density up to distances $\sigma$ around the point $\boldsymbol{r}$.

In contrast, the expression $(7.21)$ for $\Phi^{(1 \mathrm{D})}([\rho] ; x)$ is not proportional to $\rho(x)$, and this functional form may assign a local density of excess free energy to regions with $\rho(x)=0$ but $\eta(x)>0$. The proportionality with $n(x)$ in (7.59) has to be interpreted as the assignment of the free energy excess to the positions of the molecular surface, i.e. of the two ends of the 1D HR. Obviously, there is nothing wrong in having different exact expressions for $\Phi^{(1 \mathrm{D})}[\rho ; x]$, as far as they all integrate to the same $\mathcal{F}_{\text {ex }}[\rho]$ for any density distribution. The no-overlap restriction imposed by the hard cores produces a global entropy decrease, which may be locally assigned in different ways, to get the free energy density $\Phi^{(1 \mathrm{D})}([\rho] ; x)$.

For $1 \mathrm{D} \mathrm{HR}$, the exact $\rho(x) \rightarrow 0$ limit of (minus) the direct correlation function (7.17) is recovered from (7.21) as

$$
f(x) \equiv \Theta(\sigma-|x|)=\frac{1}{2} \int \mathrm{d} x^{\prime} \delta\left(\frac{\sigma}{2}-\left|x+x^{\prime}\right|\right) \Theta\left(\frac{\sigma}{2}-\left|x^{\prime}\right|\right)
$$

where the Mayer function range, $-\sigma \leq x \leq \sigma$, arises from the convolution of the two fundamental measures, each with half that range. The $3 \mathrm{D}$ geometry of the HS makes it impossible to represent the spherical step of the Mayer function, $f(\boldsymbol{r})=\Theta(\sigma-|\boldsymbol{r}|)$, in terms of the half-ranged $(R=\sigma / 2)$ step 
$w_{0}(\boldsymbol{r})=\Theta(R-|\boldsymbol{r}|)$ in (7.58) and the spherical shell (7.61). That explains the relatively poor performance of the direct extension of the $1 \mathrm{D}$ free energy DF form to describe 3D HS, attempted by Robledo et al. [10, 11]. To solve this problem, Rosenfeld made the crucial proposal to enlarge the set of fundamental measure functions with a vector weight,

$$
\boldsymbol{w}(\boldsymbol{r}) \equiv \frac{\boldsymbol{r}}{R} \frac{\delta(R-|\boldsymbol{r}|)}{s_{\mathrm{D}}},
$$

to define a vector-weighted density,

$$
\boldsymbol{v}(\boldsymbol{r}) \equiv \int \mathrm{d} \boldsymbol{r}^{\prime} \rho\left(\boldsymbol{r}+\boldsymbol{r}^{\prime}\right) \boldsymbol{w}\left(\boldsymbol{r}^{\prime}\right)
$$

The packing fraction (7.58), the scalar-weighted density (7.60) and the vector weighted density (7.64) are used as the three local variables to describe $\Phi_{\text {FMT }}([\rho] ; \boldsymbol{r})=\phi(\eta(\boldsymbol{r}), n(\boldsymbol{r}), \boldsymbol{v}(\boldsymbol{r}))$. The lowest (quadratic) order of this function must depend on its three variables in the form $\phi(\eta, n, \boldsymbol{v})=$ $\eta n+4 \pi R^{3}\left(n^{2}-\boldsymbol{v} \cdot \boldsymbol{v}\right)+\mathcal{O}^{3}(\eta, n, \boldsymbol{v})$. This is the unique combination which recovers the exact low-density expansion of the direct correlation function (7.17), since the unique geometrical construction to extend (7.62) to $3 \mathrm{D}$ is

$$
\begin{aligned}
f(r) \equiv \Theta(\sigma-|\boldsymbol{r}|)= & \frac{1}{2 \pi R^{2}} \int \mathrm{d} \boldsymbol{r}^{\prime} \delta\left(R-\left|\boldsymbol{r}^{\prime}\right|\right)\left[\Theta\left(R-\left|\boldsymbol{r}+\boldsymbol{r}^{\prime}\right|\right)\right. \\
& \left.+R \delta\left(R-\left|\boldsymbol{r}+\boldsymbol{r}^{\prime}\right|\right)\left(1-\frac{\boldsymbol{r}^{\prime} \cdot\left(\boldsymbol{r}+\boldsymbol{r}^{\prime}\right)}{R^{2}}\right)\right] .
\end{aligned}
$$

The final clue followed by Rosenfeld was that the structure of the spherical shell weight functions, $w(\boldsymbol{r})$ and $\boldsymbol{w}(\boldsymbol{r})$, is directly related to the derivatives of $\Theta(R-|\boldsymbol{r}|)$ with respect to the molecular size, suggesting a connection with the scaled particle theory (SPT) for the thermodynamic properties of hard bodies $[23,24,55]$. This theory reproduces the exact equation of state in $1 \mathrm{D}$ and the PY compressibility result for 3D HS.

The search for a function $\phi(\eta, n, \boldsymbol{v})$ with the exact low-density limit and reproducing the PY direct correlation function led to the oFMT form

$$
\Phi_{\mathrm{oFMT}}([\rho] ; \boldsymbol{r})=\sum_{i=1}^{3} \phi_{i}^{(3 \mathrm{D})}(\eta(\boldsymbol{r}), n(\boldsymbol{r}), \boldsymbol{v}(\boldsymbol{r})),
$$

with the first term $\phi_{1}^{(3 \mathrm{D})}(\eta, n)=-n \ln (1-\eta)$ identical to the full 1D result (7.59), and two more terms given by

$$
\phi_{2}^{(3 \mathrm{D})}(\eta, n, \boldsymbol{v})=4 \pi R^{3} \frac{n^{2}-\boldsymbol{v} \cdot \boldsymbol{v}}{1-\eta}
$$

and

$$
\phi_{3}^{(3 \mathrm{D})}(\eta, n, \boldsymbol{v})=8 \pi^{2} R^{6} n \frac{n^{2} / 3-\boldsymbol{v} \cdot \boldsymbol{v}}{(1-\eta)^{2}}
$$


The results of this DF approximation were a puzzling mixture of successes and failures: the density profiles for HS against a hard wall are clearly better than those obtained with the PY-WDA version, particularly in reproducing the strong oscillatory structure for a high-density bulk fluid, as will be shown in Fig. 7.9. The equation of state plugged into the CS-WDA provides a better value of the contact density, but it still does not match the excellent description of $\rho(z)$ given by the oFMT for the interlayer spacing, reflecting a sharper representation of the correlation structure in inhomogeneous systems. Also, the second functional derivative of the oFMT excess free energy is restricted to the PY range, $\left|\boldsymbol{r}-\boldsymbol{r}^{\prime}\right| \leq \sigma \equiv 2 R$, not only for bulk liquids but for any density distribution. In contrast, the PY-WDA built on the same results for the bulk liquid direct correlation has a second functional derivative which goes beyond that range for inhomogenous density distributions, since the precise tailoring of the oscillating tail in $w(r, \rho)$ may only restrict the range for the homogeneous fluid direct correlation. Another very appealing feature of the oFMT was its natural extension to HS mixtures, in contrast with the conceptual difficulties of the WDA for that task. We will elaborate more on this point later in Sect. 7.6.

However, the oFMT has some qualitative pitfalls related to the use of deltafunction shells in $w(\boldsymbol{r})$ and $\boldsymbol{w}(\boldsymbol{r})$. The overlap of these shells produces strong divergences, which have to be tamed by the careful choice of the combinations (7.67), (7.68) of $n(\boldsymbol{r})$ and $\boldsymbol{v}(\boldsymbol{r})$. It is a generic feature of the FMT that slight variants of a good approximation may be qualitatively wrong, in contrast with the mild dependence of the WDA on the details of each particular version. Still, the combination of $n$ and $\boldsymbol{v}$ used in (7.68) can only avoid the unphysical divergences of $\Phi([\rho] ; \boldsymbol{r})$ for homogeneous systems, and its application to highly inhomogeneous systems, like the DF description of the HS crystal, leads to a dramatic failure: as the Gaussian peaks shrink to delta functions, the free energy of the crystal phase decreases without bound. It took ten years to develop new versions of the FMT with this problem corrected and able to provide an overall improvement over the WDA in all aspects, as we review below.

This notwithstanding, the oFMT remains an excellent, and fairly used, DF choice to solve problems of highly packed HS (and their mixtures) with planar geometry, where the homogeneity over the transverse directions is enough to avoid the divergences of the radial shells overlap. The practical implementation of those applications is discussed below, together with that of the more recent FMT versions. The vector weighted density, $\boldsymbol{v}(\boldsymbol{r})$, gave the oFMT a less intuitive character than the WDA, which has probably restrained some potential users. The FMT version proposed by Kierlik and Rosinberg [56, 57] substituted $\boldsymbol{v}(\boldsymbol{r})$ with the gradient of $\eta(\boldsymbol{r})$. The resulting DF is perhaps more intuitive, but with little (or none) advantage for the practical use of the theory. In fact, the same authors proved that both versions of the FMT (with and without vector density) are identical because they both yield the same free energy DF [58]. 


\subsubsection{Dimensional Crossover and the Cavity Theory}

The concept of dimensional crossover is a strong test for DF approximations which was first applied [19] to the CS-WDA for HS. The idea is that any DF approximation for the $3 \mathrm{D}$ excess free energy of $\mathrm{HS}, \mathcal{F}_{\text {ex }}[\rho]$, contains also predictions for any 2D distribution of $\mathrm{HD}, \rho_{2 \mathrm{D}}(x, y)$, because the latter should be fully equivalent to a $3 \mathrm{D}$ distribution with a delta function along the $Z$ axis: $\rho_{3 \mathrm{D}}(x, y, z)=\rho_{2 \mathrm{D}}(x, y) \delta(z)$; see Fig. 7.6. The extremely strong inhomogeneity of that $3 \mathrm{D}$ density distribution provides a severe test for any DF approximation to $\mathcal{F}_{\text {ex }}[\rho]$, and the WDA showed a rather limited success in this respect. The dimensional crossover may be extended to represent 1D distributions of $\mathrm{HR}$ as $3 \mathrm{D}$ distributions, with $\rho_{3 \mathrm{D}}(x, y, z)=\rho_{1 \mathrm{D}}(x) \delta(y) \delta(z)$, and the equivalent reduction from $2 \mathrm{D}$ to $1 \mathrm{D}$ can also be defined. The original FMT version for $3 \mathrm{D}$ HS and its 2D counterpart were subjected to those tests [59] in order to extract the predictions for the excess free energy of uniform $2 \mathrm{D}$ and $1 \mathrm{D}$ systems from their higher dimensional representations. The results were again a mixture of success and pitfalls: the DF reduction of the oFMT from 3D to 2D is very accurate; however, the reduction from $3 \mathrm{D}$ to $1 \mathrm{D}$ was a complete failure, since the third term $\phi_{3}^{(3 \mathrm{D})}$ in (7.66) diverges. Nevertheless, it was pointed out that if that term were eliminated, the sum $\phi_{1}^{(3 \mathrm{D})}+\phi_{2}^{(3 \mathrm{D})}$ would give the exact result for $\Phi^{(1 \mathrm{D})}$.

The DF dimensional crossover was pushed forward with the concept of the zero-dimensional (0D) limit, leading to the development of the cavity
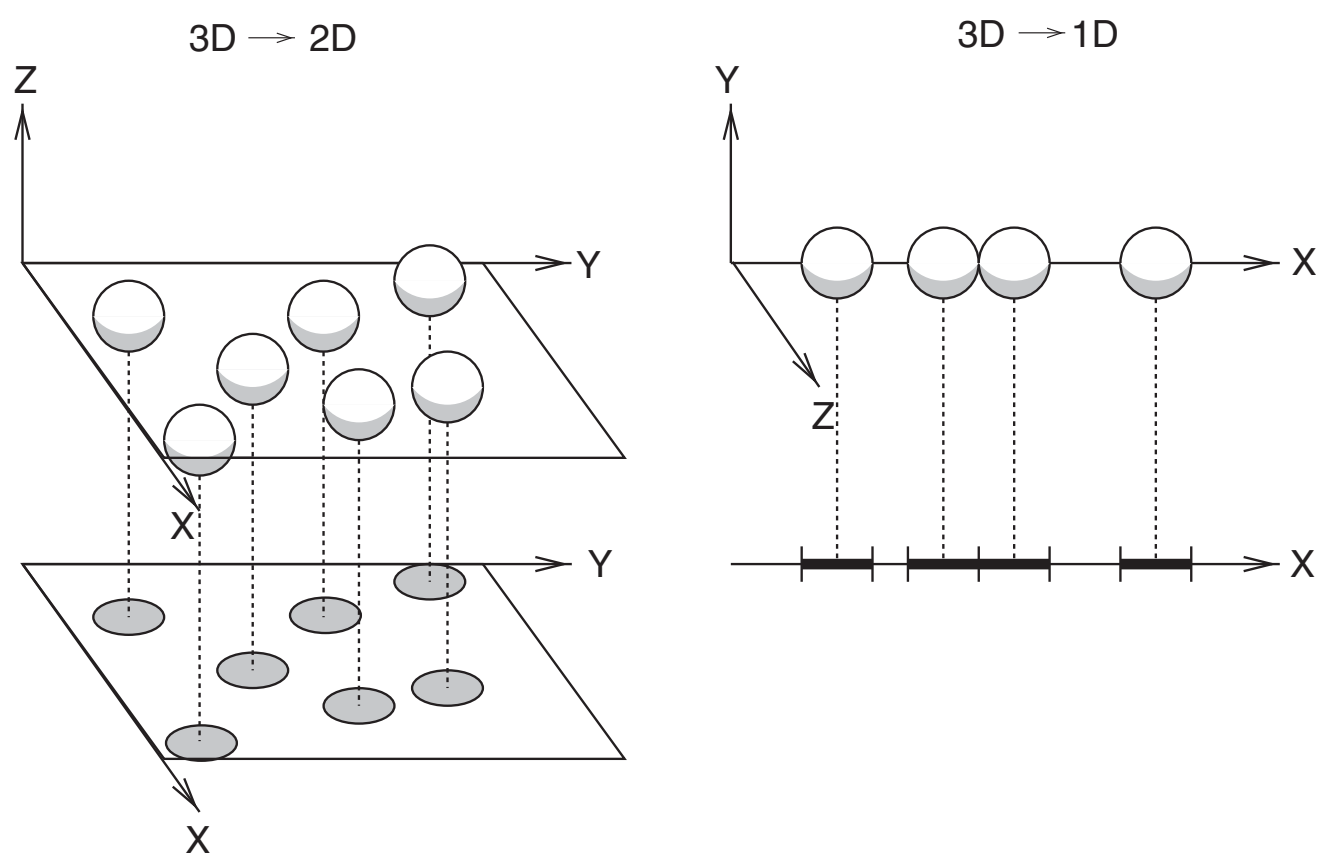

Fig. 7.6. Sketches of dimensional crossover within the DF formalism. On the left, a $3 \mathrm{D}$ distribution with the hard sphere centers located on the $z=0$ plane $\rho_{3 \mathrm{D}}(x, y, z)=$ $\delta(z) \rho_{2 \mathrm{D}}(x, y)$ is fully equivalent to a $2 \mathrm{D}$ distribution of hard disks $\rho_{3 \mathrm{D}}(x, y, z)=$ $\delta(y) \delta(z) \rho_{1 \mathrm{D}}(x)$. On the right, a $3 \mathrm{D}$ distribution with all the HS centers along the $X$ axis is fully equivalent to a system of $1 \mathrm{D}$ hard rods 
theory by Tarazona and Rosenfeld [54], which gave a fresh view to the FMT. Consider a small cavity which cannot hold more than one HS and which is connected to a particle reservoir at chemical potential $\mu$. We use $\eta_{0} \leq 1$ as the mean number of particles in the cavity, i.e. the probability that the cavity is occupied. Unlike the $2 \mathrm{D}$ and $1 \mathrm{D}$ cases, the exact $0 \mathrm{D}$ limit does not only cover the strict reduction of the geometrical dimension, $\rho(\boldsymbol{r})=\eta_{0} \delta(x) \delta(y) \delta(z)$, but also a wide range of smoother $3 \mathrm{D}$ density distributions, with the only restriction that the distance between any two points with $\rho(\boldsymbol{r}) \neq 0$ is less than a HS diameter $\sigma=2 R$. The exact excess free energy $\mathcal{F}_{\text {ex }}[\rho]$ may be obtained for any density distribution $\rho(\boldsymbol{r})$ allowed within the cavity, because its grand partition function (7.3) takes exactly the form $\Xi_{0 \mathrm{D}}=1+Z_{1} \exp (\beta \mu)$, with the two first terms of the ideal gas expression (7.5) and the total integral of the density distribution, i.e. the total occupancy of the cavity, is exactly $\eta_{0}=Z_{1} \exp (\beta \mu) / \Xi_{0 \mathrm{D}}$. Using this expression, eliminate the dependence of $\Xi_{\mathrm{OD}}$ with the chemical potential and the confining external potential in $Z_{1}$. The exact generic form of the $0 \mathrm{D}$ excess free energy is obtained as $\beta \mathcal{F}_{\text {ex }}=$ $\phi_{0}\left(\eta_{0}\right) \equiv\left(1-\eta_{0}\right) \ln \left(1-\eta_{0}\right)+\eta_{0}$, which depends on $\rho(\boldsymbol{r})$ only through its total integral $\eta_{0}$, independently of the shape of the density distribution within the cavity.

That 0D excess free energy has the low-density expansion $\phi_{0}\left(\eta_{0}\right)=$ $\eta_{0}^{2} / 2+\eta_{0}^{3} / 6+\cdots$, which could have been extracted from (7.16), since the 0D character of the cavity implies that $f\left(r_{i j}\right)=1$ for any pair of points with $\rho\left(\boldsymbol{r}_{i}\right)>0$ and $\rho\left(\boldsymbol{r}_{j}\right)>0$. In the opposite limit, for cavities which are nearly always occupied, the free energy excess goes to $\phi_{0}(1)=1$ with diverging slope, as it corresponds to the physical condition that the full occupancy of the cavity implies the lack of empty states in the total partition function, which can only be achieved with a divergently high chemical potential in the reservoir.

The requirement that any $\rho(\boldsymbol{r})$ within a $0 \mathrm{D}$ cavity has an excess free energy $\phi_{0}\left(\eta_{0}\right)$ is a very severe condition which imposes strong restrictions on the non-local dependence of $\mathcal{F}_{\text {ex }}[\rho]$. The cavity theory [54] shows that a full $\mathcal{F}_{\text {ex }}[\rho]$ for $3 \mathrm{D}$ HS (and also for 2D HD) may be built using only the requirement that the exact 0D limit is recovered over a partial set of cavity shapes. That free energy DF has the essential elements of the FMT: the use of $\eta(\boldsymbol{r})$ and density convolutions with spherical delta-function shells or radius $R$. The geometrical combinations of those convolutions are fixed in such way that the divergences produced when they overlap are fully eliminated. Within this 0D FMT approach, the thermodynamics of the uniform bulk HS fluid is a particular output of the DF approximation rather than an input to be used in the construction of $\mathcal{F}_{\text {ex }}[\rho]$. The 0D FMT excess free energy density for the bulk HS fluid is directly given in terms of the successive derivatives of the $0 \mathrm{D}$ free energy excess, $\phi_{0}(\eta)$, with respect to $\eta$ : $\phi_{0}^{\prime}(\eta)=-\ln (1-\eta), \phi_{0}^{\prime \prime}(\eta)=1 /(1-\eta)$ and $\phi_{0}^{\prime \prime \prime}(\eta)=1 /(1-\eta)^{2}$, something that already appears in the result of the SPT and that was directly plugged into the oFMT (7.66) and (7.68). Rosenfeld had coined the term ideal liquid [60] to refer to the (unreachable) 
limit $\eta \rightarrow 1$ of the HS fluid, since the inverse powers of $1-\eta$ appear in any good representation of the equation of state for the HS bulk fluid despite the much lower geometrical close packing of spheres (with maximum mean value $\left.\eta_{\max }=0.740\right)$. Within the cavity theory, that ideal liquid limit of the bulk fluid appears naturally as a reminiscence of the physical singularity in the 0D excess free energy at $\eta_{0}=1$.

The DF extracted from the 0D limit confirms that the FMT elements are the natural building blocks of $\mathcal{F}_{\text {ex }}[\rho]$, but it also unveils the limitations of any DF approximation based on one-center convolutions. The structure of the 0D FMT free energy DF follows closely that set in the oFMT: the free energy density for isotropic hard core bodies in $D$ dimensions has precisely $\nu=1, \ldots, D$ terms, with the dependence on the local packing fraction given by the $\nu$ th-derivative of $\phi_{0}(\eta)$ and with an order $\nu$ convolution of the density with spherical delta-function shells, plus geometrical factors which eliminate all the spurious divergences. The first-order term in any dimension has the exact form of the $1 \mathrm{D}$ free energy, $\phi_{1}^{(1 \mathrm{D})}(\eta, n)=n \phi_{0}^{\prime}(\eta)$, identical to Rosenfeld's choice in (7.66). Also the second term for the 3D case becomes exactly that in the oFMT form, $\phi_{2}^{(3 \mathrm{D})}(\eta, n, \boldsymbol{v})=4 \pi R^{3}\left(n^{2}-\boldsymbol{v} \cdot \boldsymbol{v}\right) \phi_{0}^{\prime \prime}(\eta)$. However, the third term for HS (and the second term for 2D HD) cannot be separated in terms of one-center convolutions, like $n(\boldsymbol{r})$ and $\boldsymbol{v}(\boldsymbol{r})$. The appearance of non-separable convolutions was already discussed in the comparison between the WDA and the exact density expansion (7.16), and there is a strong practical bias toward one-center DF forms. Moreover, even if the non-separable kernels were accepted in $\phi_{3}^{(3 \mathrm{D})}$, the resulting DF approximations still leave out a class of 0D cavities, which were called the lost cases of the FMT [54], for which the local packing fraction $\eta(\boldsymbol{r})$ does not reach anywhere the total integral $\eta_{0}$ of the density distribution $\rho(\boldsymbol{r})$. The simplest example of a lost-case cavity is made of three point-like subcavities, at the vertex of an equilateral triangle, separated by a distance between $\sqrt{3} R$ and $2 R$, so that any two of them cannot be occupied at the same time, but there is no point closer than $R$ to the three subcavities, where $\eta(\boldsymbol{r})$ would be the sum of the three partial occupancies. Forbidden density distributions, with $1<\eta_{0} \leq 3 / 2$, are compatible with the restriction $\eta(\boldsymbol{r}) \leq 1$, and they cannot be excluded within the FMT scheme of one-center convolutions.

The importance of these lost cases increases with the dimension of the supporting space. They are absent in cavities with 1D support, and the exact $\Phi^{(1 \mathrm{D})}([\rho] ; \boldsymbol{r})$ of Percus may be built from the 0D FMT. In 2D, there are some 0D lost cases, and the 0D FMT does not lead to the exact result, but still it leads to the (fairly accurate) SPT for the equation of state of homogeneous systems of $\mathrm{HD}$. In 3D, the increasing role of the lost cases is reflected in a rather poor approximation for the bulk HS equation of state given by the 0D FMT free energy DF. The compromise between a good dimensional reduction, a good description of the HS bulk fluid and the practical handling of the DF approximation leads to the recent DF approach presented in the next section. 


\subsubsection{The Dimensional Interpolation Fundamental Measure Theory}

The dimensional interpolation (DI) version of the FMT for HS was introduced [61] as a practical approximation which balances the advantages of the previous proposals. It is built to recover the PY direct correlation function (and hence the PY-compressibility equation of state) for bulk homogeneous HS fluids, as in the original FMT version of Rosenfeld, but using the 0D FMT basic elements to systematically eliminate the spurious divergences in the $1 \mathrm{D}$ and $0 \mathrm{D}$ reductions. As the original FMT version, the DI-FMT excess free energy $\Phi^{(3 \mathrm{D})}([\rho] ; \boldsymbol{r})$ has three additive terms;

$$
\Phi^{(3 \mathrm{D})}([\rho] ; \boldsymbol{r})=\sum_{\nu=1}^{3} \phi_{\nu}^{(3 \mathrm{D})}(\eta(\boldsymbol{r}), n(\boldsymbol{r}), \ldots),
$$

and the first two have exactly the same form proposed by Rosenfeld, in terms of the local packing fraction, the scalar and the vector density convolutions, namely

$$
\phi_{1}^{(3 \mathrm{D})}(\eta, n)=-n \ln (1-\eta), \quad \phi_{2}^{(3 \mathrm{D})}(\eta, n, \boldsymbol{v})=4 \pi R^{3} \frac{n^{2}-\boldsymbol{v} \cdot \boldsymbol{v}}{1-\eta} .
$$

The third term requires a new tensor density, $\mathcal{T}(\boldsymbol{r})$, with cartesian components $(\alpha, \beta=x, y, z)$ defined by the convolution

$$
\mathcal{T}_{\alpha \beta}(\boldsymbol{r}) \equiv \int \mathrm{d} \boldsymbol{r}^{\prime} \rho\left(\boldsymbol{r}+\boldsymbol{r}^{\prime}\right) \frac{r_{\alpha}^{\prime} r_{\beta}^{\prime}}{R^{2}} \frac{\delta\left(R-\left|\boldsymbol{r}^{\prime}\right|\right)}{4 \pi R^{2}}
$$

There is a unique combination of this tensor with $n(\boldsymbol{r}), \boldsymbol{v}(\boldsymbol{r})$ and $\eta(\boldsymbol{r})$ which added to $\Phi_{1}^{(3 \mathrm{D})}+\Phi_{2}^{(3 \mathrm{D})}$ recovers the bulk PY direct correlation for $\rho(\boldsymbol{r})=\rho_{0}$ and the exact free energy for the strict 0D limit, $\rho(\boldsymbol{r})=\eta_{0} \delta(\boldsymbol{r})$. That combination defines the DI-FMT version with

$$
\Phi_{3}^{(3 \mathrm{D})}(\eta, n, \boldsymbol{v}, \mathcal{T})=12 \pi^{2} R^{6} \frac{\boldsymbol{v} \cdot \mathcal{T} \cdot \boldsymbol{v}-n \boldsymbol{v} \cdot \boldsymbol{v}-\operatorname{Tr}\left[\mathcal{T}^{3}\right]+n \operatorname{Tr}\left[\mathcal{T}^{2}\right]}{(1-\eta)^{2}},
$$

in terms of the rotational invariants formed by the index contractions of $\mathcal{T}$ and $\boldsymbol{v}$, as well as the traces of $\mathcal{T}, \mathcal{T}^{2}$ and $\mathcal{T}^{3}$. Notice that the scalar density is precisely $n(\boldsymbol{r})=\operatorname{Tr}[\mathcal{T}]$.

The original Rosenfeld's proposal (7.68) for $\Phi_{3}^{(3 \mathrm{D})}(\eta, n, \boldsymbol{v})$ may be recovered from (7.72) with the extra assumption that the tensor $\mathcal{T}$ is approximated as $n / 3$ times the unit $3 \mathrm{D}$ tensor, i.e. neglecting its anisotropy but keeping its trace. That explains the good results of the oFMT for planar density profiles, $\rho(\boldsymbol{r})=\rho(z)$, since the homogeneity over the $X Y$ directions renders the anisotropy of $\mathcal{T}(\boldsymbol{r})$ nearly irrelevant because, by symmetry, this tensor is diagonal and has $\mathcal{T}_{x x}=\mathcal{T}_{y y}=\left(n-\mathcal{T}_{z z}\right) / 2$. In contrast, the strong inhomogeneity of the 3D representation for a $1 \mathrm{D}$ system of $\mathrm{HR}, \rho(x, y, z)=\rho_{1 \mathrm{D}}(x) \delta(y) \delta(z)$, leaves a crucial role to the anisotropy of $\mathcal{T}(\boldsymbol{r})$ as the geometrical tool to obtain 
the complete cancellation of this third term for any 1D density distribution, so that the exact 1D result is recovered from the sum of the first two terms in $\Phi^{(3 \mathrm{D})}([\rho] ; \boldsymbol{r})$. Neglecting the anisotropy of $\mathcal{T}(\boldsymbol{r})$ in the oFMT produces the spurious divergence of $\phi_{3}^{(3 \mathrm{D})}(\eta, n, \boldsymbol{v})$ which invalidates the DF approximation for that dimensional reduction.

\subsubsection{Comparative Application of the Fundamental Measure Theory and the Weighted Density Approximation to a Spherical OD Cavity}

The application of different DF approximations to a simple 0D cavity offers an interesting perspective of their essential aspects. Let us consider a spherical density distribution, with total integral $\eta_{0}$, homogeneously distributed inside a small sphere of radius $\epsilon$. For any $\epsilon<R$ and $\eta_{0} \leq 1$, this density distribution represents a 0D cavity, with total excess free energy $\phi_{0}\left(\eta_{0}\right)=\left(1-\eta_{0}\right) \ln (1-$ $\left.\eta_{0}\right)+\eta_{0}$. This value should be obtained if the exact 3D excess free energy, $\beta \mathcal{F}_{\text {ex }}[\rho]$, were evaluated for the density distribution

$$
\rho(\boldsymbol{r})=\frac{3 \eta_{0}}{4 \pi \epsilon^{3}} \Theta(\epsilon-|\boldsymbol{r}|),
$$

with a radial step function of radius $\epsilon$. This is a very severe test for any DF approximation, particularly with respect to the singular behavior of $\phi_{0}\left(\eta_{0}\right)$, which has to reach the value $\phi_{0}(1)=1$ with infinite derivative, to represent the boundary between accessible and unaccessible density distributions of the HS within the cavity. The WDA represents the local density of excess free energy as $\Phi_{\mathrm{WDA}}=\rho(\boldsymbol{r}) \psi(\bar{\rho}(\boldsymbol{r}))$.

As presented in the left panel of Fig. 7.7 for a cavity of $\epsilon / \sigma=0.1$, the weighted density $\bar{\rho}(\boldsymbol{r})$ exceeds the range of $r=\sigma$, but only the values within $r \leq \epsilon$ are relevant for the evaluation of the free energy, through $\psi_{\mathrm{CS}}(\bar{\rho})$. In the left panel of Fig. 7.8, the free energy excess for low occupation is shown to be fairly independent of the cavity radius $\epsilon$ and recovers the exact quadratic dependence $\mathcal{F}_{\text {ex }}^{\mathrm{WDA}}[\rho]=\eta_{0}^{2} / 2+\mathcal{O}\left(\eta_{0}^{3}\right)$. For larger $\eta_{0}$, the CS-WDA results show some dependence on $\epsilon$, deviating from the exact $\phi_{0}\left(\eta_{0}\right)$. The representation of the boundary to unaccessible $\left(\eta_{0}>1\right)$ distributions in the WDA may only come from the lack of solutions for $\bar{\rho}(\boldsymbol{r})$, which in the CS-WDA occurs when the discriminant $\left(1-\bar{\rho}_{1}(\boldsymbol{r})\right)^{2}-4 \bar{\rho}_{0}(\boldsymbol{r}) \bar{\rho}_{2}(\boldsymbol{r})$ in $(7.40)$ vanishes. This is found to happen first at the center of the cavity, $r=0$, when the cavity occupation reaches a value $\eta_{0}=\eta_{\max }^{\mathrm{WDA}}$, which is marked by the full circles in Fig. 7.8. The WDA is not able to reproduce the exact result $\eta_{\max }=1$ for any $\epsilon<0.5 \sigma$, but taking into account that this DF approximation was designed using only information of the bulk liquid correlation structure, it is rewarding to observe that it gives a reasonable estimate for the accessible 0D states, ranging from $\eta_{\max }^{\mathrm{WDA}} \approx 0.8$ at $\epsilon=0$ to $\eta_{\max }^{\mathrm{WDA}} \approx 1.3$ at $\epsilon=R$. Moreover, the values of $\beta \mathcal{F}_{\mathrm{ex}}^{\mathrm{WDA}}[\rho]$ are reasonably close to the exact result, shown by the thick full lines in Fig. 7.8. The performance of the WDA for the description of the HS crystal is directly 

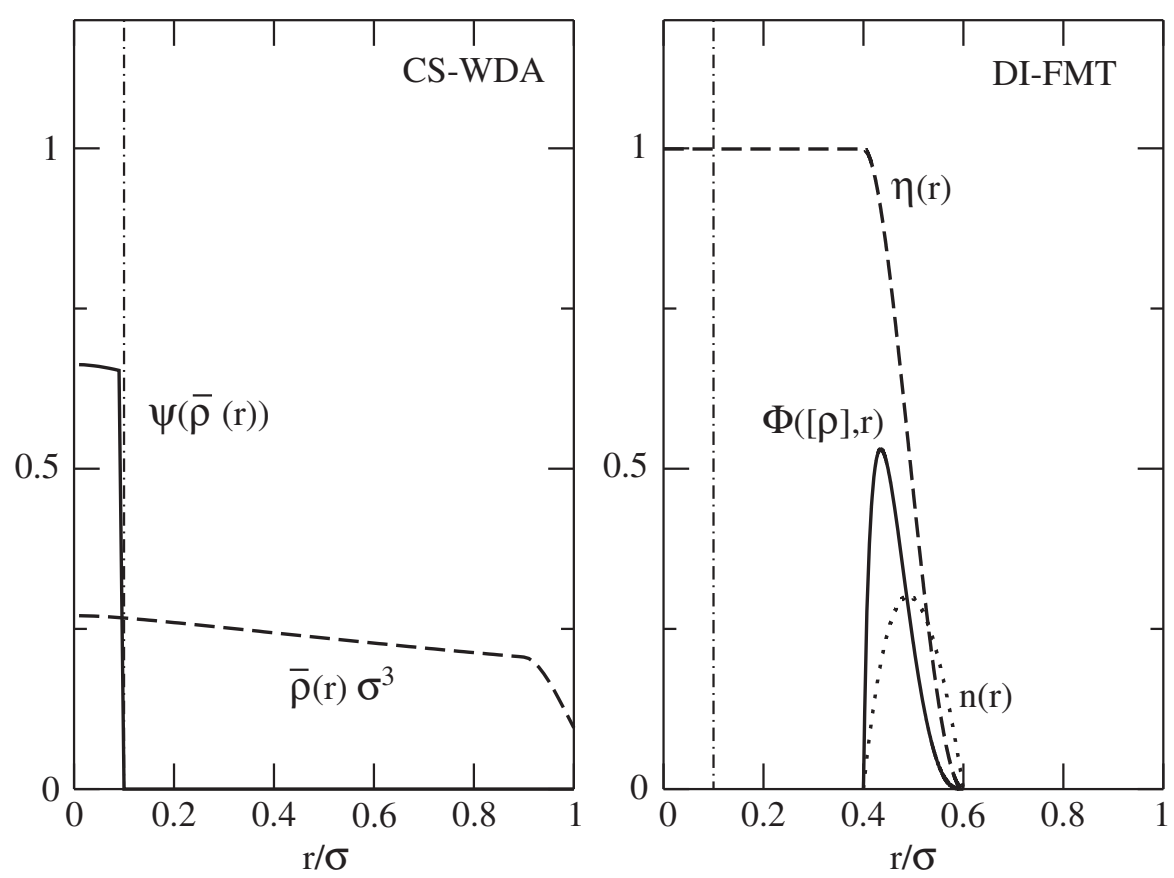

Fig. 7.7. Excess free energy density for a 0D cavity, with uniform density within a sphere of radius $\epsilon=0.1 \sigma$, marked by the vertical dash-dotted lines. The left panel shows the CS-WDA weighted density (dashed line) and the free energy excess per particle (full line) evaluated at $\bar{\rho}$, within the spherical density distribution, because that DF approximation assigns $\Phi([\rho], r)$ to the positions of the HS center; $\eta_{0}$ is taken close to its maximum allowed value. The right panel shows the main elements of the FMT for $\eta_{0}=1$, the local packing fraction (dashed line) is a smoothed radial step around $r=\sigma / 2$, with the inner value set by $\eta_{0}$. The scalar density $n(r)$ (dotted line) is restricted to the region $\sigma / 2-\epsilon<r<\sigma / 2+\epsilon$, and the same applies to the (not shown) vectorial and tensorial densities. Therefore, the excess free energy density (full line) is locally assigned to the positions of the HS surface

related to that for $0 \mathrm{D}$ cavities; when the unit cell occupancy is set to unity, the WDA estimation for the free energy is fairly good, because for $\eta_{0}=1$ the values of $\beta \mathcal{F}_{\mathrm{ex}}^{\mathrm{WDA}}[\rho]$ are not far from the exact $\phi_{0}(1)=1$. However, when the unit cell occupancy is used as a free variational parameter, the WDA result for the HS crystal is spoiled, because it is not able to locate the precise DF boundary at $\eta_{0}=1$.

In contrast, the FMT appears to have the precise non-local elements to extract the $0 \mathrm{D}$ limit, and it does it in a very different manner than the WDA, as shown in the right panel of Fig. 7.7. The local packing fraction $\eta(\boldsymbol{r})$ obtained from (7.73) reaches the value set by the total cavity occupancy $\eta_{0}$ for any $|\boldsymbol{r}| \leq R-\epsilon$, while the scalar, vector and tensor densities have non-zero values only over the range $R-\epsilon \leq|\boldsymbol{r}| \leq R+\epsilon$, so that the free energy excess is locally assigned to that region. The singularity of $\Phi^{\mathrm{FMT}}([\rho] ; \boldsymbol{r})$ at $\eta_{0}=1$ comes from the integration of the local free energy around $|\boldsymbol{r}|=R-\epsilon$, with vanishing combinations of $n(\boldsymbol{r}), \boldsymbol{v}(\boldsymbol{r})$ and $\mathcal{T}(\boldsymbol{r})$, divided by powers of $1-\eta(\boldsymbol{r})$. Both for the FMT based only on the OD limit and for the DI-FMT, the integration by parts of $\Phi^{\mathrm{FMT}}([\rho] ; \boldsymbol{r})$ recovers the singular behavior of the exact $\phi_{0}\left(\eta_{0}\right)$, 

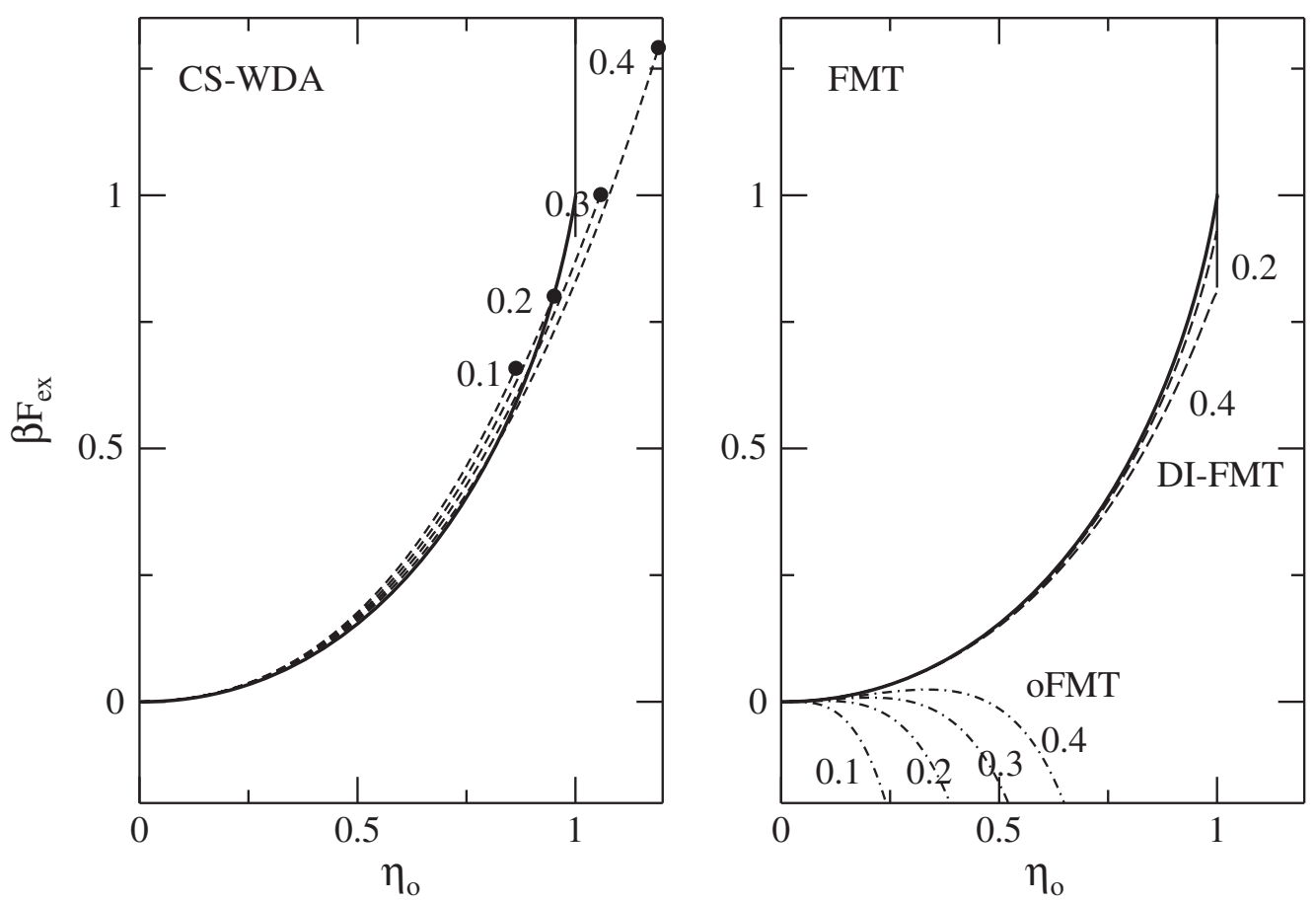

Fig. 7.8. Excess free energy, in $k T$ units, for $0 D$ spherical cavities with $\rho(r)=$ $3 \eta_{0} /\left(4 \pi \epsilon^{3}\right)$ for $r \leq \epsilon<\sigma / 2$ and $\rho(r)=0$ otherwise. The exact result (full line in both panels) is independent of $\epsilon$, and it is restricted to $\eta_{0} \leq 1$, as marked by the vertical lines. The dashed lines in the left panel show the CS-WDA results for $\epsilon / \sigma=0.1,0.2,0.3$ and 0.4 as labeled. The full circles mark the upper limit for $\eta_{0}$ set by the existence of real roots for equation (7.30). The right panel shows the results for the original FMT (dash-dotted lines) and the DI-FMT (dashed lines). The latter is very close to the exact results, so that only the results for $\epsilon / \sigma=0.2$ and 0.4 are presented. The oFMT presents qualitatively wrong negative values, diverging for $\eta_{0} \rightarrow 1$ at any $\epsilon$ and for $\epsilon \rightarrow 0$ at any $\eta_{0}$

with diverging slope at $\eta_{0}=1$, so that for any finite $\epsilon<R$, the boundary between accessible and unaccessible density distributions is exactly located. Nevertheless, there are small shifts, of order $\eta_{0}^{3} \epsilon^{2}$, which generate a weak dependence of the free energy excess with the cavity radius $\epsilon$. For the DIFMT results presented in the right panel of Fig. 7.8, that deviation is hardly visible at $\epsilon<0.2 \sigma$. The FMT version based only on the $0 \mathrm{D}$ limit would reduce this small discrepancy with the exact $\phi_{0}\left(\eta_{0}\right)$ but at the cost of a qualitative degradation of the results for the bulk HS fluid.

The oFTM proposed by Rosenfeld shows here a qualitative difference, because the contribution $\phi_{3}^{(3 \mathrm{D})}(\eta, n, \boldsymbol{v})$ in (7.68) has a negative divergence, proportional to $-\left[\epsilon\left(1-\eta_{0}\right)\right]^{-2}$, so that it ruins the good behavior of $\phi_{1}^{(3 \mathrm{D})}+$ $\phi_{2}^{(3 \mathrm{D})}$. As shown in Fig. 7.8, the results become even worse as $\epsilon$ decreases. From the results for the simple 0D cavity analyzed here, it becomes clear that the DF description of the HS crystal with the oFMT is bound to failure: the narrow Gaussian peaks describing the fluctuations of each particle around its lattice site would produce the same pathology in the evaluation of $\Phi^{\mathrm{FMT}}([\rho] ; \boldsymbol{r})$, and the minimization with respect to the Gaussian width would lead to a spurious negative divergence for delta-function peaks. 


\subsubsection{Application of the Fundamental Measure Theory to Planar Density Profiles: Practical Handling of Vector and Tensor Weights}

The application of the FMT to describe the density profiles of a HS fluid against a planar wall, or any other system with $\rho(\boldsymbol{r})=\rho(z)$, is technically similar in either the oFMT or the DI-FMT, and they also produce very similar results. The convolutions of the density profile adopt the simple forms

$$
\begin{aligned}
& \eta(z)=\pi \int_{-R}^{R} \mathrm{~d} z^{\prime} \rho\left(z+z^{\prime}\right)\left(R^{2}-z^{\prime 2}\right), \\
& n(z)=\int_{-R}^{R} \mathrm{~d} z^{\prime} \frac{\rho\left(z+z^{\prime}\right)}{2 R} .
\end{aligned}
$$

The only non-zero component of the vector density is

$$
v_{z}(z)=\int_{-R}^{R} \mathrm{~d} z^{\prime} \rho\left(z+z^{\prime}\right) \frac{z^{\prime}}{2 R^{2}},
$$

while the tensor weighted density becomes diagonal, with

$$
\mathcal{T}_{z z}(z)=\int_{-R}^{R} \mathrm{~d} z^{\prime} \rho\left(z+z^{\prime}\right) \frac{z^{\prime 2}}{2 R^{3}} \equiv n(z)-\frac{\eta(z)}{2 \pi R^{3}}
$$

and $\mathcal{T}_{x x}=\mathcal{T}_{y y}=\left(n-\mathcal{T}_{z z}\right) / 2 \equiv \eta(z) /\left(4 \pi R^{3}\right)$.

There are only three independent convolutions (7.74)-(7.76), carried over the restricted range $-R \leq z^{\prime} \leq R$, to get the components of the excess free energy density, $\phi_{1}^{(3 \mathrm{D})}=-n(z) \ln [1-\eta(z)], \phi_{2}^{(3 \mathrm{D})}=4 \pi R^{3}\left[n(z)^{2}-v_{z}(z)^{2}\right] /[1-$ $\eta(z)]$ and any of the two versions for the third term, either in the oFMT version (7.68)

$$
\phi_{3}^{(3 \mathrm{D})}=8 \pi^{2} R^{6} n(z) \frac{n(z)^{2} / 3-v_{z}(z)^{2}}{[1-\eta(z)]^{2}},
$$

or in the DI-FMT (7.72)

$$
\phi_{3}^{(3 \mathrm{D})}=3 \pi^{2} R^{6} \frac{\left[n(z)-\mathcal{T}_{z z}(z)\right]\left[n(z)^{2}-4 v_{z}(z)^{2}+3 \mathcal{T}_{z z}(z)^{2}\right]}{[1-\eta(z)]^{2}} .
$$

Therefore, the formal use of vector and tensor weighted densities for these planar profiles adds no extra practical difficulty to the FMT with respect to the WDA. As commented above, the original expression (7.78) is recovered from the DI-FMT (7.79) if we neglect the anisotropy of the tensor $\mathcal{T}$ while keeping its trace, $\mathcal{T}_{x x}=\mathcal{T}_{y y} \approx \mathcal{T}_{z z} \approx n / 3$. 
The integration of $\Phi([\rho] ; z)=\sum_{i=1}^{3} \phi_{i}^{(3 D)}$ is similar to that in (7.45), with the only difference that it has to be extended to the full region with $n(z) \neq 0$, which goes up to a distance $R=\sigma / 2$ beyond that with $\rho(z) \neq$ 0 . Thus, to get the excess free energy of a HS fluid against a hard wall, with $\rho(z)=0$ for $z<0$, the integral over the excess free energy has to be extended from $z=-R$ to cover all the region accessible to a HS surface, rather than accessible to its center. As in the WDA, we have to extend the integration beyond the maximum distance allowed for variable $\rho(z)$ in order to include the full range with $n(z) \neq \rho_{0}$. Altogether, the reduced range and the simpler analytical forms of the required density convolutions make the practical use of any of the FMT versions even easier than that of the WDA for planar density profiles. The subtleties of the delta-function convolutions disappear when applied to density distributions which only depend on $z$, and the two versions give similarly good results. In Fig. 7.9, we present a detailed view of the density profiles $\rho(z)$ for a HS fluid at bulk density $\rho_{B}=0.81 / \sigma^{3}$ against a hard wall. The computer simulation results by Snook and Henderson [21] (circles) are compared with the CS-WDA (dotted line) and several FMT versions. It is hard to distinguish the original formulation (oFMT, dashed line) from the dimensional interpolation version (DI-FMT, full line), and they both clearly improve the CS-WDA results in the interstitial region between the first two HS layers although the expanded view presented in this figure allows the observation of some deviations from the simulation data in the detailed shape the layer at $z \lesssim \sigma$, which would be hard to observe in the scale of Fig. 7.2. The DI-FMT results for the surface tension at a hard wall, as a function of the bulk HS density, are shown in Fig. 7.3. They are very similar to those of the

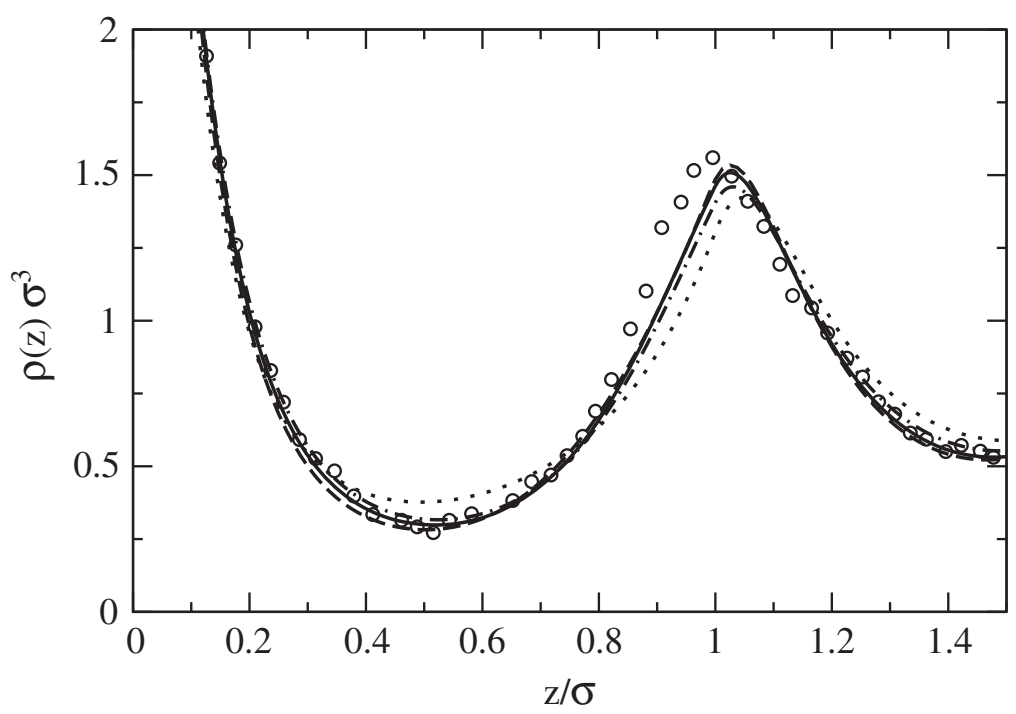

Fig. 7.9. Density profile for the HS fluid, with bulk density $\rho_{B}=0.81 / \sigma^{3}$ against a hard wall. The figure presents a detailed view of the central panel in Fig. 7.2. The computer simulations results [21] are compared with the CS-WDA (dotted line) and three FMT versions: oFMT (dashed line), DI-FMT (full line) and CS(WB)-FMT (dash-dotted line) 
oFMT, slightly above the SPT predictions, and within the error bars of the computer simulations.

\section{Dimensional Reduction to the 2D Hard Disks Fluid}

The most extreme inhomogeneity within the category of planar density profiles is the representation of the $2 \mathrm{D}$ homogeneous fluid of $\mathrm{HD}$ as $3 \mathrm{D}$ spheres with $\rho(z)=\rho_{2 \mathrm{D}} \delta(z)$. All the FMT weighted densities have the simple analytical forms derived from (7.74)-(7.77), and even the integrals of $\Phi_{i}^{(3 D)}([\rho] ; z)$ can be done analytically although their expressions are rather cumbersome [62]. All these DF approaches recover the first term in the generic DF virial expansion (7.16), and hence they give the exact value $\bar{B}_{2}=2$ in the virial expansion $\beta p / \rho_{2 \mathrm{D}}=1+\bar{B}_{2} \eta_{2 \mathrm{D}}+\bar{B}_{3} \eta_{2 \mathrm{D}}^{2}+\cdots$, with $\eta_{2 \mathrm{D}}=\pi \rho_{2 \mathrm{D}} \sigma^{2} / 4$. For the next term, the DI-FMT gives $\bar{B}_{3}=328 / 105=3.1238$, in very good agreement with the exact value $\bar{B}_{3}=3.128$, slightly above the SPT theory result $\bar{B}_{3}=3$. The CS-WDA $\left(\bar{B}_{3}=3.54\right)$ and Rosenfeld's oFMT $\left(\bar{B}_{3}=8 / 3=2.666\right)$ have larger deviations, with opposite signs.

However, the general aspect of $\beta p / \rho_{2 \mathrm{D}}$ at large $\rho_{2 \mathrm{D}}$, presented in Fig. 7.10, gives better overall results for the oFMT than for the DI-FMT. The quasiexact semi-empirical equation of state of Colot and Baus [63] appears between the SPT and the oFMT, while the DI-FMT deviates upward, but is still much better than the CS-WDA. It was shown in [62] that all the FMT versions produce a dependence $\beta p / \rho_{2 \mathrm{D}} \sim\left(1-\eta_{2 \mathrm{D}}\right)^{-5 / 2}$, which fits worse than the SPT form, $\beta p / \rho_{2 \mathrm{D}} \sim\left(1-\eta_{2 \mathrm{D}}\right)^{-2}$, to the density dependence of $\beta p / \rho_{2 \mathrm{D}}$ at large $2 \mathrm{D}$ fluid densities. Therefore, the excellent result of the oFMT comes from the fortunate cancellation of errors, between the underestimation of $\bar{B}_{3}$ for the

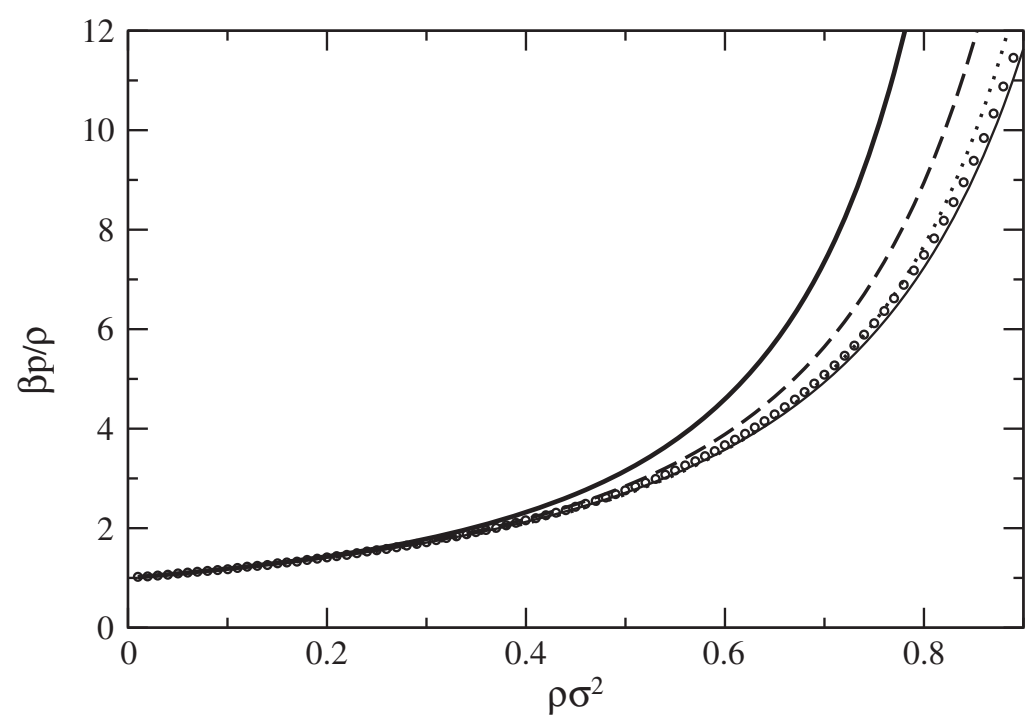

Fig. 7.10. The equations of state for the 2D hard disks fluid given by the SPT (thin full line) and the quasi-exact semi-empirical fit of Colot and Baus [63] (circles) are compared to those derived from the dimensional crossover of the free energy DF for 3D hard spheres: CS-WDA (thick full line), oFMT (dotted line) and DI-FMT (dashed line) 
low-density expansion and the overestimation of the ideal liquid divergence in the power of $1-\eta_{2 \mathrm{D}}$. The DI-FMT, built to interpolate between the 0D cavity and the 3D bulk fluid, goes exactly through the 1D free energy and gives an excellent result for $B_{3}$ in 2D, but it cannot keep the same quality at higher 2D densities. A different FMT version, with excellent interpolation between 0D, $1 \mathrm{D}$ and 2D, was also found [62], but it would give a rather poor estimation for the 3D equation of state. Altogether, we may say that the dimensional reduction from the DF for $3 \mathrm{D}$ HS to the equation of state of the $2 \mathrm{D}$ HD fluid is a good test for the DF dependence of $\Phi^{(3 \mathrm{D})}([\rho], r)$ and that the different FMT versions perform better than any WDA. Nevertheless, the best FMT versions are reaching the limits within that DF class, so that any consistent improvement would probably require the inclusion of a qualitatively different DF structure.

\subsubsection{Dimensional Interpolation Fundamental Measure Theory Applied to the Hard Sphere Crystal}

The application of the oFMT to describe the HS crystal was precluded by the spurious negative divergence of the $\Phi_{3}^{(3 \mathrm{D})}$ term. It was suggested [13] that this failure represented a fundamental flaw in the DF description of a crystal as a self-structured fluid, through the minimization of a density distribution like (7.50). The analysis of the $0 \mathrm{D}$ limit showed that the problem was directly associated to the negative divergence of $\Phi_{3}^{(3 \mathrm{D})}$, and an empirical modification of the oFMT was proposed $[64,65]$ to eliminate it and to recover a good description of the HS crystal within the FMT. However, it was only with the DI-FMT, and through the use of the tensor weight function, that the problem could be eliminated from its root, with the use of delta-function shell convolutions vanishing in the strict $0 \mathrm{D}$ limit. The practical application of the DI-FMT to density distributions made of Gaussian peaks is computationally more demanding than with the WDA, since the local distribution of excess free energy is assigned to the interstitial spaces, rather than being proportional to the narrow peaks in $\rho(\boldsymbol{r})$. Within the density parametrization (7.50), we may

obtain analytical expressions for the convolutions with a single, normalized, Gaussian peak centered at the origin. The scalar function is

$$
n^{\text {gauss }}(\boldsymbol{r})=\sqrt{\frac{\alpha}{\pi}} \frac{\mathrm{e}^{-\alpha(|\boldsymbol{r}|-R)^{2}}-\mathrm{e}^{-\alpha(|\boldsymbol{r}|+R)^{2}}}{4 \pi R|\boldsymbol{r}|} \approx \sqrt{\frac{\alpha}{\pi}} \frac{\mathrm{e}^{-\alpha(|\boldsymbol{r}|-R)^{2}}}{4 \pi R|\boldsymbol{r}|},
$$

where the second exponential term may be obviously neglected for any relevant value of $r \approx R$ and $\alpha \sigma^{2} \gg 1$. Within the same restrictions, the vector function is

$$
\boldsymbol{v}^{\text {gauss }}(\boldsymbol{r})=n^{\text {gauss }}(\boldsymbol{r})\left(1-\frac{1}{2 \alpha R|\boldsymbol{r}|}\right) \frac{\boldsymbol{r}}{|\boldsymbol{r}|},
$$

parallel to $\boldsymbol{r}$. The tensor components $\mathcal{T}_{\mu \nu}$, with $\mu, \nu=x, y, z$, take the form 


$$
\mathcal{T}_{\beta \gamma}^{\text {gauss }}(\boldsymbol{r})=n^{\text {gauss }}(\boldsymbol{r})\left[\frac{1-\xi(|\boldsymbol{r}|)}{2} \delta_{\beta \gamma}+\frac{3 \xi(|\boldsymbol{r}|)-1}{2} \frac{r_{\beta} r_{\gamma}}{|\boldsymbol{r}|^{2}}\right],
$$

with a diagonal term, and the direct product of the radial directions, given in terms of the function

$$
\xi(r)=1-\frac{1}{\alpha R r}+\frac{1}{2(\alpha R r)^{2}} .
$$

Notice that $\boldsymbol{v}^{\text {gauss }}(\boldsymbol{r})$ and $\mathcal{T}^{\text {gauss }}(\boldsymbol{r})$ have also exact (but more cumbersome) expressions to be used away from the practical restriction to $r \approx R$ and $\alpha \sigma^{2} \gg 1$. The local packing fraction takes the form

$$
\begin{aligned}
\eta^{\text {gauss }}(r, \alpha)= & \frac{3}{2}[\operatorname{erf}(\sqrt{\alpha}(R-|\boldsymbol{r}|))+\operatorname{erf}(\sqrt{\alpha}(R+|\boldsymbol{r}|)) \\
& \left.-\frac{\mathrm{e}^{-\alpha(R-|\boldsymbol{r}|)^{2}}-\mathrm{e}^{-\alpha(R+|\boldsymbol{r}|)^{2}}}{|\boldsymbol{r}| \sqrt{\pi \alpha}}\right],
\end{aligned}
$$

The contributions of all the Gaussian peaks on the crystal lattice have to be added, with fast convergence in terms of the distance to the point $\boldsymbol{r}$ to the lattice position. After adding all the relevant contributions to the vector and tensor cartesian components, the rotational invariant combinations in $(7.72)$ are evaluated. The final integral of $\Phi([\rho] ; \boldsymbol{r})$ has to be done much more carefully than for the WDA, since it extends to a narrow region on the lattice interstitial space and it contains inverse powers of $1-\eta(\boldsymbol{r})$ with (integrable) divergences in the strict limit of full unit cell occupancy. See [66] for a discussion of the present best numerical results.

The larger computational cost necessary to obtain results for the HS crystal with this DF approximation is clearly worthwhile because the equation of state of the HS crystal and its structure (represented by the optimal parameter $\alpha$ in (7.50)) are in excellent agreement with computer simulation results [61], as shown in Fig. 7.11, and certainly improve on the WDA results. Moreover, the extension of the DF minimization beyond the normalized Gaussian representation provides an even stronger support for the DI-FMT. The wider parametrization

$$
\rho(\boldsymbol{r})=\eta_{0}\left(\frac{\alpha}{\pi}\right)^{\frac{3}{2}} \sum_{\{\boldsymbol{R}\}} \mathrm{e}^{-\alpha|\boldsymbol{r}-\boldsymbol{R}|^{2}}[1+\tau \xi(\boldsymbol{r}-\boldsymbol{R})],
$$

where $\xi(\boldsymbol{r})=x^{4}+y^{4}+z^{4}-3|\boldsymbol{r}|^{4} / 5$ is the leading term in the anisotropy of the cubic lattices, was used to minimize $\Omega[\rho]$ with respect to $\eta_{0}, \alpha$ and $\tau$. The restriction to unit cell occupancy, $\eta_{0}=1$, which was essential for the accuracy of the WDA, is removed and the minimization of the DI-FMT free energy produces the self-adjusted normalization to $1-\eta_{0} \approx 10^{-8}$, i.e. a crystal with a negligibly small number of vacancies, well below the limit of observation in computer simulations (see the remarks on [67]). The anisotropy 

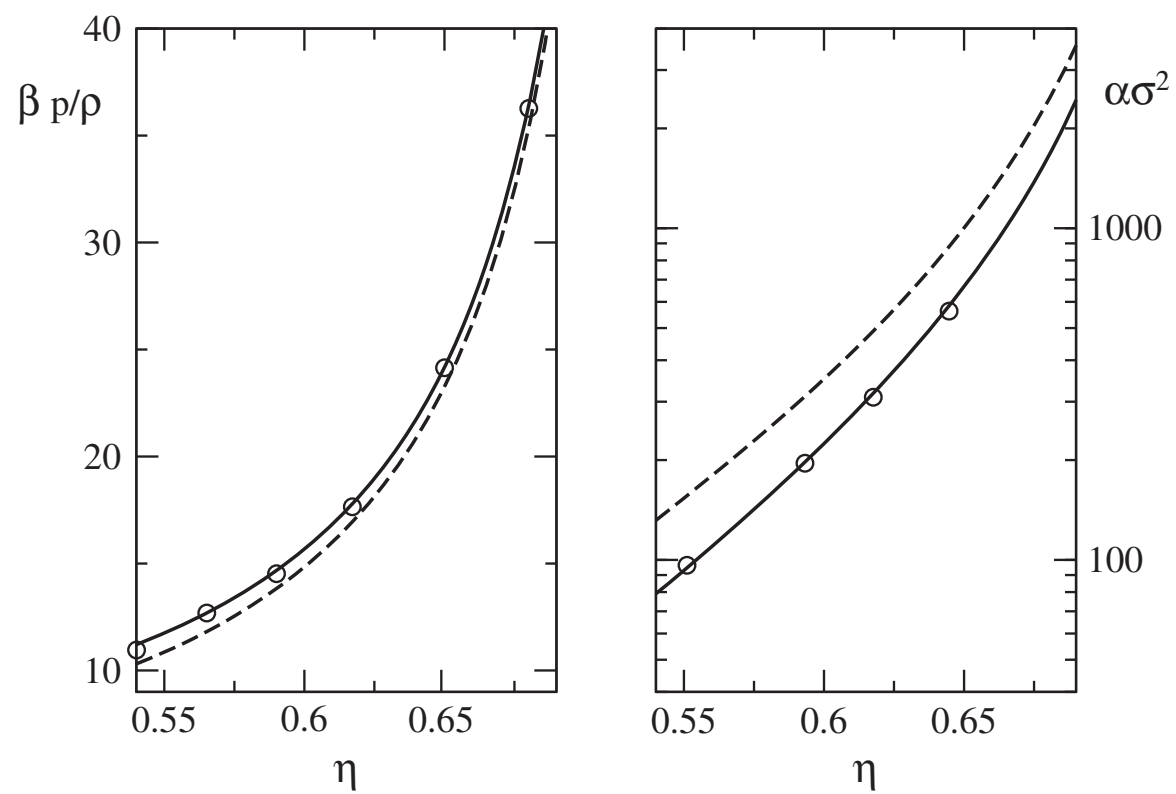

Fig. 7.11. The equation of state (left) and the square inverse Gaussian width parameter (right) for the HS FCC crystal, in terms of the mean packing fraction $\eta=\rho_{0} \pi \sigma^{3} / 6$. The full lines are the results of the DI-FMT, the dashed lines those of the CS-WDA and the circles present the computer simulation data from [32, 33].

of the density distribution was characterized with $\tau \approx 0.02$ for a crystal with mean density $\rho_{0}=1.03 / \sigma^{3}$, which is consistent with the computer simulation observation of a maximum spreading between 10 and $15 \%$ of the density along the interstitial $[1,1,1]$ direction, with respect to that of the nearest neighbor $[1,1,0]$ direction, of the FCC lattice. This contrasts with the opposite sign for the anisotropy $(\tau<0)$ predicted by the WDA, which would correspond to a small, but unphysical, accumulation of the density along the nearest neighbor directions [38].

As with the WDA, the DI-FMT does not discern between the free energy of the different closed packed structures. The FCC and HCP lattices have identical free energies, up to the present best numerical accuracy. However, the application to other (metastable) crystal structures marks a qualitative difference, again in favor of the DI-FMT. A HS crystal with BCC structure is unstable with respect to shears which would transform it into the FCC, but its DF characterization is still useful for the description of systems with soft repulsions from a HS reference system [68]. However, the WDA with normalized Gaussian peaks on a BCC lattice gives fully unphysical predictions, with the Gaussian peaks becoming wider as the density approaches its maximum compatible with the BCC structure. The use of the DI-FMT solves the problem, and it gives very reasonable predictions for the metastable BCC and even for the simple cubic, crystal structures [66].

Altogether, the representation of the HS crystal within the DI-FMT is excellent and qualitatively superior to the WDA or any other DF theory. In fact, the main practical problem of the DI-FMT to study the crystallization of HS comes from its unprecedented success in the representation of the HS crystal, 
since its equation of state is much more accurate than that of the compressibility PY approximation for the bulk HS fluid, which is taken as an input to design the DF approximation. The predicted densities of the coexisting fluid and crystal phases are rather poor, $\rho_{f}=0.892 / \sigma^{3}$ and $\rho_{c}=0.985 / \sigma^{3}$, far from the accuracy of the CS-WDA presented in Table 7.1, which makes more paired errors in the thermodynamics of the two phases. In the following subsection, we comment on an attempt to solve this puzzling situation, having a DF approximation which is too good in the description of the structured phase, compared with the (input) thermodynamics of the homogeneous liquid. However, we may anticipate that the question is still open, as a challenge for the future, in the amazing development of the FMT.

\subsubsection{Other Applications and Versions of the Fundamental Measure Theory}

Beside those which have already been commented, DF approximations within the FMT scheme have been applied to other interesting problems, e.g. the use of the test-particle route for the correlation structure [69] or fluids confined in narrow cylindrical or spherical cavities [70]. Also, we have to comment on several variants of the FMT which have been explored and used in different contexts, like that of Gonzalez et al. [71], who build a FMT version using only the two simplest elements: $\eta(\boldsymbol{r})$ and the scalar delta-function shell convolution $n(\boldsymbol{r})$. Their construction is based on the idea that $\phi_{0}\left(\eta_{0}\right)-1$ is the ideal free energy of a gas of holes (of density $1-\eta_{0}$ ) and it may be used as a generating functional. It is clear that such a DF approximation will have stronger unphysical divergences than the original FMT, since none of the delta-function shell overlaps are eliminated; however, all these divergences have positive sign, and the DF minimization of $\Omega[\rho]$ always avoids them. The quality of the results for systems with planar symmetry was clearly worse than with the oFMT, since avoiding the spurious divergences $\rho(\boldsymbol{r})$ develops unphysical kinks, but at least the results represent true minima of $\Omega[\rho]$, since $\mathcal{F}_{\text {ex }}[\rho]$ does not have the spurious bottomless pits for $0 \mathrm{D}$ cavities. An interesting feature of that simple FMT version was its flexibility to plug any bulk thermodynamics, like the CS equation of state which could improve on the PY approximation.

The idea of plugging the CS equation of state within the FMT is most appealing with the DI-FMT, since the excellent quality of the results for the crystal phase made (comparatively) bad the PY approximation for the bulk fluid. Along the lines of Gonzalez et al. [71], a CS version of the FMT was proposed and tested by Tarazona [62] maintaining the DI-FMT structure, but changing the $\eta$-dependence of $\phi_{3}^{(3 \mathrm{D})}$ in (7.72) to

$$
\begin{aligned}
\phi_{3}^{(3 \mathrm{D})}(\eta, n, \boldsymbol{v}, \mathcal{T})= & 12 \pi^{2} R^{6} \varphi_{\mathrm{CS}}(\eta(\boldsymbol{r})) \\
& \times\left(\boldsymbol{v} \cdot \mathcal{T} \cdot \boldsymbol{v}-n \boldsymbol{v} \cdot \boldsymbol{v}-\operatorname{Tr}\left[\mathcal{T}^{3}\right]+n \operatorname{Tr}\left[\mathcal{T}^{2}\right]\right),
\end{aligned}
$$


with

$$
\varphi_{\mathrm{CS}}(\eta)=\frac{2}{3 \eta^{2}}\left[\frac{\eta}{(1-\eta)^{2}}+\ln (1-\eta)\right]=1+\frac{16}{9} \eta+\frac{5}{2} \eta^{2}+\cdots
$$

instead of $\varphi_{\mathrm{PY}}(\eta)=(1-\eta)^{-2}=1+2 \eta+3 \eta^{2}+\cdots$ used in (7.72). This DI-FMT version, which we will refer to as the CS-FMT, was used to study the HS crystal and its dimensional reduction to the 2D fluid of HD [62]. The extension of this approximation to HS mixtures was later proposed by Roth et al. [72], under the name of the White Bear (WB) FMT. That name appears sometimes associated also to the mono-component case, which was used in the same paper as a check of the results in [62] for the HS crystal and to explore other problems of interest. The results of that CS(WB)-FMT are reasonably good, keeping all the qualitative advantages of the DI-FMT with respect to the WDA and improving the value of the coexisting fluid and crystal densities: $\rho_{f}=0.934 / \sigma^{3}$ and $\rho_{c}=1.023 / \sigma^{3}$. However, it is clear that the quality of the description for the HS crystal is degraded with respect to the (quasi-exact) results of the DI-FMT. Also in the application to planar density profiles, like the HS fluid against a hard wall shown in Fig. 7.9, the accuracy of the CS bulk equation of state is reflected in a better value for the contact density $\rho(0)=\beta p\left(\rho_{B}\right)$ given by the CS(WB)-FMT, but the effect is restricted to a very narrow range of distances to the wall, and for $z \approx \sigma$ the CS(WB)-FMT results are marginally worse than those of the DI-FMT or the oFTM.

Therefore, the adjustment of the free energy for the bulk fluid in (7.87) is done somehow against the natural structure of the FMT, changing the $1 /(1-\eta)^{2}$ dependence in $(7.72)$ which emanates from the third derivative of the exact $0 \mathrm{D}$ free energy $\phi_{0}(\eta)$. It was also noticed by Tarazona [62] that the low-density expansion of the PY approximation is exact, up to order $\rho_{0}^{2}$ (included), for any $|\boldsymbol{r}| \leq \sigma$. The first discrepancy between the PY and the exact $c\left(r, \rho_{0}\right)$ arises at order $\rho_{0}^{3}$ inside the core, but it already appears at order $\rho_{0}^{2}$ outside the core, where the tail for $\sigma<r \leq \sqrt{2} \sigma$ in the exact expansion is neglected by the PY assumption $c\left(r, \rho_{0}\right)=0$ for $r \geq \sigma$. The volume integral of this tail produces the difference between the fourth virial coefficients in the PY and the CS equations of state (bracketing the exact result), but within the $\mathrm{CS}(\mathrm{WB})-\mathrm{FMT}$ that difference is assigned to the core of $c\left(r, \rho_{0}\right)$ rather than to create a tail for $r>\sigma$.

Therefore, the empirical corrections leading to (7.86), or to the generic WB-FMT for HS mixtures, are giving the wrong non-local dependence to the thermodynamic improvement, beyond the PY approximation. The FMT appears to be the natural extension of the PY approximation for inhomogeneous HS fluids, with the assumption $\delta^{2} \mathcal{F}_{\text {ex }}[\rho] / \delta \rho(\boldsymbol{r}) \delta \rho\left(\boldsymbol{r}^{\prime}\right)=0$ for $\left|\boldsymbol{r}-\boldsymbol{r}^{\prime}\right| \geq \sigma$ emanating from the use of the $R$-ranged fundamental measures. Hence, it would be difficult to achieve a really consistent improvement of the FMT, with a better thermodynamics for the bulk fluid, without going beyond the use of one-center convolutions of the density. The analysis of HS mixtures presented in the next sections also supports the FMT as the most satisfactory framework 
to get very good DF approximations for hard core particles, but still with its intrinsic limitations, which seem difficult to overcome without a drastic transformation of the generic description of the non-local dependence in $\mathcal{F}_{\text {ex }}[\rho]$.

\subsection{Density Functionals for Hard Sphere Mixtures}

There are two obvious extensions of any DF approach to the HS model: including soft, and maybe attractive, potentials and studying mixtures of HS. For the former, it has already been commented that one way to deal with such models is to use a MFA for the attractive part of the interaction potential over a reference HS system (with possibly a temperature-dependent diameter) that accounts for the repulsive part. The latter, however, turns out to be more problematic, because we need special-purpose DFs to deal with mixtures of HS, and the DFs we have already studied for a mono-component fluid of HS are only half-way the final result. We need further insights to study mixtures.

When facing this problem, the situation is very different if one considers WDA-like or FMT-like DFs. While the WDA is the 'easiest' non-local DF theory for HS, extending it for mixtures becomes a headache. FMT, however, is a DF theory whose formulation for mixtures is rather straightforward, and (except for some subtleties that will be explained later) its construction adds no further complications to the theory. We will review both theories in what follows, but first let us discuss the modifications we have to make in the general scheme of DF theory in order to account for mixtures.

First of all, every component or species of an $m$-component inhomogeneous mixture will have its own density profile, $\rho_{\nu}(\boldsymbol{r}), \nu=1,2, \ldots, m$. To simplify notation, we introduce the vector

$$
\boldsymbol{\rho}(\boldsymbol{r})=\left(\rho_{1}(\boldsymbol{r}), \ldots, \rho_{m}(\boldsymbol{r})\right)
$$

to refer to all density profiles collectively. As for one-component systems, there exist a unique intrinsic free energy $\mathcal{F}[\boldsymbol{\rho}]$ which is a functional of the vector density $\boldsymbol{\rho}(\boldsymbol{r})$. If on particles of species $\nu$ acts an external potential $V_{\nu}(\boldsymbol{r})$ and there is a reservoir of particles with chemical potential $\mu_{\nu}$ for that species, the grand potential

$$
\Omega[\boldsymbol{\rho}]=\mathcal{F}[\boldsymbol{\rho}]+\sum_{\nu=1}^{m} \int \mathrm{d} \boldsymbol{r} \rho_{\nu}(\boldsymbol{r})\left[V_{\nu}(\boldsymbol{r})-\mu_{\nu}\right]
$$

reaches its absolute minimum for the equilibrium density profile vector $\boldsymbol{\rho}(\boldsymbol{r})$. This minimum principle leads to the set of Euler-Lagrange equations

$$
\frac{\delta \Omega[\boldsymbol{\rho}]}{\delta \rho_{\nu}(\boldsymbol{r})}=\frac{\delta \mathcal{F}[\boldsymbol{\rho}]}{\delta \rho_{\nu}(\boldsymbol{r})}+V_{\nu}(\boldsymbol{r})-\mu_{\nu}=0, \quad \nu=1, \ldots, m
$$


A similar reasoning to that of Sect. 7.2.1 leads to the DF for an ideal gas mixture

$$
\beta \mathcal{F}_{\text {id }}[\boldsymbol{\rho}]=\sum_{\nu=1}^{m} \int \mathrm{d} \boldsymbol{r} \rho_{\nu}(\boldsymbol{r})\left[\ln \rho_{\nu}(\boldsymbol{r})-1\right] .
$$

A remark is on purpose here. As in the one-component case, there is a thermal wavelength $\Lambda_{\nu}$ which is different for each component. When dealing with 'quenched' mixtures, the most common kind of mixture, for which the global composition of the system is fixed beforehand, we can ignore this wavelength, as we have done so far, and set it to 1 . This is what we will do henceforth. However, maintaining this factor is relevant when species are actually aggregates of particles whose composition is determined by chemical equilibrium. When describing this kind of systems, the thermal wavelength (or rather the thermal volume) is replaced by the internal partition function of an aggregate of species $\nu$, and its specific shape is most relevant for the equilibrium configuration of the system [73] (for an illustration of the dramatic effects this may have on the system, see $[74,75,76,77])$.

The ideal term contains the entropy of mixing, and the remaining contribution to a DF will be the excess free energy,

$$
\beta \mathcal{F}[\boldsymbol{\rho}]=\beta \mathcal{F}_{\mathrm{id}}[\boldsymbol{\rho}]+\beta \mathcal{F}_{\mathrm{ex}}[\boldsymbol{\rho}] \equiv \int \mathrm{d} \boldsymbol{r}\left\{\Phi_{\mathrm{id}}(\boldsymbol{\rho}(\boldsymbol{r}))+\Phi([\boldsymbol{\rho}] ; \boldsymbol{r})\right\}
$$

where $\Phi_{\mathrm{id}}(\boldsymbol{\rho})=\sum_{\nu} \rho_{\nu}\left(\ln \rho_{\nu}-1\right)$. The direct correlation function will now be a matrix, which can be obtained trough

$$
\left.\frac{\delta^{2} \beta \mathcal{F}_{\mathrm{ex}}[\boldsymbol{\rho}]}{\delta \rho_{\mu}\left(\boldsymbol{r}_{1}\right) \delta \rho_{\nu}\left(\boldsymbol{r}_{2}\right)}\right|_{\boldsymbol{\rho}(\boldsymbol{r})=\boldsymbol{\rho}_{0}}=-c_{\mu \nu}\left(r_{12}, \boldsymbol{\rho}_{0}\right), \quad \mu, \nu=1, \ldots, m,
$$

and which is related to the total correlation function $h_{\mu \nu}\left(r, \boldsymbol{\rho}_{0}\right)$ via the (matrix) Ornstein-Zernike equation

$$
h_{\mu \nu}\left(r, \boldsymbol{\rho}_{0}\right)=c_{\mu \nu}\left(r, \boldsymbol{\rho}_{0}\right)+\sum_{\lambda=1}^{m} \rho_{0, \lambda} \int \mathrm{d} \boldsymbol{r}^{\prime} c_{\mu \lambda}\left(r^{\prime}, \boldsymbol{\rho}_{0}\right) h_{\lambda \nu}\left(\left|\boldsymbol{r}-\boldsymbol{r}^{\prime}\right|, \boldsymbol{\rho}_{0}\right) .
$$

One final remark concerns polydisperse systems. In all expressions above, we have considered, for simplicity, multicomponent mixtures. Polydisperse mixtures are a special kind of mixtures in which there are virtually an infinity of species, labeled by a continuous parameter $s \in\left(s_{0}, s_{1}\right)$ ( $s$ may refer to size, shape, charge or whatever parameter or set of parameters that renders the mixture polydisperse). For these systems, $\rho_{\nu}(\boldsymbol{r})$ becomes a function $\rho(s ; \boldsymbol{r})$ and dependencies on $\boldsymbol{\rho}(\boldsymbol{r})$ become functional dependencies on that function. Hence, all expressions change accordingly and all sums on $\nu$ must be replaced by integrals on $s$. The resulting equations are formally the same, so we will only refer to this kind of mixtures when there are relevant issues to mention. 


\subsection{Weighted Density Approximation for Mixtures of Hard Spheres}

In 1990, Denton and Ashcroft proposed an extension of the WDA to binary mixtures [50] and applied it to determine freezing. The theory was one of the many conceivable generalization of the WDA for HS, and in its construction several simplifications were assumed. Although some of them may be questionable, the theory represents a reasonable trade off between flexibility and numerical tractability. The form we are presenting it here would be its formulation for an $m$-component mixture although, as it will be clear, applying the resulting DF to more than a few components (most of its applications are for just 2 components) is only for the braves.

Denton and Ashcroft's proposal for the excess free energy density is

$$
\Phi_{\mathrm{WDA}}([\boldsymbol{\rho}], \boldsymbol{r})=\sum_{\mu=1}^{m} \rho_{\mu}(\boldsymbol{r}) \psi\left(\bar{\rho}^{(\mu)}, \boldsymbol{x}\right),
$$

where $\boldsymbol{x}=\left(x_{1}, \ldots, x_{m}\right)$ is the composition vector whose components, $x_{\nu}=\rho_{\nu} / \rho, \rho=\sum_{\nu} \rho_{\nu}$, are the global values of the molar fractions of the species over the entire system of volume $V$, and $\psi(\rho, \boldsymbol{x})$ is the free energy per particle of a mixture of total density $\rho$ and composition vector $\boldsymbol{x}$. The effective total densities $\bar{\rho}^{(\mu)}, \mu=1, \ldots, m$, are defined through weighted convolutions of the density profiles

$$
\bar{\rho}^{(\mu)}(\boldsymbol{r})=\sum_{\nu=1}^{m} \int \mathrm{d} \boldsymbol{r}^{\prime} \rho_{\nu}\left(\boldsymbol{r}+\boldsymbol{r}^{\prime}\right) w_{\mu \nu}\left(r^{\prime} ; \bar{\rho}^{(\mu)}(\boldsymbol{r}), \boldsymbol{x}\right) .
$$

Weights are assumed to be normalized to 1 and symmetric $\left(w_{\mu \nu}=w_{\nu \mu}\right)$.

Now, as in the WDA for HS, determining the weights amounts to relate the second derivative of $\beta \mathcal{F}_{\text {ex }}[\boldsymbol{\rho}]$ of the uniform mixture with its direct correlation function, i.e.

$$
\left.\frac{\delta^{2} \beta \mathcal{F}_{\text {ex }}[\boldsymbol{\rho}]}{\delta \rho_{\mu}(\boldsymbol{r}) \delta \rho_{\nu}\left(\boldsymbol{r}^{\prime}\right)}\right|_{\boldsymbol{\rho}(\boldsymbol{r})=\boldsymbol{\rho}_{0}}=-c_{\mu \nu}\left(\left|\boldsymbol{r}-\boldsymbol{r}^{\prime}\right|, \boldsymbol{\rho}_{0}\right) .
$$

Before going any further, let us comment on the assumptions leading to this proposal. There are two main simplifications: one is the dependence on $\boldsymbol{x}$, rather than assuming a dependence on the whole set of effective densities $\bar{\rho}^{(\mu)}(\boldsymbol{r})$; the other one is to assume the same form $\psi(\rho, \boldsymbol{x})$ for all partial free energy per particle functions in the ansatz (7.95). The aim of both simplifications is to keep the complexity of the theory at a reasonable level. However, there is a rationale behind them: the approximation implies that each particle of the non-uniform mixture contributes to the excess free energy as if it interacted with an effective locally uniform mixture whose total density is different for each species. 
It turns out that, of the two assumptions made by Denton and Ashcroft, the most questionable is the constant composition hypothesis. A decade later, Davidchack and Laird made a version of this WDA without this assumption [78]. They replaced equation (7.95) by

$$
\Phi_{\mathrm{WDA}}([\boldsymbol{\rho}], \boldsymbol{r})=\sum_{\mu=1}^{m} \rho_{\mu}(\boldsymbol{r}) \psi\left(\overline{\boldsymbol{\rho}}^{(\mu)}(\boldsymbol{r})\right),
$$

where now $\psi(\boldsymbol{\rho})$ is the free energy per particle of a mixture with partial densities $\boldsymbol{\rho}=\left(\rho_{1}, \ldots, \rho_{m}\right)$, and the effective densities $\overline{\boldsymbol{\rho}}^{(\mu)}(\boldsymbol{r})=\left(\bar{\rho}_{1}^{(\mu)}, \ldots, \bar{\rho}_{m}^{(\mu)}\right)$, $\mu=1, \ldots, m$, are defined by the averages

$$
\bar{\rho}_{\nu}^{(\mu)}(\boldsymbol{r})=\int \mathrm{d} \boldsymbol{r}^{\prime} \rho_{\nu}\left(\boldsymbol{r}+\boldsymbol{r}^{\prime}\right) w_{\mu \nu}\left(r^{\prime} ; \overline{\boldsymbol{\rho}}^{(\mu)}(\boldsymbol{r})\right) .
$$

Not only is this version of the WDA for mixtures more symmetric, but it also leads to simpler equations for the weights. Because of the constraint on the composition, in the version of Denton and Ashcroft there appears a term proportional to the inverse volume of the system, $V^{-1}$, which is absent in the Davidchack and Laird formulation.

When equations (7.98) and (7.99) are used in (7.97), the resulting equations for the weights are (in Fourier space)

$$
\begin{aligned}
-\hat{c}_{\mu \nu}(k)= & \left(\frac{\partial \psi}{\partial \rho_{\mu}}+\frac{\partial \psi}{\partial \rho_{\nu}}\right) \hat{w}_{\mu \nu}(k)+\frac{\partial^{2} \psi}{\partial \rho_{\mu} \partial \rho_{\nu}} \sum_{\lambda=1}^{m} \rho_{\lambda} \hat{w}_{\mu \lambda}(k) w_{\lambda \nu}(k) \\
& +\frac{\partial \psi}{\partial \rho_{\mu}} \sum_{\lambda=1}^{m} \rho_{\lambda} \frac{\partial \hat{w}_{\mu \lambda}}{\partial \rho_{\nu}}(k) \hat{w}_{\lambda \nu}(k)+\frac{\partial \psi}{\partial \rho_{\nu}} \sum_{\lambda=1}^{m} \rho_{\lambda} \hat{w}_{\mu \lambda}(k) \frac{\partial \hat{w}_{\lambda \nu}}{\partial \rho_{\nu}}(k),
\end{aligned}
$$

where the dependence on $\boldsymbol{\rho}$ is implicitly assumed. Equation (7.100) represents a computational challenge: they form a system of partial differential equations very hard to solve even numerically. One could try the CS-WDA strategy, namely expanding $w_{\mu \nu}(r, \boldsymbol{\rho})$ in powers of the densities $\rho_{\lambda}$,

$$
w_{\mu \nu}(r, \boldsymbol{\rho})=w_{\mu \nu}^{(0)}(r)+\sum_{\lambda=1}^{m} \rho_{\lambda} w_{\mu \nu \lambda}^{(1)}(r)+\sum_{\lambda, \gamma=1}^{m} \rho_{\lambda} \rho_{\gamma} w_{\mu \nu \lambda \gamma}^{(2)}(r)+\cdots,
$$

and truncate at second order. The weights $w_{\mu \nu}^{(0)}(r), w_{\mu \nu \lambda}^{(1)}(r)$ and $w_{\mu \nu \lambda \gamma}^{(2)}(r)$ are determined by inserting the expansion (7.101) in equation (7.100) and replacing $\hat{c}_{\mu \nu}(k)$ by its density expansion to second order. This eliminates the problem of the partial derivatives with respect to the densities. If once the weights are determined, we evaluate the convolutions 


$$
\begin{aligned}
\bar{\rho}_{\mu \nu}^{(0)}(\boldsymbol{r}) & =\int \mathrm{d} \boldsymbol{r}^{\prime} \rho_{\nu}\left(\boldsymbol{r}+\boldsymbol{r}^{\prime}\right) w_{\mu \nu}^{(0)}\left(r^{\prime}\right), \\
\bar{\rho}_{\mu \nu \lambda}^{(1)}(\boldsymbol{r}) & =\int \mathrm{d} \boldsymbol{r}^{\prime} \rho_{\nu}\left(\boldsymbol{r}+\boldsymbol{r}^{\prime}\right) w_{\mu \nu \lambda}^{(1)}\left(r^{\prime}\right), \\
\bar{\rho}_{\mu \nu \lambda \gamma}^{(2)}(\boldsymbol{r}) & =\int \mathrm{d} \boldsymbol{r}^{\prime} \rho_{\nu}\left(\boldsymbol{r}+\boldsymbol{r}^{\prime}\right) w_{\mu \nu \lambda \gamma}^{(1)}\left(r^{\prime}\right),
\end{aligned}
$$

then the weighted densities are the solution to the (approximate) system of algebraic equations

$$
\bar{\rho}_{\nu}^{(\mu)}(\boldsymbol{r})=\bar{\rho}_{\mu \nu}^{(0)}(\boldsymbol{r})+\sum_{\lambda=1}^{m} \bar{\rho}_{\mu \nu \lambda}^{(1)}(\boldsymbol{r}) \bar{\rho}_{\lambda}^{(\mu)}(\boldsymbol{r})+\sum_{\lambda, \gamma=1}^{m} \bar{\rho}_{\mu \nu \lambda \gamma}^{(2)}(\boldsymbol{r}) \bar{\rho}_{\lambda}^{(\mu)}(\boldsymbol{r}) \bar{\rho}_{\gamma}^{(\mu)}(\boldsymbol{r}) .
$$

This problem is definitely simpler than the one posed by equation (7.100), but still not computationally trivial. We have a system of $m^{2}$ algebraic equations rather than a simple quadratic equation, and obtaining it requires the determination of $m(m+1) / 2$ weights $w_{\mu \nu}^{(0)}$ (they are symmetric), $m^{2}(m+1) / 2$ weights $w_{\mu \nu \lambda}^{(1)}$ and $m^{2}(m+1)^{2} / 2$ weights $w_{\mu \nu \lambda \gamma}^{(2)}$ (they are symmetric in $\mu, \nu$ and in $\lambda, \gamma)$, that makes a total of $m(m+1)^{2}(m+2) / 4$ weights. Just for a binary mixture, $m=2$, this amounts to computing 18 weights; for a ternary mixture this number rises to 60 weights! And then we need to compute convolutions of them all with the densities and solve the equations...

This is probably the reason why this theory has never been applied as such. Denton and Ashcroft explicitly mention the numerical difficulty of this extension of the WDA and switch to the so-called modified WDA (MWDA), an effective liquid theory, to study freezing in a binary mixture of HS [50]. Their results are quite good compared to simulations, specially if one takes into account that effective liquid theories entirely refuse to account for the fluid structure appropriately. A year later [79], they changed the theory and introduced the WDA at the level of the first-order direct correlation functions $c_{\mu}^{(1)}(\boldsymbol{r},[\boldsymbol{\rho}])=-\delta \beta \mathcal{F}_{\mathrm{ex}} / \delta \rho_{\mu}(\boldsymbol{r})$. This simplifies the equations for the weights to the extent of making them proportional to the direct correlation functions, so the resulting theory is much easier to apply. The results for the layering of a binary HS mixture near a hard wall that they obtained with this new version were reasonable although there were important discrepancies with respect to simulations. Davidchack and Laird did compute the weights of the WDA as presented here by numerically solving the system (7.100), but they did it just for illustration purposes because, in order to apply it to the freezing problem, they also resorted to the MWDA version of their theory. There are hybrid approaches [80] in which local effective densities are used in (7.98), but on the right-hand side of (7.99), these local densities are replaced by their averages. This leads to simpler equations for the weights and to slightly improved results for the density profiles of a binary HS mixture near a hard wall.

We will not pursue this approach any further. By now its computational complexity should be clear, even for few component mixtures; if the number 
of components is large it is hopeless and its applicability to study polydisperse mixtures is out of question. On the other hand, at present it is not worthwhile to invest more effort in this or similar approaches because FMT provides a much better alternative to study, not only mixtures with an arbitrary number of species, but even polydisperse mixtures. So let us see how FMT deals with mixtures.

\subsection{Fundamental Measure Theory for Mixtures of Hard Spheres}

Two key pieces in Rosenfeld's inspiration to create FMT were the 1D DF and SPT. Let us see what they tell us about mixtures. We shall begin with the 1D DF. The exact functional for an additive mixture of HR in a segment was obtained by Vanderlick et al. [81]. Appropriately written, its form is most revealing. Formally, it looks the same as the one derived by Percus for a one-component fluid,

$$
\Phi^{(1 \mathrm{D})}([\rho] ; x)=-n(x) \ln [1-\eta(x)] ;
$$

the difference lies in the definition of the weighted densities, which now is

$$
\begin{aligned}
& n(x)=\frac{1}{2} \sum_{\nu=1}^{m}\left[\rho_{\nu}\left(x+\sigma_{\nu} / 2\right)+\rho_{\nu}\left(x-\sigma_{\nu} / 2\right)\right], \\
& \eta(x)=\sum_{\nu=1}^{m} \int_{-\sigma_{\nu} / 2}^{\sigma_{\nu} / 2} \rho_{\nu}\left(x+x^{\prime}\right) \mathrm{d} x^{\prime} .
\end{aligned}
$$

In other words, the mixture is accounted for by introducing a weight function for each species and adding up all the resulting weighted densities.

Let us see now what we can learn from SPT. The excess free energy density (in $k_{\mathrm{B}} T$ units) provided by SPT can be written in the form [12, 23, 24, 55]

$$
\Phi=-\xi_{0} \ln \left(1-\xi_{3}\right)+\frac{\xi_{1} \xi_{2}}{1-\xi_{3}}+\frac{1}{24 \pi} \frac{\xi_{3}^{3}}{\left(1-\xi_{3}\right)^{2}},
$$

where the variables $\xi_{i}, i=0,1,2,3$ are geometrically weighted density averages,

$$
\xi_{i}=\sum_{\nu=1}^{m} \rho_{\nu} R_{\nu}^{(i)},
$$

with $R_{\nu}^{(0)}=1, R_{\nu}^{(1)}=R_{\nu}, R_{\nu}^{(2)}=4 \pi R_{\nu}^{2}$ and $R_{\nu}^{(3)}=4 \pi R_{\nu}^{3} / 3$ being 1 , the curvature radius, the surface and the volume of a sphere of diameter $R_{\nu}$, respectively. This suggests that a free energy functional should depend on weighted densities whose weights have locally the same geometrical meaning. 
Thus, Rosenfeld's proposal [13] for the weight functions was to define, for each species $\nu=1, \ldots, m$, the same normalized weighted densities as for HS, i.e.

$$
\begin{aligned}
& \eta_{\nu}(\boldsymbol{r})=\int \mathrm{d} \boldsymbol{r}^{\prime} \rho_{\nu}\left(\boldsymbol{r}+\boldsymbol{r}^{\prime}\right) \Theta\left(R_{\nu}-\left|\boldsymbol{r}^{\prime}\right|\right), \\
& \boldsymbol{v}_{\nu}(\boldsymbol{r})=\int \mathrm{d} \boldsymbol{r}^{\prime} \rho_{\nu}\left(\boldsymbol{r}+\boldsymbol{r}^{\prime}\right) \frac{\boldsymbol{r}^{\prime}}{R_{\nu}} \frac{\delta\left(R_{\nu}-\left|\boldsymbol{r}^{\prime}\right|\right)}{4 \pi R_{\nu}^{2}}, \\
& n_{\nu}(\boldsymbol{r})=\int \mathrm{d} \boldsymbol{r}^{\prime} \rho_{\nu}\left(\boldsymbol{r}+\boldsymbol{r}^{\prime}\right) \frac{\delta\left(R_{\nu}-\left|\boldsymbol{r}^{\prime}\right|\right)}{4 \pi R_{\nu}^{2}}
\end{aligned}
$$

With them, one can define six collective densities

$$
\begin{aligned}
\eta(\boldsymbol{r}) & =\sum_{\nu=1}^{m} \eta_{\nu}(\boldsymbol{r}), & n^{(2)}(\boldsymbol{r}) & =\sum_{\nu=1}^{m} 4 \pi R_{\nu}^{2} n_{\nu}(\boldsymbol{r}), \\
\boldsymbol{v}^{(2)}(\boldsymbol{r}) & =\sum_{\nu=1}^{m} 4 \pi R_{\nu}^{2} \boldsymbol{v}_{\nu}(\boldsymbol{r}), & n^{(1)}(\boldsymbol{r}) & =\sum_{\nu=1}^{m} R_{\nu} n_{\nu}(\boldsymbol{r}), \\
\boldsymbol{v}^{(1)}(\boldsymbol{r}) & =\sum_{\nu=1}^{m} R_{\nu} \boldsymbol{v}_{\nu}(\boldsymbol{r}), & n^{(0)}(\boldsymbol{r}) & =\sum_{\nu=1}^{m} n_{\nu}(\boldsymbol{r}),
\end{aligned}
$$

where the scaling factors are chosen such that in the limit of uniform densities we have $\eta(\boldsymbol{r}) \rightarrow \xi_{3}, n^{(2)}(\boldsymbol{r}) \rightarrow \xi_{2}, n^{(1)}(\boldsymbol{r}) \rightarrow \xi_{1}$ and $n^{(0)}(\boldsymbol{r}) \rightarrow \xi_{0}$. By repeating the same procedure that led to the functional (7.66)-(7.68), one can obtain Rosenfeld's original excess free DF as $\Phi_{\text {oFMT }}([\boldsymbol{\rho}], \boldsymbol{r})=\Phi_{1}([\boldsymbol{\rho}], \boldsymbol{r})+\Phi_{2}([\boldsymbol{\rho}], \boldsymbol{r})+$ $\Phi_{3}([\boldsymbol{\rho}], \boldsymbol{r})$, where $[13]$

$$
\begin{aligned}
& \Phi_{1}([\boldsymbol{\rho}], \boldsymbol{r})=-n^{(0)} \ln (1-\eta), \\
& \Phi_{2}([\boldsymbol{\rho}], \boldsymbol{r})=\frac{n^{(1)} n^{(2)}-\boldsymbol{v}^{(1)} \cdot \boldsymbol{v}^{(2)}}{1-\eta}, \\
& \Phi_{3}([\boldsymbol{\rho}], \boldsymbol{r})=\frac{1}{24 \pi} n^{(2)} \frac{n^{(2)} n^{(2)}-3 \boldsymbol{v}^{(2)} \cdot \boldsymbol{v}^{(2)}}{(1-\eta)^{2}} .
\end{aligned}
$$

Actually, Rosenfeld found his functional directly for mixtures in the form just presented. For later convenience and for computational simplicity, we will use a form involving only the normalized weighted densities (7.111),

$$
\begin{aligned}
& \Phi_{1}([\boldsymbol{\rho}], \boldsymbol{r})=-\sum_{\nu=1}^{m} n_{\nu} \ln (1-\eta), \\
& \Phi_{2}([\boldsymbol{\rho}], \boldsymbol{r})=2 \pi \sum_{\nu, \mu=1}^{m} R_{\nu} R_{\mu}\left(R_{\nu}+R_{\mu}\right) \frac{n_{\nu} n_{\mu}-\boldsymbol{v}_{\nu} \cdot \boldsymbol{v}_{\mu}}{1-\eta}, \\
& \Phi_{3}([\boldsymbol{\rho}], \boldsymbol{r})=8 \pi^{2} \sum_{\nu, \mu, \lambda=1}^{m} R_{\nu}^{2} R_{\mu}^{2} R_{\lambda}^{2} n_{\nu} \frac{\frac{1}{3} n_{\mu} n_{\lambda}-\boldsymbol{v}_{\mu} \cdot \boldsymbol{v}_{\lambda}}{(1-\eta)^{2}} .
\end{aligned}
$$


As its one-component counterpart, this DF can give very accurate density profiles of HS mixtures near a hard wall. As a matter of fact, because of its expressibility in terms of collective densities, it can be as easily applied to polydisperse mixtures. As such, it has been successfully used by Pagonabarraga et al. [82] to study size segregation at the adsorption profiles of a polydisperse mixture of HS near a hard wall, something inconceivable with a WDA. But as it comes to study freezing, this functional has the same divergences that cause the breakdown of the solid minimization for the one-component HS fluid.

With an exception that we will comment later on, fixing these divergences requires the same strategy as the one followed for the one-component FMT. In fact, when expressed in terms of the collective densities, the resulting functionals are formally the same. Thus, apart from the empirical corrections introduced to avoid these singularities [64, 65] (and which have been applied with success to study entropic selectivity in microporous materials [83]), we can formulate an analog of the DI-FMT by introducing the new tensor weighted density

$$
\left(\mathcal{T}_{\nu}\right)_{\alpha \beta}(\boldsymbol{r})=\int \mathrm{d} \boldsymbol{r}^{\prime} \rho_{\nu}\left(\boldsymbol{r}+\boldsymbol{r}^{\prime}\right) \frac{r_{\alpha}^{\prime} r_{\beta}^{\prime}}{R_{\nu}^{2}} \frac{\delta\left(R-\left|\boldsymbol{r}^{\prime}\right|\right)}{4 \pi R_{\nu}^{2}}
$$

and replacing $\Phi_{3}$ of the oFMT by

$$
\begin{aligned}
\Phi_{3}([\boldsymbol{\rho}], \boldsymbol{r}) & =12 \pi^{2} \sum_{\nu, \mu, \lambda=1}^{m} R_{\nu}^{2} R_{\mu}^{2} R_{\lambda}^{2} \frac{\varphi_{\nu \mu \lambda}(\boldsymbol{r})}{[1-\eta(\boldsymbol{r})]^{2}} \\
\varphi_{\nu \mu \lambda}(\boldsymbol{r}) & =\boldsymbol{v}_{\nu} \cdot \mathcal{T}_{\mu} \cdot \boldsymbol{v}_{\lambda}-n_{\mu} \boldsymbol{v}_{\nu} \cdot \boldsymbol{v}_{\lambda}-\operatorname{Tr}\left(\mathcal{T}_{\nu} \mathcal{T}_{\mu} \mathcal{T}_{\lambda}\right)+n_{\mu} \operatorname{Tr}\left(\mathcal{T}_{\nu} \mathcal{T}_{\lambda}\right)
\end{aligned}
$$

The fact that the coefficient in (7.120) is a polynomial in the radii allows for an alternative form in terms of collective densities. Thus, if

$$
\mathcal{T}(\boldsymbol{r})=\sum_{\nu=1}^{m} 4 \pi R_{\nu}^{2} \mathcal{T}_{\nu}(\boldsymbol{r}),
$$

then (7.120) and (7.121) can be rewritten as

$$
\Phi_{3}([\boldsymbol{\rho}], \boldsymbol{r})=\frac{3}{16 \pi} \frac{\boldsymbol{v}^{(2)} \cdot \mathcal{T} \cdot \boldsymbol{v}^{(2)}-n^{(2)} \boldsymbol{v}^{(2)} \cdot \boldsymbol{v}^{(2)}-\operatorname{Tr}\left(\mathcal{T}^{3}\right)+n^{(2)} \operatorname{Tr}\left(\mathcal{T}^{2}\right)}{(1-\eta)^{2}}
$$

But if one checks dimensional crossover with this new functional, one encounters the surprise that it does not even recover the exact 1D limit! It is surprising because its one-component counterpart does. The reason is that the third term is not zero in this dimensional reduction. In order to understand what is going on, we should imagine situations in which the centers of three spheres are aligned and their three surfaces intersect at a common circle. For spheres of the same size, this can only occur if at least two spheres are at the same position. This means that their surfaces fully overlap, and the correction 
introduced by the DI-FMT DF is such that the third term vanishes when this happens. However, if the spheres are different, such a triple intersection can occur and the three spheres can sit at different positions. The DI-FMT DF does not account for these situations and so they do contribute to the third term. In [84], we considered this problem and showed that it can be fixed by adding a rank-3 tensor weighted density

$$
\left(\mathcal{M}_{\nu}\right)_{\alpha, \beta, \gamma}(\boldsymbol{r})=\int \mathrm{d} \boldsymbol{r}^{\prime} \rho_{\nu}\left(\boldsymbol{r}+\boldsymbol{r}^{\prime}\right) \frac{r_{\alpha}^{\prime} r_{\beta}^{\prime} r_{\gamma}^{\prime}}{R_{\nu}^{3}} \frac{\delta\left(R_{\nu}-\left|\boldsymbol{r}^{\prime}\right|\right)}{4 \pi R_{\nu}^{2}}
$$

and correcting $\varphi_{\nu \mu \lambda}(\boldsymbol{r})$ in (7.121) with the addition of

$$
\Delta \varphi_{\nu \mu \lambda}(\boldsymbol{r})=\frac{2 R_{\mu}^{2}\left(R_{\nu}-R_{\lambda}\right)}{R_{\nu}\left(R_{\nu}+R_{\mu}\right)\left(R_{\mu}+R_{\lambda}\right)}\left(\boldsymbol{v}_{\nu} \cdot \mathcal{M}_{\mu}: \mathcal{T}_{\lambda}-\boldsymbol{v}_{\nu} \cdot \mathcal{T}_{\lambda} \cdot \boldsymbol{v}_{\mu}\right)
$$

(the symbol ':' denotes the contraction of two indices). The vanishing of this term for a one-component fluid is patent from the factor $R_{\nu}-R_{\lambda}$. Also, it gives no contribution for uniform fluids because then $v_{\nu}(\boldsymbol{r})=0$ and $\mathcal{M}_{\nu \mu \lambda}(\boldsymbol{r})=0$. One striking feature is that the coefficient dependent on the radii is no longer a polynomial, but a rational function. The dramatic consequence of this is that the new functional cannot be written in terms of a finite number of collective densities, as the previous ones, thus spoiling one very nice feature of FMT, specially when applied to polydisperse mixtures. The difficulty in applying this functional with respect to the DI-FMT one is considerable, so it can only be justified if the gain in accuracy is enough.

In [84], density profiles of a binary HS mixture near a hard wall and within a narrow slit were obtained with both the corrected functional and the DI-FMT one. The profiles are shown in Figs. 7.12 and 7.13. As it can be seen, the differences between the two DFs are negligible. Also the comparison between the dimensional reduction to 1D of the DI-FMT and the corrected one (which is exact) shows, again for a binary mixture, that the differences are smaller than $2 \%$ for all packing fractions up to $\eta=0.8$ and is not larger than $10 \%$ for such a high packing fraction as $\eta=0.95$. The conclusion so far is that the higher complexity of the corrected FMT does not justify using it instead of the DI-FMT one, which is not only much simpler in that it contains no rank-3 tensor, but also in that it can be written in terms of collective variables, which gives a considerable advantage when studying polydisperse mixtures. It remains to study what we could call the worst case scenario: namely, inhomogeneous 1D-like situations like cylindrical pores. As far as we know, nobody has tested these two functionals in this situation yet.

Irrespective of this analysis, no matter whether we consider the DI-FMT or the corrected one, the problem of the lost cases commented on in Sect. 7.5.2 is even worse for mixtures than it is for a one-component fluid [84]. The reason is the inability of these FMT functionals to recover the lowest order in a density expansion of the third-order direct correlation function. As already mentioned, circumventing this problem would amount to modifying the structure of FMT 


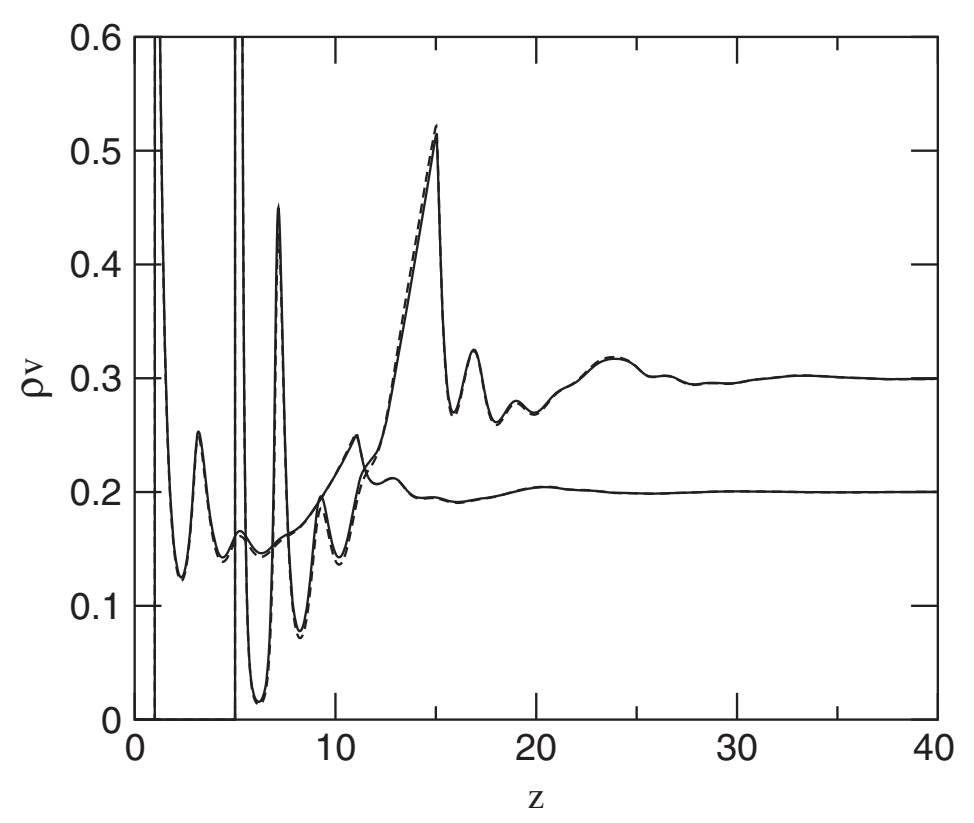

Fig. 7.12. Density profiles of the two components of a binary mixture of radii $R_{1}=1, R_{2}=5$ near a hard wall (the density is scaled with the volume of each sphere, denoted $v$ in the axis label). With a dashed line we plot the results of the DI-FMT, with a full line we plot the results of the corrected FMT functional

functionals dramatically, and it is not clear at all whether such modifications, even if they corrected the problem, would be computationally amenable. So this seems to be as far as FMT can go.

Nevertheless, empirical modifications of FMT have proposed that, even though they necessarily spoil some of their nice features, they can still produce very good results when applied to specific problems. As already mentioned in Sect. 7.4.3, one of the obvious modifications is to replace the SPT excess free

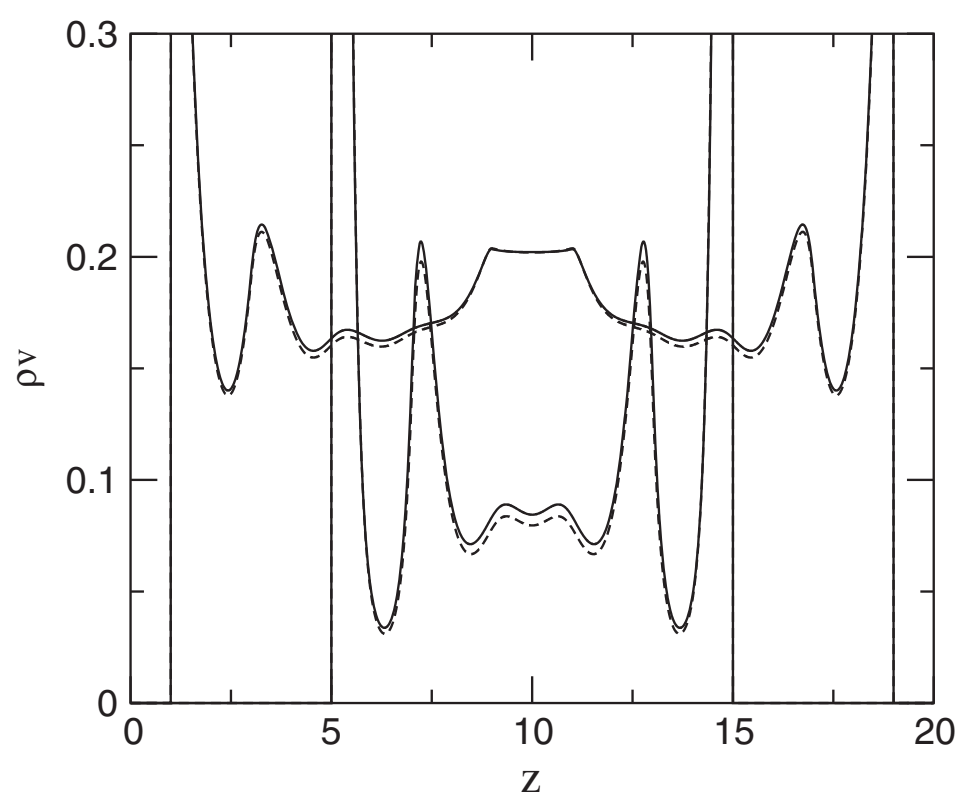

Fig. 7.13. Same as Fig. 7.12 for a slit of width $20 R_{1}$ 
energy density by the empirical BMCSL (from Boublík-Mansoori-CarnahanStarling-Leland). This has been done for the oFMT version $[72,85]$ as well as for the DI-FMT version [72]. The results for the density profiles of binary mixtures near a hard wall are in excellent agreement with simulations. When applied to polydisperse mixtures [86] they even improve those obtained with the original Rosenfeld's functional [82]. Finally, another empirical modification based on the CS free energy density [87] has been applied with excellent results to the calculation of the surface tension and bending rigidities of $\mathrm{HS}$ near a spherical surface (although the fluid is mono-disperse in this case, the analytical expressions derived for these magnitudes require the DF for a binary mixture, where one of the components becomes the spherical surface). Thus we see that, as it comes to concrete applications, FMT can still tolerate enough flexibility to gain in accuracy.

\subsection{Non-Additive Mixtures of Hard Spheres}

So far we have only considered ordinary, or additive, HS mixtures, but this is not the only interesting model of HS mixtures, there is a more general class of them which are non-additive. This means that, although the interaction between particles of either the same or different species is hard core, the effective diameters are related through the relationship

$$
\sigma_{\nu \mu}=\frac{1}{2}\left(\sigma_{\nu \nu}+\sigma_{\mu \mu}\right)\left(1+\Delta_{\nu \mu}\right) .
$$

The case $\Delta_{\nu \mu}=0$ for $\nu \neq \mu$ describes the situation for additive HS and the cases $\Delta_{\nu \mu}>0$ or $\Delta_{\nu \mu}<0$ describe non-additive HS. Such a model describes well certain mixtures of chemical compounds or mixtures of colloidal particles and polymers.

In spite of the apparent minor modification we have made, the resulting model is extraordinarily more difficult than the additive one, so much that there is no known functional for a single non-additive HS mixture even in 1D. Just to illustrate the problem, one must bear in mind that, while additive HR in $1 \mathrm{D}$ remain mixed for all densities up to close packing, if the mixture is nonadditive with $\Delta_{\nu \mu}>0$, the mixed state is entropically disfavored with respect to the mixed one, so that close packing is only attainable for a fully demixed state. This fact forces the system to undergo a glass transition because, upon increasing density, the system gets trapped in smaller regions of the phase space as soon as some mixed configuration stops being reachable for that given density [88]. Thus, non-additive HS mixtures bring about a higher complexity than additive ones.

Because of this, there are fewer DFs for non-additive HS mixtures than there are for additive ones. Nevertheless we should mention a few exceptions in which functionals in the FMT spirit have been proposed, although to obtain them some of the nice features of this theory have had to be sacrificed. We 
will mostly describe two of them, plus an attempt at generalizing FMT to non-additive HS mixtures with arbitrary $\Delta_{\nu \mu}$.

\subsubsection{The Asakura-Oosawa-Vrij Model}

Let us consider the following simplified model of a colloid-polymer mixture introduced first by Asakura and Oosawa [89] and later by Vrij [90]. Colloidal particles have a typical radius $R_{c}$ and polymers $R_{p}$. Interaction between colloidal particles is HS-like with diameter $\sigma_{c c}=2 R_{c}$, between colloidal particles and polymers is also HS-like with diameter $\sigma_{c p}=R_{c}+R_{p}$, but polymers are ideal particles to themselves (i.e. $\sigma_{p p}=0$ ). This is a non-additive HS mixture with $\Delta_{c p}=R_{p} / R_{c}>0$. For this model, Schmidt et al. [91] proposed the following functional. They kept the general FMT scheme, so their functional is formally the same as (7.116), (7.117), (7.120), (7.121),

$$
\begin{aligned}
\Phi_{1}([\boldsymbol{\rho}], \boldsymbol{r})= & \sum_{\nu=c, p} n_{\nu} \phi_{\nu}\left(\eta_{c}, \eta_{p}\right) \\
\Phi_{2}([\boldsymbol{\rho}], \boldsymbol{r})= & 2 \pi \sum_{\nu, \mu=c, p} R_{\nu} R_{\mu}\left(R_{\nu}+R_{\mu}\right)\left[n_{\nu} n_{\mu}-\boldsymbol{v}_{\nu} \cdot \boldsymbol{v}_{\mu}\right] \phi_{\nu \mu}\left(\eta_{c}, \eta_{p}\right) \\
\Phi_{3}([\boldsymbol{\rho}], \boldsymbol{r})= & 8 \pi^{2} \sum_{\nu, \mu, \lambda=c, p} R_{\nu}^{2} R_{\mu}^{2} R_{\lambda}^{2}\left[\boldsymbol{v}_{\nu} \cdot \mathcal{T}_{\mu} \cdot \boldsymbol{v}_{\lambda}-n_{\mu} \boldsymbol{v}_{\nu} \cdot \boldsymbol{v}_{\lambda}\right. \\
& \left.-\operatorname{Tr}\left(\mathcal{T}_{\nu} \mathcal{T}_{\mu} \mathcal{T}_{\lambda}\right)+n_{\mu} \operatorname{Tr}\left(\mathcal{T}_{\nu} \mathcal{T}_{\lambda}\right)\right] \phi_{\nu \mu \lambda}\left(\eta_{c}, \eta_{p}\right)
\end{aligned}
$$

where the weighted densities $\eta_{\nu}(\boldsymbol{r}), n_{\nu}(\boldsymbol{r}), \boldsymbol{v}_{\nu}(\boldsymbol{r})$ and $\mathcal{T}_{\nu}(\boldsymbol{r}), \nu=c, p$, are defined as in (7.111), (7.119) and

$$
\phi_{\nu_{1} \cdots \nu_{l}}\left(\eta_{c}, \eta_{p}\right)=\frac{\partial^{l} \phi_{0}\left(\eta_{c}, \eta_{p}\right)}{\partial \eta_{\nu_{1}} \cdots \partial \eta_{\nu_{l}}},
$$

$\phi_{0}\left(\eta_{c}, \eta_{p}\right)$ being the excess free energy of a $0 \mathrm{D}$ cavity.

If the colloidal particles and polymers were just additive HS, then the grand partition function of a $0 \mathrm{D}$ cavity would be

$$
\Xi_{0}=1+z_{c}+z_{p}
$$

with $z_{c}, z_{p}$ their respective fugacities. Then $\eta_{\nu}=z_{\nu} / \Xi_{0}$, from which $\Xi_{0}^{-1}=$ $1-\eta_{c}-\eta_{p}$ and therefore

$$
\begin{aligned}
\phi_{0}\left(\eta_{c}, \eta_{p}\right) & \equiv \eta_{c}+\eta_{p}-\ln \Xi_{0}+\eta_{c} \ln \left(z_{c} / \eta_{c}\right)+\eta_{p} \ln \left(z_{p} / \eta_{p}\right) \\
& =\eta_{c}+\eta_{p}+\left(1-\eta_{c}-\eta_{p}\right) \ln \left(1-\eta_{c}-\eta_{p}\right) .
\end{aligned}
$$

Substituting this function back into (7.127)-(7.129), one recovers the DI-FMT for an additive HS mixture (7.116), (7.117), (7.120), (7.121).

But if particles are like in the Asakura-Oosawa-Vrij (AOV) model, then

$$
\Xi_{0}=z_{c}+e^{z_{p}}
$$


because in a $0 \mathrm{D}$ cavity there may be one colloidal particle or an arbitrary number of polymers. Therefore,

$$
\eta_{c}=\frac{z_{c}}{\Xi_{0}}, \quad \eta_{p}=\frac{z_{p} e^{z_{p}}}{\Xi_{0}},
$$

so $z_{c}=\eta_{c} \Xi_{0}$, from $(7.133) e^{z_{p}}=\Xi_{0}\left(1-\eta_{c}\right)$ and thus

$$
z_{p}=\frac{\eta_{p}}{1-\eta_{c}}, \quad \Xi_{0}=\frac{\exp \left(\frac{\eta_{p}}{1-\eta_{c}}\right)}{1-\eta_{c}}, \quad z_{c}=\frac{\eta_{c} \exp \left(\frac{\eta_{p}}{1-\eta_{c}}\right)}{1-\eta_{c}} .
$$

The excess free energy that follows from these expressions is

$$
\phi_{0}\left(\eta_{c}, \eta_{p}\right)=\eta_{c}+\left(1-\eta_{c}-\eta_{p}\right) \ln \left(1-\eta_{c}\right) .
$$

The functional (7.127)-(7.129) possesses a set of good properties: (i) by construction (it has the same structure as the DI-FMT), it recovers the correct $0 \mathrm{D}$ limit for $0 \mathrm{D}$ cavities with no lost cases; (ii) the first two terms in the low-density limit of the direct correlation function are exact; (iii) it generates the correct depletion potential between two colloidal particles in a sea of ideal polymers at any density [92], and (iv) it is linear in $\eta_{p}(\boldsymbol{r})$. The latter has some important implications: on the one hand, the direct correlation function $c_{p p}\left(r, \rho_{c}, \rho_{p}\right)=0$ as in the PY approximation; on the other hand, the Euler-Lagrange equation for $\rho_{p}(\boldsymbol{r})$ permits to obtain explicitly this density as a functional of only $\rho_{c}(\boldsymbol{r})$, something that greatly simplifies the minimization process. As a matter of fact, this nice feature can be used to obtain explicitly a DF for the effective fluid of colloidal particles interacting with the depletion potential induced by the polymers. This can be achieved through the procedure described in [93].

As for the results one can get from this functional, for uniform densities $[91,94]$ the resulting free energy coincides with that of the free-volume approximation of the AOV model [95], which predicts a stable gas-liquid coexistence for $\Delta_{c p} \gtrsim 0.32$. For $\Delta_{c p}<0.1547$, the model can be exactly mapped onto a one-component colloidal fluid with pairwise interaction [96]. This allows to perform simulations for such an asymmetric mixture that would otherwise be hindered by severe equilibration problems. The comparison of the structure factors is quite good [91].

A variant of this model in which a third component, point-like HS, mimics a poor solvent can also be dealt with in the same manner [97]. The model exhibits a richer phenomenology, with a reentrant demixing transition for a sufficiently high solvent concentration.

The procedure sketched here is a very ingenious twist of the standard FMT for additive HS. Its results are very good, considering the simplicity of the proposal and the difficulty of the model. This notwithstanding, there is an important and unavoidable flaw in this extension of FMT to non-additive models: the 1D limit cannot be correctly recovered. Having in mind the dimensional crossover behavior of the DI-FMT, it is obvious that, for $\Delta_{\nu \mu}=1$, the 
1D limit of this AOV DF will be just $\Phi_{1}$ in (7.127), a result that is only a crude approximation to the exact excess free energy of the 1D AOV model [88]. Even worse, if $\Delta_{\nu \mu} \neq 1$, then the term $\Phi_{3}$ in (7.129) also makes a contribution for the same reasons that it does for an additive HS mixture (see Sect. 7.8) [94]. Actually, this problem can be fixed by patching out the DF in a way similar to that employed in Sect. 7.8, i.e. adding a contribution like (7.125) to the term (7.129). Notice that this will change neither the excess free energy nor the direct correlation function of the uniform fluid, so all features of the AOV FMT described above remain intact; only the 1D limit will change and will then be given by (7.127) for any particle radii.

\subsubsection{The Widom-Rowlinson Model}

Another model whose DF can be obtained with the same procedure is the Widom-Rowlinson model [98]. This is an $m$-component non-additive HS mixture of radii $R_{\nu}, \nu=1, \ldots, m$ such that

$$
\sigma_{\nu \mu}=R_{\nu}+R_{\mu}, \quad \sigma_{\nu \nu}=0 .
$$

This extreme non-additivity, in which there is interaction only between different species, favors demixing. The scheme to construct a FMT DF for this model is the same as for the AOV model [99]; the only thing that needs to be changed is $\phi_{0}\left(\eta_{1}, \ldots, \eta_{m}\right)$. This function is determined from the grand partition function of a 0D cavity,

$$
\Xi_{0}=1-m+\sum_{\nu=1}^{m} e^{z_{\nu}} .
$$

Unfortunately, an analytic expression for $\phi_{0}$ cannot be obtained explicitly, so it has to be solved numerically using the relationships

$$
\eta_{\nu}=\frac{z_{\nu} e^{z_{\nu}}}{\Xi_{0}}, \quad \nu=1, \ldots, m
$$

The dimensional crossover behavior of this DF is similar to that of the AOV DF described above, so it shares the same fundamental flaw in 1D. On the other hand, the bulk fluid has no other thermodynamic stable phases than uniform ones (either mixed or demixed): because there is no excluded volume between particles of the same species, no solid phase is stable at high densities. Only when the number of components is large can the solid be more stable than the demixed fluid phases (notice that increasing the number of species increases the stability of the mixed phase, as the probability for a particle to have a neighbor of its same species decreases). There is evidence of this fact, when the number of components $m>31$, coming from a different model: the parallel hard hypercubic model [100]. 


\subsubsection{General Non-additive Mixtures}

Schmidt has recently proposed a FMT for rather general HS binary mixtures [101]. The shape of his DF is

$$
\beta \mathcal{F}_{\text {ex }}\left[\rho_{1}, \rho_{2}\right]=\int \mathrm{d} \boldsymbol{r} \mathrm{d} \boldsymbol{r}^{\prime} \sum_{i j=0}^{3} K_{i j}\left(\left|\boldsymbol{r}-\boldsymbol{r}^{\prime}\right|\right) \Phi_{i j}\left(\left\{n_{\nu}^{(k)}\right\},\left\{n_{\mu}^{(l)}\right\}\right),
$$

where the $\Phi_{i j}$ are functions of weighted densities, as usual in FMT. Two novelties render this functional peculiar: first of all, the weights are all scalar, as in Kierlik and Rosinberg's version of FMT [56, 57] (using derivatives of delta functions instead of vector and tensor weights); secondly, there is a kernel $K_{i j}\left(\left|\boldsymbol{r}-\boldsymbol{r}^{\prime}\right|\right)$ which accounts for the non-additivity. The components of this kernel are also weights á la Kierlik-Rosinberg (with higher order derivatives), but their radius is $R_{12}$ instead of the radii of the spheres. Both the kernel and the weights are tailored so as to recover the lowest order of the density expansion of $\beta \mathcal{F}_{\text {ex }}$.

At low densities of both components, the structure of the fluid is satisfactorily reproduced, something to be expected because it is in the very construction of FMT. The prediction of the demixing transition is also quite accurate compared to simulations. However, this functional has two serious drawbacks. The first one is that in the limit $\Delta_{12} \rightarrow-1$ (so that $R_{12}=0$ and the two species do not see each other), the functional is not the sum of two DF, one for each species, as it should. The second one is that the choice of weights carries the same flaw for the solid phase as the original Rosenfeld's functional, so it can only be used to study density profiles close to a wall and similar problems. But in spite of these problems, the idea of introducing a kernel depending on $R_{12}$ is worth exploring and might be the clue to construct the definitive FMT able to deal with such complex models.

\subsection{Density Functionals for Anisotropic Bodies}

This section is devoted to give a view on different approximations commonly used to construct DFs of fluids composed of anisotropic particles. These particles, which can be molecules or colloidal particles, have, apart from positional, orientational degrees of freedom. They can have prolate (rod-like) or oblate (disk-like) shapes and, because of that, form a set of new phases. The isotropic fluid is the most disordered phase that can be conceived, without positional and orientational order. A direct consequence of the particle anisotropy is the appearance of orientational order where at least one of the principal axes of particles are on average aligned along a preferential direction while the positional order is absent. This phase was discovered in 1888 by the Austrian botanic Friedrich Reinitzer and later classified by Friedel as the nematic phase [102]. The coupling between positional and orientational degrees 
of freedoms makes these fluids to exhibit also, under certain conditions, a phase transition to the so-called smectic phase [102]. The latter is a periodically layered phase composed of prolate particles aligned perpendicular to the layers. In the layer planes, the centers of mass are randomly positioned, which constitutes the main reason why one can think of smectics as a 1D periodic fluids. Some fluids composed of particles with disk-like symmetries exhibit a transition to a columnar phase, in which the centers of mass are randomly positioned in liquid-like columns. The columns are located at the nodes of a 2D grid, while the particle axes are oriented parallel to the columns. One of the crystalline phases present in liquid crystals is the plastic solid, characterized by long-ranged 3D positional order while the orientational order is absent. Although we will not mention other liquid crystalline phases, the number of them found experimentally is much greater than the few examples presented here.

As was already pointed out at the beginning of this chapter, the DF formalism describes any fluid in terms of its density distribution function. The complete set of variables necessary to describe the degrees of freedom of the anisotropic particle is now $\boldsymbol{x} \equiv\{\boldsymbol{r}, \hat{\boldsymbol{\Omega}}\}$, where $\hat{\boldsymbol{\Omega}} \equiv\{\phi, \theta, \chi\}$ is the set of Euler angles that fix the particle orientation. Thus, the density distribution function $\rho(\boldsymbol{x})$ depends, in general, on six variables that makes the study of these systems much more difficult.

All DFs used in the study of liquid crystals belong to one of the two following sets: (i) those that make emphasis on the attractive contribution of the anisotropic pair potential and (ii) those that describe the fluids in terms of their purely repulsive (and usually hard core) interactions. The use of a simple hard core potential to model a real liquid crystal molecule is, of course, a crude simplification, but as it was shown firstly by Onsager $[103,104]$ and further by Frenkel $[105,106,107]$, this simple interaction is enough to stabilize the nematic, smectic, columnar and different crystalline phases. These works showed that the main liquid crystal symmetries can be explained by the entropic nature of the hard core potentials.

As we will see below, many of these functionals have as an important ingredient the thermodynamic (the equation of state) and structural (the direct correlation function) functions taken from the HS fluid. The reason behind this is the lack of good approximations for these functions for fluids composed of anisotropic particles. In the following section, we will describe the DFs mainly based on the attractive anisotropic part of the pair interaction and further, in Sect. 7.10.2, we will present DFs based on purely hard core interactions.

\subsubsection{Mean Field and Related Density Functionals}

The bulk and interfacial phase behavior of liquid crystals has been crucial on determining the origin of several DF approximations. In this section, we will describe their evolution for the particular case of fluids interacting through 
anisotropic attractive pair potentials. At the same time, the main features of liquid crystal phase behavior will be briefly introduced.

One of the first approaches used to describe the liquid crystal isotropicnematic phase transition is due to Maier and Saupe [108, 109, 110]. Their approximation is equivalent to using the most simple DF which includes, apart from the ideal gas term, $\beta \mathcal{F}_{\text {id }}[\rho]=\int d \boldsymbol{x} \rho(\boldsymbol{x})[\ln \rho(\boldsymbol{x})-1]$ (note that $\boldsymbol{x}$ represents the set of spatial and angular variables), an excess part which is approximated in a mean field way with the following attractive pair potential between particles with axial symmetry: $V\left(r, \theta_{12}\right)=-V_{0}(r) P_{2}\left(\cos \theta_{12}\right)$, where $\theta_{12}$ is the angle between the principal axes of the molecules and $P_{2}(x)$ is the second-order Legendre polynomial [111]. The factor $V_{0}(r)>0$ constitutes the spatial dependence of the pair potential, which is assumed to be decoupled from the angular one. This potential, for a fixed $r$, reaches its minimum value for parallel configuration of particles, favoring in such way the nematic order, while is positive for the mutual perpendicular configuration. The choice of this potential, apart from its simplicity, is motivated by the fact that the adequate order parameter that describes the degree of nematic order is just $S \equiv\left\langle P_{2}(\cos \theta)\right\rangle$, where $\theta$ is the angle between the particle axes and the so-called nematic director (the direction of preferential alignment), and the average is taken over the orientational distribution function $h(\theta)=h(\pi-\theta)$, with head-tail symmetry.

The excess part of the free energy functional has the standard mean field form

$$
\mathcal{F}_{\mathrm{ex}}[\rho]=-\frac{1}{2} \int \mathrm{d} \boldsymbol{x}_{1} \int \mathrm{d} \boldsymbol{x}_{2} \rho\left(\boldsymbol{x}_{1}\right) \rho\left(\boldsymbol{x}_{2}\right) V_{0}\left(r_{12}\right) P_{2}\left(\cos \theta_{12}\right)
$$

Due to the absence of positional ordering in the nematic phase, the density distribution function can be expressed as $\rho(\boldsymbol{x})=\rho h(\theta) /(4 \pi)$, where $\rho$ is the fluid density and the angular density function is normalized as $\int_{-1}^{1} \mathrm{~d}(\cos \theta) h(\theta)=1$. The equation (7.141) can be drastically simplified by taking into account that $\hat{\boldsymbol{\Omega}}_{i}=\left\{\theta_{i}, \phi_{i}\right\}$ (the polar and azimuthal angles of the principal axes are sufficient to describe the orientation of axially symmetric bodies) and carrying out the integration over the angles $\phi_{i}$ with the use of the identity [111] $\int \mathrm{d} \phi_{1} \int \mathrm{d} \phi_{2} P_{2}\left(\cos \theta_{12}\right)=(2 \pi)^{2} P_{2}\left(\cos \theta_{1}\right) P_{2}\left(\cos \theta_{2}\right)\left(\theta_{i}\right.$ being the angle between the axis of particle $i$ and the nematic director). The ideal part of the free energy density, $\beta \mathcal{F}_{\mathrm{id}}[\rho] / V \equiv \Phi_{\mathrm{id}}[h(\theta)]$, is a functional of $h(\theta)$ and can be written as $\Phi_{\mathrm{id}}[h(\theta)]=\rho\left[\ln \rho-1+\int_{-1}^{1} d(\cos \theta) h(\theta) \ln h(\theta)\right]$, where the second term is the orientational entropy.

The constrained functional minimization of $\Phi[h(\theta)]=\beta \mathcal{F}[\rho] / V$ with respect to $h(\theta)$ results in

$$
h(\theta)=\frac{\exp \left[a S P_{2}(\cos \theta)\right]}{\int_{-1}^{1} \mathrm{~d}(\cos \theta) \exp \left[a S P_{2}(\cos \theta)\right]},
$$


with $a=\beta v_{0} \rho$ and $v_{0}=4 \pi \int_{0}^{\infty} \mathrm{d} r r^{2} V_{0}(r)$. Multiplying $(7.142)$ by $P_{2}(\cos \theta)$ and integrating over $\cos \theta$ between -1 and 1 , we obtain a self-consistent integral equation to calculate the equilibrium value of $S$ for a fixed temperature $\beta$ and density $\rho$. The insertion of the found equilibrium orientational distribution $h_{\mathrm{eq}}(\theta)$ in the free energy density and the application of the usual double tangent construction allow us to find the coexisting densities for the isotropic and nematic phases at fixed temperature and to calculate the phase diagram of the Maier-Saupe model.

In order to consider within the same model the smectic phase, we need to impose that $\rho(\boldsymbol{x}, \theta)=\rho(z, \theta)$, i.e. the spatial symmetry is broken in the direction of preferential alignment and the density profile is a periodic function with period $d$. In principle, its value should be found through the DF minimization, but as the Maier-Saupe functional does not include a repulsive contribution from the particle core, its absolute minimum is reached at $d=0$, i.e. the density of aligned molecules builds up in and infinitely small region. Thus, the parameter $d$ should be fixed at some value comparable with the molecule length. The constrained functional minimization (with the constraint $d^{-1} \int_{0}^{d} \mathrm{~d} z \int_{-1}^{1} \mathrm{~d}(\cos \theta) \rho(z, \theta)=\rho, \rho$ being the mean density) leads to the following self-consistent integral equation for the density profile

$$
\rho(z, \theta)=\frac{\rho \exp \left[-V_{\text {eff }}(z, \theta)\right]}{d^{-1} \int_{0}^{d} \mathrm{~d} z \int_{-1}^{1} \mathrm{~d}(\cos \theta) \exp \left[-V_{\text {eff }}(z, \theta)\right]},
$$

where the effective potential in reduced thermal units is a functional of the density profile

$$
V_{\text {eff }}(z, \theta)=-\beta\left[\int_{-\infty}^{\infty} \mathrm{d} z^{\prime} \int_{-1}^{1} \mathrm{~d}(\cos \theta) V_{0}\left(z-z^{\prime}\right) P_{2}\left(\cos \theta^{\prime}\right) \rho\left(z^{\prime}, \theta^{\prime}\right)\right] P_{2}(\cos \theta)
$$

with $V_{0}(z)=2 \pi \int_{|z|}^{\infty} \mathrm{d} r r V_{0}(r)$. In principle, this equation can be solved iteratively for a periodic function $\rho(z+d, \theta)=\rho(z, \theta)$ at each point $\left(z_{i}, \cos \theta_{i}\right)$ of the constructed rectangular grid $[0, d] \times[0,1]$.

The phase diagram in the coordinates $T$ and $d$ (conveniently scaled with the attractive potential parameters) qualitatively coincides with the experimental results. For small molecule lengths, the system goes from the isotropic to the nematic and then to the smectic phases as the temperature decreases; for higher values of $d$, there is a direct isotropic to smectic transition $[112,113]$.

Selinger and Nelson applied the same model to study the vapor-fluid interface. For this purpose, they modeled the interface through an external 
potential of the form $V_{\text {surf }}(z, \cos \theta)=-\epsilon_{s} \exp \left[-\left(z / r_{0}\right)^{2}\right] P_{2}(\cos \theta)$ favoring perpendicular alignment of molecules at the interface. They found complete wetting by the nematic phase as the temperature approaches the isotropicnematic coexistence temperature from the isotropic side. They also found incomplete wetting by the smectic phase with one and two layering transitions at temperatures slightly higher than the isotropic-smectic coexistence temperature [113].

As it was mentioned above, the absence of repulsive interactions makes the model non-predictive with respect to the equilibrium value of the smectic period. For the same reason, the model does not exhibit a vapor-liquid phase transition, thus the necessity to model the interface by an external potential. The first DF which included repulsive interactions between particles was developed by Telo da Gama $[114,115]$. The first proposed version was

$$
\mathcal{F}[\rho]=\mathcal{F}_{\mathrm{id}}[\rho]+\mathcal{F}_{\mathrm{HS}}[\rho]+\frac{1}{2} \int \mathrm{d} \boldsymbol{x}_{1} \mathrm{~d} \boldsymbol{x}_{2} \rho\left(\boldsymbol{x}_{1}\right) \rho\left(\boldsymbol{x}_{2}\right) V_{\text {atr }}\left(\boldsymbol{x}_{1}, \boldsymbol{x}_{2}\right),
$$

where the repulsive part was approximated by the HS potential and its contribution was calculated using the LDA (see Sect. 2.4), i.e.

$$
\mathcal{F}_{\mathrm{HS}}[\rho]=\int \mathrm{d} \boldsymbol{r} \Phi_{\mathrm{HS}}(\rho(\boldsymbol{r})),
$$

where $\Phi_{\mathrm{HS}}(\rho)$ is the HS free energy density derived from the compressibility equation of state. With respect to the attractive part, the following approximation was used in $[114,115]$

$$
V_{\text {atr }}\left(\boldsymbol{x}_{1}, \boldsymbol{x}_{2}\right)=V_{0}\left(r_{12}\right)+V_{1}\left(r_{12}\right) P_{2}\left(\theta_{12}\right),
$$

with $V_{i}(r)=0$ for $r<\sigma$ (the HS diameter) while $V_{i}(r)=-\epsilon_{i}\left(r_{0} / r\right)^{6}$ for $r>\sigma, i=0,1$. This potential can be regarded as the first terms in a general spherical harmonic expansion of the full anisotropic potential [116]. The first one, $V_{0}\left(r_{12}\right)$, is the isotropic part responsible, together with the repulsive core, of the vapor-liquid phase separation, while the second term stabilizes the nematic phase if $\epsilon_{1}>0$. It should be noted that (7.145) is nothing but a DF constructed from a perturbative scheme with the HS fluid as the reference system and the attractive contribution as the perturbation. In this scheme, the radial distribution function of the reference fluid is approximated by its low-density limit $g_{\mathrm{ref}}(r ; \eta)=\Theta(r-\sigma)$.

The temperature-density bulk phase diagram predicted by the present model includes a vapor (V)-isotropic liquid (I) coexistence ending in a critical point and a vapor-nematic $(\mathrm{N})$ or I-N coexistence for temperatures below or above the $\mathrm{V}-\mathrm{I}-\mathrm{N}$ triple point; within this functional different interfaces, as the $\mathrm{V}-\mathrm{I}, \mathrm{V}-\mathrm{N}$ and $\mathrm{I}-\mathrm{N}$ ones can be calculated self-consistently in the neighborhood of the triple point. The numerical calculations of the density and order parameter profiles at the $\mathrm{V}-\mathrm{N}$ interface for some fixed value of $\epsilon_{1}$ (selected so as to model the real liquid crystal anisotropy) give complete wetting by the 
I phase (i.e. a surface-induced disordering transition) at the triple point. As explained in Sect. 7.4.1, the surface tension is calculated through

$$
\gamma=\frac{\Omega+p V}{A}
$$

with $\Omega[\rho]$ the equilibrium value of the grand potential, obtained from its minimization with respect to the density $\rho(z)$ and the angular distribution function $h(z, \theta)$ profiles $[\rho(z, \theta)=\rho(z) h(z, \theta)], p$ the bulk pressure at coexistence, $V$ the total system volume and $A$ the surface area of the planar interface. The functional minimization with respect to $h(z, \theta)$ is a constrained minimization with a Lagrange multiplier required to fulfill the condition $\int \mathrm{d}(\cos \theta) h(z, \theta)=1$. The nematic order parameter profile, calculated as $S(z)=\int \mathrm{d}(\cos \theta) h(z, \theta) P_{2}(\cos \theta)$, is a function of the interface coordinate. To implement the numerical minimization, the density and order parameter are fixed at the left and right sides of the minimization box and are set to the bulk coexisting values. The box length should be large enough for the full interface to fit in. A pair of coupled integral equations for $\{\rho(z), S(z)\}$ is obtained after the exponentiation of the functional minimization of $\Omega[\rho]$ with respect to $\rho(z)$ and $h(z, \theta)$, the latter multiplied by $P_{2}(\cos \theta)$ and integrated over $\cos \theta$. These equations can be solved iteratively selecting adequate initial guesses for these functions.

The structure of the $\mathrm{V}-\mathrm{N}$ and $\mathrm{I}-\mathrm{N}$ interfaces resulting from this model has monotonic density and order parameter profiles. Also, there is no orientational order at the $\mathrm{V}-\mathrm{I}$ interface (the order parameter is always zero along the interface). Finally, the surface tension does not depend on the orientation of the nematic director through the interface. All these results are in contradiction with experiments on liquid crystals which usually find an excess surface ordering near the interface with particles aligned in preferential directions with respect to it. Besides, the density and order parameter profiles have an oscillating behavior near the interface. All these features can be obtained by improving the model with (i) the use of a non-local DF approximation to evaluate the free energy of the HS reference fluid, which makes the density profile to be an oscillating function around the interface position and (ii) the inclusion of higher order terms in the spherical harmonic expansion of the attractive potential; specially, those terms which couple the positional and orientational degree of freedoms of particles (those proportional to $P_{2}\left(\cos \theta_{i}^{\prime}\right)$, with $\theta_{i}^{\prime}$ being the angle between the axis of particle $i=1,2$ and the radius vector joining the centers of mass of particles 1 and 2). The inclusion of these terms can favor a perpendicular alignment of particles at the interface. They also contribute to generate an excess of nematic ordering in regions of the space where the density gradient is large. Moreover, as we will see shortly, the presence of these terms are necessary to stabilize the smectic phase.

All these requirements were included in the DF version for smectic liquid crystals developed by Mederos and Sullivan [117]. As the reference part of the free energy functional, they took a parallel hard ellipsoid (PHE) fluid whose 
functional form is exactly the same as that of a HS fluid with the appropriate scaling along the $z$ direction to transform the HS into ellipsoids of whatever axis ratio, i.e.

$$
\Phi_{\mathrm{PHE}}[\rho(\mathbf{r})]=\rho(\boldsymbol{r}) \psi_{\mathrm{HS}}(\bar{\rho}(\boldsymbol{r})),
$$

where the CS-WDA was used to evaluate the free energy per particle. The weighted density is calculated through

$$
\bar{\rho}(\boldsymbol{r})=\int \mathrm{d} \boldsymbol{r}^{\prime} w\left(\boldsymbol{r}^{\prime} ; \bar{\rho}(\boldsymbol{r})\right) \rho\left(\boldsymbol{r}+\hat{\sigma} \boldsymbol{r}^{\prime}\right),
$$

with $w\left(\boldsymbol{r}^{\prime} ; \bar{\rho}(\boldsymbol{r})\right)$ given by (7.28), (7.32), (7.34) and (7.38). The scaling is accounted for the diagonal tensor $\hat{\sigma}$, with components $\sigma_{\perp}$ along the $x$ and $y$ directions and $\sigma_{\|}$along the $z$ direction (the direction of alignment). Sufficiently large particle aspect ratio $\sigma_{\|} / \sigma_{\perp}$ and the use of attractive pair potential,

$$
\begin{aligned}
V\left(\boldsymbol{x}_{1}, \boldsymbol{x}_{2}\right)= & V_{0}\left(r_{12}\right)+V_{1}\left(r_{12}\right) P_{2}\left(\cos \theta_{12}\right)+V_{2}\left(r_{12}\right)\left[P_{2}\left(\cos \theta_{1}^{\prime}\right)\right. \\
& \left.+P_{2}\left(\cos \theta_{2}^{\prime}\right)\right]
\end{aligned}
$$

are necessary to stabilize the smectic phase. Note the presence of the new terms proportional to $P_{2}\left(\cos \theta_{i}^{\prime}\right)$, which couple the positions and orientations of particles. Taking the proper signs, the third term in (7.151) reaches its minimum value for parallel alignment of particles with their axes perpendicular to the joining vector, i.e. the usual configuration for smectics.

In order to numerically minimize the functional (7.145) for the smectic density profiles, it should be noted that the orientational part of the ideal gas contribution,

$$
S_{\text {or }}[h]=-\int_{-1}^{1} \mathrm{~d} \cos \theta h(z, \theta) \ln [h(z, \theta)],
$$

is an universal function of the nematic order parameter $S(z)$. Thus, this function can be calculated for a uniform nematic phase and tabulated for a regular set of points $S_{i} \in[-1 / 2,1]$. Taking into account that the mean field contribution of the free energy is a functional only of $\rho(z)$ and $S(z)$, the grand potential can be minimized with respect to those functions evaluated at the points of the discretized minimization box which contains at least one smectic period. The conjugate gradient algorithm [20] can be used to achieve this minimization. The values of the orientational entropy and its derivatives with respect to $S\left(z_{i}\right)$ necessary to calculate the energy and the gradients can be evaluated using a cubic spline interpolation from the already tabulated set of points.

The authors of [117] calculated the phase diagrams for different attractive potentials. They found apart from the usual V, I and N phases an stable region of the smectic phase for high densities. The smectic can coexist with 
the nematic or with the isotropic liquid, for small or large values of $\epsilon_{2} / \epsilon_{0}$ as compared to $\epsilon_{1} / \epsilon_{0}, \epsilon_{i}$ being the strength of $V_{i}(r)$.

This model has also been applied to the study of the surface ordering at the wall-isotropic fluid interface, where the external potential of type $V_{s}(z)=-\epsilon_{s} P_{2}(\cos \theta) /\left(z_{0}+z\right)^{3}$ was used to model a flat surface which favors the homeotropic anchoring (perpendicular to the wall-particle alignment). Complete and incomplete wetting by the smectic phase was found together with layering transitions as the strength of the external potential is increased [118, 119]. Finally, the thinning transitions in freely suspended smectic films was also studied with this model [120, 121].

The surface ordering and layering transitions was also found as a result of applying this model to the calculation of the $\mathrm{V}-\mathrm{I}$ interface. To illustrate the strong ordering generated at the interface, we have plotted in Fig. 7.14(a)-(b) the density and order parameter profiles resulting from the minimization of the grand potential at the equilibrium $\mathrm{V}-\mathrm{I}$ interface for a fixed set of parameters $\left\{\epsilon_{i}\right\}$ corresponding to a layering phase transition between two and three smectic layers.

We end this subsection with a very successful version of liquid crystal interparticle potential that has been included in the DF formalism via a perturbation theory and has also been applied in MC simulations of liquid crystal molecules. The origin of this potential can be traced back to the original work of Berne and Pechukas [122], who proposed the so-called Gaussian overlap model. In this work, the authors calculated the effective potential between two ellipsoids with a Gaussian repulsive core. The amplitude and the range of the repulsive potential was calculated through the overlap region between two Gaussians representing the mass distribution of two particles separated by a distance $\boldsymbol{r}$ with their uniaxial axes pointing along $\hat{\boldsymbol{\Omega}}_{i}(i=1,2)$. They
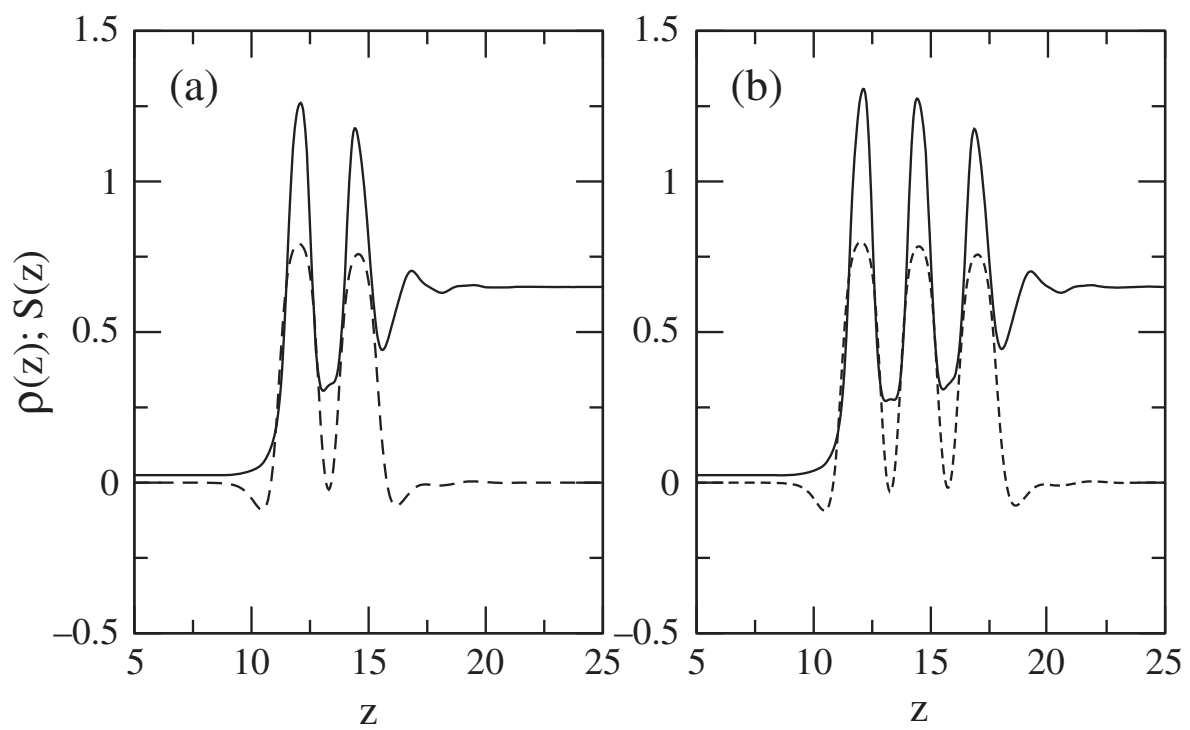

Fig. 7.14. Density $\rho(z)$ (solid lines) and order parameter $S(z)$ (dashed lines) profiles corresponding to the V-I interface containing two (a) and three (b) coexisting smectic peaks as a result of the density functional minimization 
proposed a quite general form for interparticle potentials by the simple rescaling of the interparticle distance by the found range parameter $\sigma\left(\hat{\boldsymbol{\Omega}}, \hat{\boldsymbol{\Omega}}_{1}, \hat{\boldsymbol{\Omega}}_{2}\right)$ given by

$$
\frac{\sigma_{0}^{2}}{\sigma\left(\hat{\mathbf{\Omega}}, \hat{\mathbf{\Omega}}_{1}, \hat{\mathbf{\Omega}}_{2}\right)^{2}}=1-\frac{\chi}{2}\left[\frac{\left(\hat{\mathbf{\Omega}} \cdot \hat{\mathbf{\Omega}}_{1}+\hat{\mathbf{\Omega}} \cdot \hat{\mathbf{\Omega}}_{2}\right)^{2}}{1+\chi \hat{\mathbf{\Omega}}_{1} \cdot \hat{\mathbf{\Omega}}_{2}}+\frac{\left(\hat{\mathbf{\Omega}} \cdot \hat{\mathbf{\Omega}}_{1}-\hat{\mathbf{\Omega}} \cdot \hat{\mathbf{\Omega}}_{2}\right)^{2}}{1-\chi \hat{\mathbf{\Omega}}_{1} \cdot \hat{\mathbf{\Omega}}_{2}}\right]
$$

which explicitly depends on their particle orientations and on the unit vector joining their center of mass $\hat{\boldsymbol{\Omega}}$. The anisotropy parameter $\chi$ is determined by the aspect ratio of the ellipsoidal Gaussians $\kappa=\sigma_{\|} / \sigma_{\perp}$ through $\chi=\left(\kappa^{2}-1\right) /\left(\kappa^{2}+1\right)$. The potential amplitude was later modified from its original form so as to take into account appropriately the anisotropy by including some fitting parameters in its functional form [123]. These parameters were selected in such a way as to quantitatively reproduce the well depths and well widths for the side-by-side and end-to-end particle configurations of the Lennard-Jones (LJ) site potentials (where each particle is formed by $n$ LJ centers positioned along a straight line). The resulting pair potential, known as the Gay-Berne potential [123], was later included in a perturbative scheme of DF formalism [124, 125] to calculate the phase diagram of a liquid crystal model. The resulting temperature-density phase diagram, which includes vapor, isotropic liquid and nematic phases, compares reasonably well with the simulation results $[124,125]$. A further extension of the model was made to include the smectic symmetry. A comparison of the results with the available computer simulations indicates that the theory leads to qualitatively correct predictions $[124,125]$.

\subsubsection{Density Functionals for Hard Anisotropic Particles}

Among hard anisotropic particles, the hard spherocylinder (HSC) is the paradigmatic hard body on which most theoretical and simulation studies have been done. It consists of a cylinder of length $L$ and diameter $D$ capped by two hemispheres of the same diameter. The reason for this particular choice is the simplicity to implement the HSC pair overlap criterion in simulations and also the simple analytic expression that the HSC excluded volume has. The excluded volume between two hard bodies is defined as the spatial region excluded to the center of mass of a second particle, for fixed orientations of both particles, and has for HSCs the following form

$$
V_{\text {excl }}\left(\boldsymbol{\Omega}_{1}, \boldsymbol{\Omega}_{2}\right)=2 L^{2} D|\sin \gamma|+2 \pi D^{2} L+\frac{4}{3} \pi D^{3},
$$

with $\gamma$ the relative angle between the long axes of both particles. 


\section{Density Functionals for Uniform Fluid Phases}

For uniform fluids (as the isotropic or nematic phases), the virial expansion of the excess part of the free energy density up to third order reads

$$
\begin{aligned}
\Phi_{\mathrm{ex}}[h(\hat{\boldsymbol{\Omega}})]= & B_{2}[h(\hat{\boldsymbol{\Omega}})] \rho^{2}+\frac{1}{2} B_{3}[h(\hat{\boldsymbol{\Omega}})] \rho^{3}+\cdots, \\
B_{2}[h(\hat{\boldsymbol{\Omega}})]= & \frac{1}{2} \int \mathrm{d} \hat{\Omega}_{1} \int \mathrm{d} \hat{\Omega}_{2} h\left(\hat{\boldsymbol{\Omega}}_{1}\right) h\left(\hat{\boldsymbol{\Omega}}_{2}\right) V_{\mathrm{excl}} \hat{\boldsymbol{\Omega}}_{1}, \hat{\boldsymbol{\Omega}}_{2}, \\
B_{3}[h(\hat{\boldsymbol{\Omega}})]= & \frac{1}{3 V}\left[\prod_{i=1}^{3} \int d \boldsymbol{x}_{i} h\left(\hat{\boldsymbol{\Omega}}_{i}\right)\right] f\left(\boldsymbol{r}_{12}, \hat{\boldsymbol{\Omega}}_{1}, \hat{\boldsymbol{\Omega}}_{2}\right) \\
& \times f\left(\boldsymbol{r}_{23}, \hat{\boldsymbol{\Omega}}_{2}, \hat{\boldsymbol{\Omega}}_{3}\right) f\left(\boldsymbol{r}_{13}, \hat{\boldsymbol{\Omega}}_{1}, \hat{\boldsymbol{\Omega}}_{3}\right),
\end{aligned}
$$

where $V$ is the system volume and $f\left(\boldsymbol{r}_{i j}, \hat{\boldsymbol{\Omega}}_{i}, \hat{\boldsymbol{\Omega}}_{j}\right)$ is the Mayer function between two particles with fixed orientations $\hat{\boldsymbol{\Omega}}_{i}$ and $\hat{\boldsymbol{\Omega}}_{j}$. Onsager showed that for the isotropic fluid $[h(\hat{\boldsymbol{\Omega}})=1 /(4 \pi)]$, the following asymptotic behavior is fulfilled for large aspect ratios $\kappa \equiv(L+D) / D: B_{3}^{\text {iso }} /\left(B_{2}^{\text {iso }}\right)^{2} \sim \kappa^{-1} \ln \kappa[103,104]$. Then, after scaling the density $\rho$ with $B_{2}^{\text {iso }}=\frac{\pi}{4} D L^{2}$ to define the dimensionless density $\rho^{*}=\rho B_{2}^{\text {iso }}$, the excess free energy in the limit of infinite elongation $\kappa \rightarrow \infty$ retains only the second virial contribution. This approach, extended also to the study of $\mathrm{HSC}$ with a finite aspect ratio $\kappa$, is known as the Onsager approximation.

The constrained functional minimization of the total free energy with respect to $h(\hat{\boldsymbol{\Omega}})$ within the Onsager approach results in an integral equation of the form

$$
\begin{aligned}
h(\theta) & =\frac{\exp \left[-\frac{8 \rho^{*}}{\pi} \int \mathrm{d}\left(\cos \theta^{\prime}\right) K\left(\theta, \theta^{\prime}\right) h(\theta)\right]}{\int_{-1}^{1} \mathrm{~d}(\cos \theta) \exp \left[-\frac{8 \rho^{*}}{\pi} \int \mathrm{d}\left(\cos \theta^{\prime}\right) K\left(\theta, \theta^{\prime}\right) h\left(\theta^{\prime}\right)\right]}, \\
K\left(\theta, \theta^{\prime}\right) & =\int_{0}^{2 \pi} \mathrm{d} \phi \sqrt{1-\left(\cos \theta \cos \theta^{\prime} \cos \phi+\sin \theta \sin \theta^{\prime}\right)^{2}},
\end{aligned}
$$

where the axial symmetry of the HSC has been used $[h(\hat{\boldsymbol{\Omega}})=h(\theta)], \theta$ is the angle between the HSC axis and the nematic director. Equation (7.158) can be solved iteratively for the equilibrium orientational distribution function $h(\theta)$ at a fixed $\rho^{*}$. Then, after the insertion of this function into the freeenergy density and the use of the usual double-tangent construction to find the coexistence densities of the isotropic and nematic phases, the following values are obtained: $\rho_{I}^{*}=3.290$ and $\rho_{N}^{*}=4.191$ while the nematic order parameter at $\rho_{N}^{*}$ is $S=0.7922[126]$. 


\section{The Parsons-Lee Approach}

For finite values of $\kappa$, further virial coefficients should be included if we want to accurately reproduce the $\mathrm{I}-\mathrm{N}$ coexistence densities. The inclusion of the third virial coefficient $B_{3}[h(\theta)]$ in the virial expansion involves the numerical calculation of the integral (7.157), which is not an analytic function of the relative orientations $\hat{\boldsymbol{\Omega}}_{i}$ and of the particle characteristic lengths. Thus, a multidimensional integral must be evaluated, making the functional minimization a numerically difficult task. Parsons and later Lee conceived a DF which includes all the virial coefficients after the third approximately $[127,128,129]$. They proposed as the excess free energy density of the HSC

$$
\Phi_{\mathrm{ex}}[h(\theta)]=\rho \psi_{\mathrm{HS}}(\eta) \frac{B_{2}[h(\theta)]}{B_{2}^{(\mathrm{HS})}},
$$

where $B_{2}^{(\mathrm{HS})}=4 v$ is the HS second virial coefficient, with $v$ the hard rod volume selected equal to the HS volume and $\psi_{\mathrm{HS}}(\eta)=\eta(4-3 \eta) /(1-\eta)^{2}$ is the CS excess free energy per particle of the HS fluid, with $\eta=\rho v$ its packing fraction. This approximation, known as the Parsons-Lee (PL) approximation, recovers the second virial low-density limit while it approximates the remain virial coefficients by the expressions

$$
B_{n}[h(\theta)]=(n-1)(n+2) v^{n-1} \frac{B_{2}[h(\theta)]}{B_{2}^{(H S)}} .
$$

This simple approach gives quite reasonable values for the coexisting densities as compared to the simulation results. For example, for $\kappa=5$, the PL approach gives $\eta_{I}=0.3995$ and $\eta_{N}=0.4172$, while simulations show an isotropic-nematic phase transition at packing fraction equal to 0.4 .

\section{The Scaled Particle Theory}

The SPT, originally developed by Reiss $[23,24]$ for HS and later extended to anisotropic particles by several workers [130, 131, 132, 133, 134], has been successfully applied to the study of liquid crystal models composed of hard convex anisotropic particles. This is a systematically constructed theory which approximates the thermodynamic work to insert a scaled particle with a fixed orientation in a fluid. The approximation amounts to interpolating that work between its two known limits: those corresponding to small and large values of the scaling parameters.

For the scaled HSC with length and width equal to $\lambda_{1} L$ and $\lambda_{2} D$, respectively ( $\lambda_{i}$ are the scaling parameters), in the limit $\lambda_{i} \rightarrow 0$ the probability that the inserted particle does not overlap with any of the fluid particles is $\exp \left[-\beta W\left(\hat{\boldsymbol{\Omega}} ; \lambda_{1}, \lambda_{2}\right)\right],\left(W\left(\hat{\boldsymbol{\Omega}} ; \lambda_{1}, \lambda_{2}\right)\right.$ being the thermodynamic work necessary 
to insert the scaled particle with orientation $\hat{\boldsymbol{\Omega}}$. Taking only into account overlaps of two particles, one finds

$$
\exp \left[-\beta W\left(\hat{\mathbf{\Omega}} ; \lambda_{1}, \lambda_{2}\right)\right]=1-\rho \int \mathrm{d} \hat{\mathbf{\Omega}}^{\prime} h\left(\hat{\mathbf{\Omega}}^{\prime}\right) V_{\operatorname{excl}}\left(\hat{\mathbf{\Omega}}, \hat{\mathbf{\Omega}}^{\prime} ; \lambda_{1}, \lambda_{2}\right)
$$

where $V_{\text {excl }}\left(\hat{\boldsymbol{\Omega}}, \hat{\mathbf{\Omega}}^{\prime} ; \lambda_{1}, \lambda_{2}\right)$ is the excluded volume between the scaled particle and a fluid particle with orientation $\hat{\Omega}^{\prime}$. For large values of $\lambda_{i}$, the work required to insert the particle is just a work required to open a macroscopic cavity of volume equal to that of the scaled particle against the fluid pressure, i.e $W\left(\hat{\boldsymbol{\Omega}} ; \lambda_{1}, \lambda_{2}\right)=p V\left(\lambda_{1}, \lambda_{2}\right)$. In the scaled particle treatment, it is assumed that the work to add a particle with arbitrary values of $\lambda_{i}$ can be calculated from the expansion of $W\left(\hat{\boldsymbol{\Omega}} ; \lambda_{1}, \lambda_{2}\right)$ obtained from Eq. $(7.162)$ by the Taylor series around $(0,0)$ up to second order and adding $p V\left(\lambda_{1}, \lambda_{2}\right)$ as the third-order term. The excess chemical potential of HSPCs with length $L$ and diameter $D$ can be obtained by setting $\lambda_{i}=1$ and integrating over all possible orientations with the distribution function, i.e. $\mu_{\mathrm{ex}}=\int \mathrm{d} \hat{\mathbf{\Omega}} h(\hat{\mathbf{\Omega}}) W(\hat{\mathbf{\Omega}} ; 1,1)$. The pressure can then be obtained through the Gibbs-Duhem equation $\partial p / \partial \rho=1+\rho \partial \mu_{\mathrm{ex}} / \partial \rho$. This yields an excess free energy per particle $\psi[h]=\mu_{\mathrm{ex}}+1-p / \rho$, given by

$$
\begin{aligned}
& \psi[h]=-\ln (1-\eta)+A[h] y+\frac{1}{2} B[h] y^{2}, \\
& A[h]=3\left(1+\frac{4(\kappa-1)^{2}}{\pi(3 \kappa-1)}\langle\langle|\sin \gamma|\rangle\rangle\right), \\
& B[h]=\frac{12 \kappa}{(3 \kappa-1)^{2}}\left(2 \kappa-1+\frac{4}{\pi}(\kappa-1)^{2}\langle\langle|\sin \gamma|\rangle\rangle\right),
\end{aligned}
$$

with $y=\eta /(1-\eta)$ and where $\langle\langle u\rangle\rangle=\int \mathrm{d} \hat{\boldsymbol{\Omega}} \int \mathrm{d} \hat{\boldsymbol{\Omega}}^{\prime} h(\hat{\boldsymbol{\Omega}}) h\left(\hat{\boldsymbol{\Omega}}^{\prime}\right) u\left(\hat{\boldsymbol{\Omega}}, \hat{\boldsymbol{\Omega}}^{\prime}\right)$ defines the double angular average of function $u$ with respect to $h(\hat{\boldsymbol{\Omega}})$. The values that the SPT gives for the I-N coexistence packing fractions for $\kappa=5$ are $\eta_{I}=0.36$ and $\eta_{N}=0.39$, less accurate that the Parson's approach. However, the estimation that the SPT makes for the third virial coefficient is better than Parsons result.

\section{Density Functionals for Non-uniform Liquid Crystal Phases}

All the DFs extended to the study of non-uniform liquid crystal phases (as the smectic phases), except the last versions based on the FMT which will be described in next subsection, include as basic ingredient in their constructions the thermodynamic and structural functions of the HS fluid. See the review of Vroege et al. [135] for a more detailed discussion. Practically, all of them approximate the exact second-order expansion of the excess free energy of the non-uniform fluid around some reference uniform fluid of density $\rho_{0}$ 


$$
\begin{aligned}
\mathcal{F}_{\mathrm{ex}}[\rho(\boldsymbol{x})]= & \mathcal{F}_{\mathrm{ex}}\left[\rho_{0}(\hat{\Omega})\right]+\int \mathrm{d} \boldsymbol{x}_{1} \mu_{\mathrm{ex}}\left[\rho_{0}\left(\hat{\boldsymbol{\Omega}}_{1}\right)\right] \Delta \rho\left(\boldsymbol{x}_{1}\right) \\
& -k T \int \mathrm{d} \boldsymbol{x}_{1} \int \mathrm{d} \boldsymbol{x}_{2} \int_{0}^{1} \mathrm{~d} \lambda \int_{0}^{\lambda} \mathrm{d} \lambda^{\prime} c\left(\boldsymbol{x}_{1}, \boldsymbol{x}_{2} ;\left[\rho_{0}(\hat{\boldsymbol{\Omega}})\right.\right. \\
& \left.\left.+\lambda^{\prime} \Delta \rho(\boldsymbol{x})\right]\right) \Delta \rho\left(\boldsymbol{x}_{1}\right) \Delta \rho\left(\boldsymbol{x}_{2}\right),
\end{aligned}
$$

where $\Delta \rho(\boldsymbol{x})=\rho(\boldsymbol{x})-\rho_{0}(\hat{\boldsymbol{\Omega}})$ and $\rho_{0}(\hat{\boldsymbol{\Omega}})+\lambda^{\prime} \Delta \rho(\boldsymbol{x})$ represents a parameterized integration path from the uniform isotropic or nematic state to the non-uniform final state.

Selecting a reference fluid of zero density and taking the low-density limit of the direct correlation function equal to $f\left(\boldsymbol{x}_{1}, \boldsymbol{x}_{2}\right)$, the Mayer function, we obtain the Onsager approximation extended to the non-uniform fluid

$$
\mathcal{F}_{\text {ex }}[\rho(\boldsymbol{x})]=\frac{1}{2} \int \mathrm{d} \boldsymbol{x}_{1} \int \mathrm{d} \boldsymbol{x}_{2} \rho\left(\boldsymbol{x}_{1}\right) \rho\left(\boldsymbol{x}_{2}\right) f\left(\boldsymbol{x}_{1}, \boldsymbol{x}_{2}\right) .
$$

This approximation has been successfully applied to study the isotropicnematic interface by Shundyak and van Roij, in particular the analysis of particle orientations along the interface [136]. The trivial extension of (7.167) to binary mixtures allowed also the study of the isotropic-nematic and nematicnematic interfaces near an $\mathrm{I}-\mathrm{N}-\mathrm{N}$ triple point that can exhibit certain mixtures of HSCs [137, 138].

\section{The Decoupling Approximation}

Selecting as the reference fluid an isotropic fluid and carrying out a density expansion of the direct correlation function around this reference fluid (7.166) leads to the approximation

$$
\begin{aligned}
\mathcal{F}_{\mathrm{ex}}[\rho(\boldsymbol{x})]= & \mathcal{F}_{\mathrm{ex}}\left[\rho_{0}\right]+\mu_{\mathrm{ex}}\left[\rho_{0}\right] \int \mathrm{d} \boldsymbol{x}_{1} \Delta \rho\left(\boldsymbol{x}_{1}\right) \\
& -\frac{1}{2} k T \int \mathrm{d} \boldsymbol{x}_{1} \int \mathrm{d} \boldsymbol{x}_{2} c\left(\left|\boldsymbol{r}_{1}-\boldsymbol{r}_{2}\right|, \hat{\boldsymbol{\Omega}}, \hat{\boldsymbol{\Omega}}_{1}, \hat{\boldsymbol{\Omega}}_{2} ; \rho_{0}\right) \Delta \rho\left(\boldsymbol{x}_{1}\right) \Delta \rho\left(\boldsymbol{x}_{2}\right)
\end{aligned}
$$

which has been used as the starting point for studies of the smectic [139] and crystalline $[140,141,142]$ phases. However, the function $c\left(\left|\boldsymbol{r}_{1}-\boldsymbol{r}_{2}\right|\right.$, $\left.\hat{\boldsymbol{\Omega}}, \hat{\boldsymbol{\Omega}}_{1}, \hat{\boldsymbol{\Omega}}_{2} ; \rho_{0}\right)$, the direct correlation function for the isotropic fluid of density $\rho_{0}$, is in general unknown (except for the case of HS where we have the PY approximation). Different attempts to approximate this function are all based in the known PY analytic form of the HS direct correlation function. For example, the first recipe was proposed by Pynn, who approximated the direct correlation function of the isotropic HR fluid by the HS PY correlation 
function of the scaled distance $[143,144]$, taking the contact distance between two $\operatorname{HR} \sigma\left(\hat{\mathbf{\Omega}}, \hat{\boldsymbol{\Omega}}_{1}, \hat{\boldsymbol{\Omega}}_{2}\right)$ as the scaling factor, i.e.

$$
c\left(r, \hat{\mathbf{\Omega}}, \hat{\mathbf{\Omega}}_{1}, \hat{\mathbf{\Omega}}_{2} ; \eta\right)=c_{\mathrm{PY}}\left(\frac{r}{\sigma\left(\hat{\mathbf{\Omega}}, \hat{\mathbf{\Omega}}_{1}, \hat{\mathbf{\Omega}}_{2}\right)} ; \eta\right) .
$$

This single anisotropic dependence is known as the decoupling approximation. However, in the limit $r \rightarrow 0$, the correlation function becomes unphysically isotropic. To circumvent this problem, Baus and coworkers $[145,146]$ introduced the following approximation for the hard ellipsoid correlation function

$$
c\left(r, \hat{\mathbf{\Omega}}, \hat{\boldsymbol{\Omega}}_{1}, \hat{\boldsymbol{\Omega}}_{2}, \eta\right)=\frac{V_{\mathrm{excl}}\left(\hat{\boldsymbol{\Omega}}_{1}, \hat{\boldsymbol{\Omega}}_{2}\right)}{v_{0}} c_{\mathrm{PY}}\left(\frac{r}{\sigma_{0}} ; \eta\right),
$$

with $v_{0}=4 \pi \sigma_{0}^{3} / 3$, the excluded HS volume selected to be equal to the ellipsoid volume $\left(\sigma_{0}^{3}=a^{2} b\right)$. Again, the angular and translational dependence are decoupled.

\section{Weighted Density Approximations}

Another route to extend the DF theory to the study of non-uniform phases was developed by Somoza and Tarazona [147, 148, 149] and Poniewierski and Hołyst $[150,151]$. Both approximations are based on the WDA. Both theories introduce the anisotropic correlations through a new weight $w\left(\boldsymbol{r}, \hat{\mathbf{\Omega}}_{1}, \hat{\boldsymbol{\Omega}}_{2}\right)$ which takes care of the orientations of the interacting particles. Somoza and Tarazona selected for the weight the Mayer function $f\left(\boldsymbol{r}, \hat{\boldsymbol{\Omega}}, \hat{\boldsymbol{\Omega}}^{\prime}\right)$ and imposed the requirement the functional recovered the Onsager limit at low densities. Thus, if we define the number of HR which interact with a given particle placed at $\boldsymbol{r}$ and oriented along $\hat{\boldsymbol{\Omega}}$ as

$$
N(\boldsymbol{r}, \hat{\boldsymbol{\Omega}})=\int \mathrm{d} \boldsymbol{r}^{\prime} \int \mathrm{d} \hat{\boldsymbol{\Omega}}^{\prime} \rho\left(\boldsymbol{r}^{\prime}, \hat{\boldsymbol{\Omega}}^{\prime}\right) f\left(\boldsymbol{r}-\boldsymbol{r}^{\prime}, \hat{\boldsymbol{\Omega}}, \hat{\boldsymbol{\Omega}}^{\prime}\right)
$$

and the number of interacting rods in the parallel particle approximation (considering that all of them have the symmetry of an ellipsoid of revolution with the same volume)

$$
N_{\mathrm{PHE}}(\boldsymbol{r})=\int \mathrm{d} \boldsymbol{r}^{\prime} \rho(\boldsymbol{r}) f_{\mathrm{PHE}}\left(\boldsymbol{r}-\boldsymbol{r}^{\prime}\right),
$$

with $\rho(\boldsymbol{r})=\int \mathrm{d} \hat{\boldsymbol{\Omega}} \rho(\boldsymbol{r}, \hat{\boldsymbol{\Omega}})$, the proposed functional is defined by

$$
\mathcal{F}_{\text {ex }}[\rho(\boldsymbol{r}, \hat{\boldsymbol{\Omega}})]=\int \mathrm{d} \boldsymbol{r} \int \mathrm{d} \hat{\boldsymbol{\Omega}} \rho(\boldsymbol{r}, \hat{\boldsymbol{\Omega}}) \psi_{\mathrm{CS}}[\bar{\rho}(\boldsymbol{r})] \frac{N(\boldsymbol{r}, \hat{\boldsymbol{\Omega}})}{N_{\mathrm{PHE}}(\boldsymbol{r})},
$$

where the CS-WDA for a fluid of PHEs was selected to calculate the free energy per particle. Thus, the angular correlations are taken through the 
scaling factor $N(\boldsymbol{r}, \hat{\boldsymbol{\Omega}}) / N_{\mathrm{PHE}}(\boldsymbol{r})$. It should be noted that this approximation recovers Parson's approach for uniform fluids. The aspect ratio of the reference hard ellipsoid was calculated by also requiring that its average tensor of inertia be proportional to that of the HSC.

Hołyst and Poniewierski chose the weight $w\left(\boldsymbol{r}, \hat{\mathbf{\Omega}}, \hat{\mathbf{\Omega}}^{\prime}\right)=f\left(\boldsymbol{r}, \hat{\mathbf{\Omega}}, \hat{\mathbf{\Omega}}^{\prime}\right) /\left(2 B_{2}^{\text {iso }}\right)$ which satisfies the normalization condition $\int \mathrm{d} \boldsymbol{r}\left\langle\left\langle\omega\left(\boldsymbol{r}, \hat{\boldsymbol{\Omega}}, \hat{\boldsymbol{\Omega}}^{\prime}\right)\right\rangle\right\rangle=1$, with the angular brackets meaning the double angular average with respect to the uniform isotropic distribution function $h(\hat{\boldsymbol{\Omega}})=1 /(4 \pi)$ [150, 151]. Thus, the weighted density is calculated through

$$
\bar{\rho}(\boldsymbol{r})=\int \mathrm{d} \boldsymbol{r}^{\prime} \rho\left(\boldsymbol{r}^{\prime}\right)\left\langle\left\langle w\left(\boldsymbol{r}-\boldsymbol{r}^{\prime}, \hat{\mathbf{\Omega}}, \hat{\mathbf{\Omega}}^{\prime}\right)\right\rangle\right\rangle,
$$

where the double angular average is now with respect to the orientational distribution functions $h(\boldsymbol{r}, \hat{\boldsymbol{\Omega}})$ and $h\left(\boldsymbol{r}^{\prime}, \hat{\boldsymbol{\Omega}}^{\prime}\right)$, respectively. The free energy functional in this approximation is calculated as

$$
\beta \mathcal{F}_{\mathrm{ex}}[\rho(\boldsymbol{r}, \hat{\boldsymbol{\Omega}})]=\int \mathrm{d} \boldsymbol{r} \rho(\boldsymbol{r}) \psi(\bar{\rho}(\boldsymbol{r})),
$$

with the excess free energy per particle

$$
\psi(\rho)=\rho B_{2}^{\text {iso }}+\psi_{\mathrm{CS}}(\rho)-4 \eta,
$$

which recovers the exact second virial form for the isotropic fluid.

Phase diagrams of HSC's including isotropic, nematic and smectic phases were calculated using both theories. The nematic-smectic transition was calculated through functional minimization of the Somoza-Tarazona functional and through bifurcation analysis of the Poniewierski-Holyst functional. The transition densities and order parameters of the smectic phase compare reasonably well with simulation results for $\kappa=5$. The Somoza-Tarazona theory predicts a $\mathrm{N}-\mathrm{Sm}$ tricritical point located at $L / D=50$ and a triple $\mathrm{I}-\mathrm{N}-\mathrm{Sm}$ coexistence at $L / D=3$ in agreement with simulations. This model was also successfully implemented in the study of the isotropic-nematic interface of HSCs by Velasco et al. [152].

\subsection{Fundamental Measure Theory for Anisotropic Particles}

The first attempt to extend the FMT to hard convex anisotropic bodies was made by Rosenfeld [153]. He realized that the exact decomposition of the Mayer function between two HS of different radii in terms of the one-particle weights $w_{\mu}^{(\alpha)}(\boldsymbol{r})$ can be regarded as the direct application of the Gauss-Bonnet theorem of differential geometry [154]. This theorem constitutes one of the 
fundamental results in the theory of surfaces and relates the average over a surface of its Gaussian curvature to a topological property of the surface called its Euler characteristic. If a convex body is formed by the intersection volume between two particles $\mu$ and $\nu$, then the Gauss-Bonnet theorem says that Euler characteristics of the surfaces of this intersection volume can be obtained as

$$
4 \pi f_{\mu \nu}(\boldsymbol{r})=\iint_{\partial \mu \cap \nu} K_{\mu} \mathrm{d} S_{\mu}+\iint_{\partial \nu \cap \mu} K_{\nu} \mathrm{d} S_{\nu}+\int_{\partial \mu \cap \partial \nu}\left[k_{g}^{(\mu)}+k_{g}^{(\nu)}\right] \mathrm{d} l,
$$

where $\partial \mu \cap \nu$ is the surface of that part of body $\mu$ which is inside body $\nu$ (the same definition works for $\partial \nu \cap \mu$ ), while $\partial \mu \cap \partial \nu$ is the closed curve obtained by the intersection of both surfaces. $K_{\mu}$ and $K_{\nu}$ are the Gaussian curvatures of surfaces of $\mu$ and $\nu$, while $k_{g}^{(\mu)}$ and $k_{g}^{(\nu)}$ are their geodesic curvatures along their common intersection curve. The first two integrals are surface integrals, while the third one is a line integral. For spheres, it can be shown that

$$
k_{g}^{(\mu)}=\frac{H_{\mu}\left[1-\boldsymbol{u}_{\mu} \cdot \boldsymbol{u}_{\nu}\right]}{\left|\boldsymbol{u}_{\mu} \times \boldsymbol{u}_{\nu}\right|},
$$

with $H_{\mu}$ the mean curvature of the surface of body $\mu$, and $\boldsymbol{u}_{\mu}$ its unit external normal.

If we insert this expression in (7.177), we end up with the decomposition of the Mayer function in terms of a sum of convolutions between one-body weights that defines Rosenfeld's original FMT [13], cf. Eq. (7.65).

The use of (7.178) for any convex body is of course an approximation firstly proposed by Rosenfeld to construct a fundamental measure functional for anisotropic hard bodies. Accordingly, the structure of the functional is exactly the same as his first proposal for HS mixtures (7.113)-(7.115) but with the extended weights

$$
\begin{aligned}
w_{\mu}^{(3)}(\boldsymbol{r}) & =\Theta\left(r-\left|\boldsymbol{R}_{\mu}(\hat{\boldsymbol{\Omega}})\right|\right), & w_{\mu}^{(2)}(\boldsymbol{r}) & =\delta\left(r-\left|\boldsymbol{R}_{\mu}(\hat{\boldsymbol{\Omega}})\right|\right), \\
\boldsymbol{w}_{\mu}^{(2)}(\boldsymbol{r}) & =\boldsymbol{u}_{\mu} \delta\left(r-\left|\boldsymbol{R}_{\mu}(\hat{\boldsymbol{\Omega}})\right|\right), & w_{\mu}^{(0)}(\boldsymbol{r}) & =\frac{K_{\mu}}{4 \pi} w_{\mu}^{(2)}(\boldsymbol{r}), \\
w_{\mu}^{(1)}(\boldsymbol{r}) & =\frac{H_{\mu}}{4 \pi} w^{(2)}(\boldsymbol{r}), & \boldsymbol{w}_{\mu}^{(1)}(\boldsymbol{r}) & =\frac{H_{\mu}}{4 \pi} \boldsymbol{w}_{\mu}^{(2)}(\boldsymbol{r}),
\end{aligned}
$$

where $\boldsymbol{R}_{\mu}(\hat{\boldsymbol{\Omega}})$ is the radius vector from the center of body $\mu$ with orientation $\hat{\Omega}$ to its surface. Once the parametrization of the surface is given, we can calculate the Gaussian $K_{\mu}$ and mean curvature $H_{\mu}$ of body $\mu$ using the standard formulas from differential geometry [154]. The weighted densities are obtained by convoluting the density profiles with (7.179)-(7.181) and integrating the result over $\hat{\Omega}$ 


$$
\begin{aligned}
n^{(\alpha)}(\boldsymbol{r}) & =\sum_{\mu} \int \mathrm{d} \hat{\boldsymbol{\Omega}} \rho_{\mu} * w^{(\alpha)}(\boldsymbol{r}, \hat{\boldsymbol{\Omega}}), \quad \alpha=0,1,2 \\
\boldsymbol{v}^{(\alpha)}(\boldsymbol{r}) & =\sum_{\mu} \int \mathrm{d} \hat{\boldsymbol{\Omega}} \rho_{\mu} * \boldsymbol{w}^{(\alpha)}(\boldsymbol{r}, \hat{\boldsymbol{\Omega}}), \quad \alpha=1,2 \\
\eta(\boldsymbol{r}) & =\sum_{\mu} \int \mathrm{d} \hat{\boldsymbol{\Omega}} \rho_{\mu} * w^{(3)}(\boldsymbol{r}, \hat{\boldsymbol{\Omega}}) .
\end{aligned}
$$

If we impose to the functional that it recovers the DI-FMT form (7.113), (7.114) and (7.120)-(7.121) for the HS limit, we also need to introduce the second-rank tensors $\mathcal{T}_{\mu}(\boldsymbol{r})$ with components $\left(\mathcal{T}_{\mu}\right)_{i j}(\boldsymbol{r})=u_{i}^{(\mu)} u_{i}^{(\mu)} \delta(r-$ $\left.\left|\boldsymbol{R}_{\mu}(\hat{\boldsymbol{\Omega}})\right|\right)$, with $u_{i}^{(\mu)}(i=x, y, z)$ the external unit normals components. With this definition and the Eqs. (7.179)-(7.181), the free energy density obtained from the DI-FMT can in principle be used to obtain a fundamental measure functional for general convex bodies.

This approximation gives the exact second virial coefficient for the isotropic fluid

$$
B_{\mu \nu}^{\text {iso }}=\frac{1}{2}\left[V_{\mu}+A_{\mu} \bar{R}_{\nu}+\bar{R}_{\mu} A_{\nu}+V_{\nu}\right],
$$

with $V_{\mu}, A_{\mu}$ and $\bar{R}_{\mu}=1 /(4 \pi) \int_{\partial \mu} H_{\mu} \mathrm{d} S_{\mu}$, the volume, surface area and mean curvature radius of body $\mu$, respectively. The calculation of the direct correlation function from this generalized functional gives an expression with different terms proportional to $\Delta V_{\mu \nu}, \Delta A_{\mu \nu}$ and $\Delta R_{\mu \nu}$, the volume, the surface area and the mean curvature radius of the intersection of the two bodies, which is consistent with the PY approximation. However, the low density term gives only an approximation for the Mayer function $f_{\mu \nu}$. One can think that this is a minor fault of the present theory, unfortunately, the substitution of the exact Mayer function by the approximated one in terms of a sum of convolutions of single body weights has as a consequence that the Fourier transform of the direct correlation function at the zero wave vector $\boldsymbol{q}=\mathbf{0}$ is always isotropic [155]. This in turn means that the isotropic fluid is always stable with respect to an orientationally ordered phase (as the nematic phase), which is obviously unphysical.

To solve this problem, different approximations for the direct correlation function of anisotropic fluids were worked out by Chamoux and Perera [155]. One of them is obtained from the second functional derivative of the extended Rosenfeld functional replacing its low-density term by another one proportional to the exact Mayer function $f_{\mu \nu}$ [155]. This approximation gives, from the compressibility route $1-\sum_{i j} \rho_{i} \rho_{j} \hat{c}_{i j}(\boldsymbol{q}=\mathbf{0})=\partial(\beta p) / \partial \rho$, the scaled particle equation of state and also exhibits an spinodal isotropic-nematic instability at $\boldsymbol{q}=\mathbf{0}$.

Another framework to construct DFs for anisotropic particles is based on the deconvolution of the exact Mayer function in terms of weights that can 
also depend on the orientations of both particles. If we renounce to have single body weights, then the decomposition can be exactly obtained, but for some special limits of the particle anisotropy. This approximation will be described later.

\subsubsection{FMT for Parallel Hard Cubes}

Beside HS, there is another particle geometry for which a FMT has been consistently developed, namely the case of a fluid of parallel hard cubes $[156,157,158]$. We briefly describe the theory here. The Mayer function between two parallel cubes of edge lengths $\sigma_{\mu}$ and $\sigma_{\nu}$ is

$$
f_{\mu \nu}(\boldsymbol{r})=\Theta\left(\sigma_{\mu \nu}-|x|\right) \Theta\left(\sigma_{\mu \nu}-|y|\right) \Theta\left(\sigma_{\mu \nu}-|z|\right),
$$

with $\sigma_{\mu \nu}=\left(\sigma_{\mu}+\sigma_{\nu}\right) / 2$. This Mayer function can be decomposed exactly in terms of a finite sum of convolutions between one-particle weights as

$$
\begin{aligned}
f_{\mu \nu}(\boldsymbol{r})= & w_{\mu}^{(3)} * w_{\nu}^{(0)}(\boldsymbol{r})+w_{\mu}^{(0)} * w_{\nu}^{(3)}(\boldsymbol{r})+\boldsymbol{w}_{\mu}^{(2)} * \boldsymbol{w}_{\nu}^{(1)}(\boldsymbol{r}) \\
& +\boldsymbol{w}_{\mu}^{(1)} * \boldsymbol{w}_{\nu}^{(2)}(\boldsymbol{r}),
\end{aligned}
$$

with the weights defined as

$$
\begin{aligned}
w_{\mu}^{(0)}(\boldsymbol{r}) \equiv & \zeta_{\mu}(x) \zeta_{\mu}(y) \zeta_{\mu}(z), \\
\boldsymbol{w}_{\mu}^{(1)}(\boldsymbol{r}) \equiv & \left(\tau_{\mu}(x) \zeta_{\mu}(y) \zeta_{\mu}(z), \zeta_{\mu}(x) \tau_{\mu}(y) \zeta_{\mu}(z),\right. \\
& \left.\zeta_{\mu}(x) \zeta_{\mu}(y) \tau_{\mu}(z)\right), \\
\boldsymbol{w}_{\mu}^{(2)}(\boldsymbol{r}) \equiv & \left(\zeta_{\mu}(x) \tau_{\mu}(y) \tau_{\mu}(z), \tau_{\mu}(x) \zeta_{\mu}(y) \tau_{\mu}(z),\right. \\
& \left.\tau_{\mu}(x) \tau_{\mu}(y) \zeta_{\mu}(z)\right) \\
w_{\mu}^{(3)}(\boldsymbol{r}) \equiv & \tau_{\mu}(x) \tau_{\mu}(y) \tau_{\mu}(z),
\end{aligned}
$$

where $\zeta_{\mu}(x)=\frac{1}{2} \delta\left(\frac{\sigma_{\mu}}{2}-|x|\right)$ and $\tau_{\mu}(x)=\Theta\left(\frac{\sigma_{\mu}}{2}-|x|\right)$. Introducing two more weights as $\omega_{\mu}^{(1)}(\boldsymbol{r}) \equiv \boldsymbol{u} \cdot \boldsymbol{\omega}_{\mu}^{(1)}(\boldsymbol{r})$ and $\omega_{\mu}^{(2)}(\boldsymbol{r})=\boldsymbol{u} \cdot \boldsymbol{\omega}_{\mu}^{(2)}(\boldsymbol{r})[\boldsymbol{u}=(1,1,1)]$, the most general form of the excess part of free energy density according to a dimensional analysis is

$$
\begin{aligned}
\Phi= & a_{0} n^{(0)}+a_{1} n^{(1)} n^{(2)}+b_{1} \boldsymbol{v}^{(1)} \cdot \boldsymbol{v}^{(2)}+a_{2}\left(n^{(2)}\right)^{3} \\
& +b_{2} n^{(2)} \boldsymbol{v}^{(2)} \cdot \boldsymbol{v}^{(2)}+c \boldsymbol{v}^{(2)} \cdot \boldsymbol{v}^{(2)} \cdot \boldsymbol{v}^{(2)}
\end{aligned}
$$

where the weighted densities are introduced as usual, $n^{(\alpha)}(\boldsymbol{r})=\sum_{\mu} \rho_{\mu} * w_{\mu}^{(\alpha)}$, $\boldsymbol{v}^{(\alpha)}(\boldsymbol{r})=\sum_{\mu} \rho_{\mu} * \boldsymbol{w}_{\mu}^{(\alpha)}$ and $\boldsymbol{v} \cdot \boldsymbol{v} \cdot \boldsymbol{v} \equiv v_{x}^{3}+v_{y}^{3}+v_{z}^{3}$. The coefficients $a_{i}(\eta)$, $b_{i}(\eta)$ and $c(\eta)$ are all functions of the dimensionless local packing fraction $\eta=\sum_{\mu} \rho_{\mu} * w_{\mu}^{(3)}$. Introducing Eq. (7.192) in the SPT differential equation $[156,157,158]$ 


$$
-\Phi+\sum_{\alpha} m^{(\alpha)} \frac{\partial \Phi}{\partial m^{(\alpha)}}+n^{(0)}=(1-\eta) \frac{\partial \Phi}{\partial \eta}
$$

with $m^{(\alpha)} \equiv\left\{n^{(\alpha)}, \boldsymbol{v}^{(\alpha)}\right\}$, we obtain a set of first-order differential equations to determine $\left\{a_{i}, b_{i}, c\right\}$ up to the integration constants. Note that Eq. (7.193) can be easily obtained from the usual definition of the fluid pressure together with the identity $\beta p=\partial \Phi / \partial \eta$ valid for the SPT. The density expansion of the direct correlation function $c_{i j}(\boldsymbol{r})$ obtained from (7.192) together with the exact knowledge of the Mayer and the triangle diagrams, obtained as sums of convolutions between single particle weights, allow us to fix all the integration constants but one. Finally, imposing the exact dimensional crossover to zero dimension, this constant can be determined, resulting in the functional

$$
\Phi^{(3)}=-n^{(0)} \ln (1-\eta)+\frac{\boldsymbol{v}^{(1)} \cdot \boldsymbol{v}^{(2)}}{1-\eta}+\frac{v^{(2 x)} v^{(2 y)} v^{(2 z)}}{(1-\eta)^{2}} .
$$

It should be noted that the free energy evaluated at density profiles corresponding to arbitrary 0D cavities exactly recovers the $0 \mathrm{D}$ limit, i.e. the parallel hard cubes fluid is free from lost cases. The fundamental reason behind this is that there are no configurations of three particles having pairwise overlap but no triple overlap.

Based on this property, FMT functionals for hard core particles on a lattice were constructed by the projections of the parallel hard hypercube lattice model on different planes. The FMT for lattice model can then be reformulated based on the exact 0D crossover, and functionals for arbitrary hard particles on a lattice can be obtained that are free from lost cases [159, 160, 161, 162, 163, 164]. The same procedure of projecting the hard hypercube geometry on a particular plane was recently implemented to obtain a FMT for hard hexagons in the continuum [165]. It was shown that the number of weighted densities necessary to obtain a functional free from lost cases is infinite. Note that the hexagon geometry allows to have configurations of three particles with pairwise overlap but no triple overlap.

Following the same procedure, we can obtain the 2D functional for a fluid of parallel hard squares as

$$
\Phi^{(2)}=-n^{(0)} \ln (1-\eta)+\frac{v^{(1 x)} v^{(1 y)}}{1-\eta},
$$

where the $2 \mathrm{D}$ weights are

$$
\begin{aligned}
w_{\mu}^{(0)}(\boldsymbol{r}) & \equiv \zeta_{\mu}(x) \zeta_{\nu}(y) \\
\boldsymbol{w}_{\mu}^{(1)}(\boldsymbol{r}) & \equiv\left(\tau_{\mu}(x) \zeta_{\mu}(y), \zeta_{\mu}(x) \tau_{\mu}(y)\right), \\
w_{\mu}^{(2)}(\boldsymbol{r}) & \equiv \tau_{\mu}(x) \tau_{\mu}(y) .
\end{aligned}
$$




\section{One-component Parallel Hard Cube Model}

It is known that the equation of state of the uniform fluid obtained from the FMT is the SPT applied to the parallel hard cubes fluid; the other only stable phase for this model is a cubic crystal, which was calculated in [166]. For this model, the following parametrization of density profile was used

$$
\begin{aligned}
\rho(\boldsymbol{r}) & =\eta_{0}\left(\frac{\alpha}{\pi}\right)^{\frac{3}{2}} \sum_{\boldsymbol{k}} \exp \left[-\alpha\left(\boldsymbol{r}-\boldsymbol{R}_{\boldsymbol{k}}\right)^{2}\right]=\Psi_{0}(x) \Psi_{0}(y) \Psi_{0}(z) \\
\Psi_{0}(x) & =\eta_{0}^{\frac{1}{3}}\left(\frac{\alpha}{\pi}\right)^{\frac{1}{2}} \sum_{k=-\infty}^{\infty} \exp \left[-\alpha(x-k d)^{2}\right]
\end{aligned}
$$

where $\boldsymbol{R}_{\boldsymbol{k}}=\boldsymbol{k} d\left[\boldsymbol{k}=\left(k_{1}, k_{2}, k_{3}\right)\right]$ are the vector positions of the nodes of the simple cubic lattice with period $d$ and $\eta_{0}$ representing the particle occupancy probability per unit cell. With this parametrization, the excess free energy density (7.194) for the one-component parallel hard cube fluid becomes

$$
\Phi^{(3)}=n^{(0)}\left[-\ln (1-\eta)+\frac{3 \eta}{1-\eta}+\frac{\eta^{2}}{(1-\eta)^{2}}\right],
$$

with $n^{(0)}(\boldsymbol{r})=n^{(0)}(x) n^{(0)}(y) n^{(0)}(z), \eta(\boldsymbol{r})=\eta(x) \eta(y) \eta(z)$, where

$$
\begin{aligned}
n^{(0)}(x) & =\frac{1}{2}\left[\Psi_{0}\left(x-\frac{\sigma}{2}\right)+\Psi_{0}\left(x+\frac{\sigma}{2}\right)\right], \\
\eta(x) & =\frac{1}{2}\left[\Psi_{1}\left(x+\frac{\sigma}{2}\right)-\Psi_{1}\left(x-\frac{\sigma}{2}\right)\right], \\
\Psi_{1}(x) & =\eta_{0}^{\frac{1}{3}} \sum_{k} \operatorname{erf}[\sqrt{\alpha}(x+k d)] .
\end{aligned}
$$

Thus, the total free energy per unit volume $\beta \mathcal{F} / V$ is minimized with respect to $\alpha$ and $\eta_{0}$ to find the equilibrium density profile. The mean density $\rho$, the cell period $d$ and the occupancy probability $\eta_{0}$ are related through $\rho=\eta_{0} d^{-3}$. The fluid-solid transition is continuous and occurs at $\eta=0.314$, with a fraction of vacancies of $31 \%$ [166]. The equation of state for the solid phase compares very well with simulation results for mean packing fractions $\eta>0.5$ as can be seen in Fig. 7.15. However, the simulations predict a fluidsolid continuous transition at $\eta \approx 0.48$, higher than the FMT-predicted value of 0.314 [168]. As for HS, the fluid phase is to blame on this discrepancy in the FMT prediction for crystallization. It is known that the SPT equation of state for the fluid of parallel hard cubes deviates from simulation results at intermediate densities $\sim 0.4$. In this sense, the situation is even worse than for the HS fluid because the exact virial expansion has negative coefficients while the expansion of the SPT equation of state has only positive coefficients. 


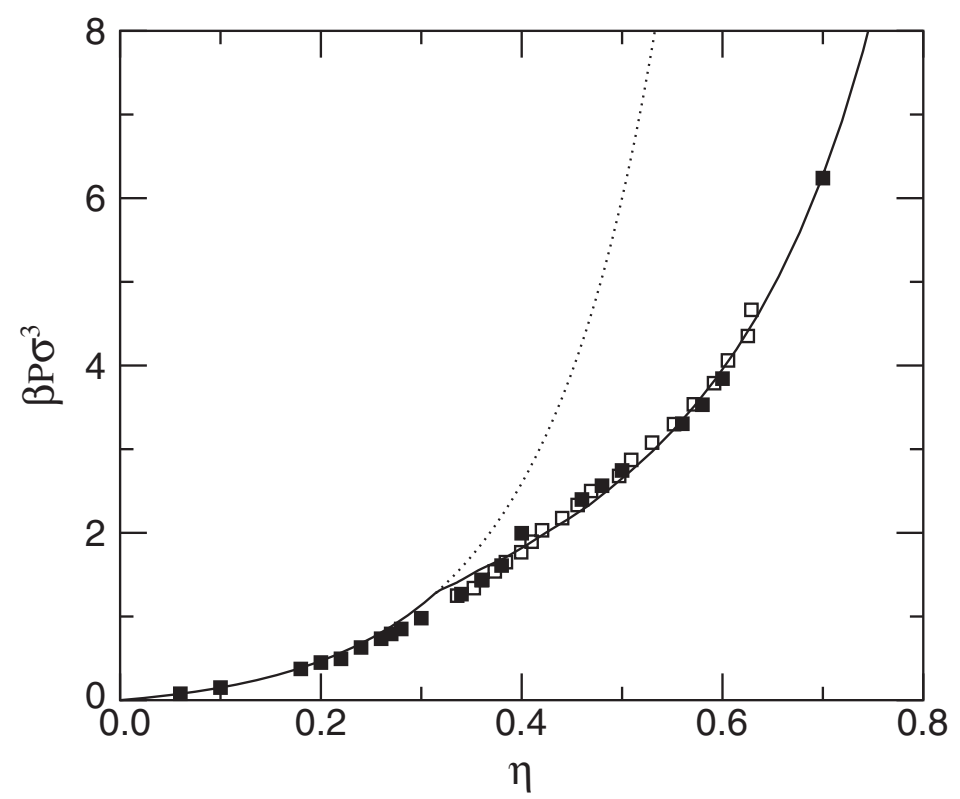

Fig. 7.15. Equation of state of the parallel hard cube system. Solid lines correspond to the pressure of the stable phase (fluid or solid) at a given packing fraction $\eta$. The dotted line is the unstable fluid branch beyond freezing. Full squares are the simulations of [167] and empty squares those of [168]

\section{Binary Mixtures of Parallel Hard Cubes}

The fundamental measure functional defined trough the free energy density (7.194) was used in the study of the demixing transitions in mixtures of parallel hard cubes [166]. Note that again the uniform limit of that functional coincides with the SPT result, and it was shown that for an asymmetry ratio $r \equiv \sigma_{1} / \sigma_{2}>9.98$, the parallel hard cube mixture phase separates in two phases, each one richer in one of the components [166]. In Fig. 7.16(a), we show the fluid-fluid coexistence for different mixtures. The same figure also shows the spinodal curves of the fluid-solid transition. It can be seen in the figure that the fluid-fluid demixing is always preempted by a fluid-solid phase separation. The same analysis for parallel hard square mixtures, using the uniform limit of (7.195), shows that the demixing is forbidden in 2D. However, using the SPT for a mixture of freely rotating hard squares, it can be shown that demixing occurs between an isotropic fluid and a tetratic nematic phase for $r>4$ [169, 170, 171]. The last one, also observed in simulations [172], is an orientationally ordered phase invariant under rotations of $\pi / 2$ (the tetratic symmetry), i.e. in terms of the orientational distribution function, we have $h(\theta)=h(\theta+\pi / 2)$ [169]. In Fig. 7.16 (b), we show the demixing scenario of a mixture of freely rotating hard squares with asymmetry ratio $r=10$ [173]. We can observe the presence of a lower and upper critical points, apart from a tricritical point below which the isotropic-tetratic nematic transition is of second order.

These results show that certain cautions should be taken in the extrapolation of results given from any DF theory of parallel anisotropic particles 

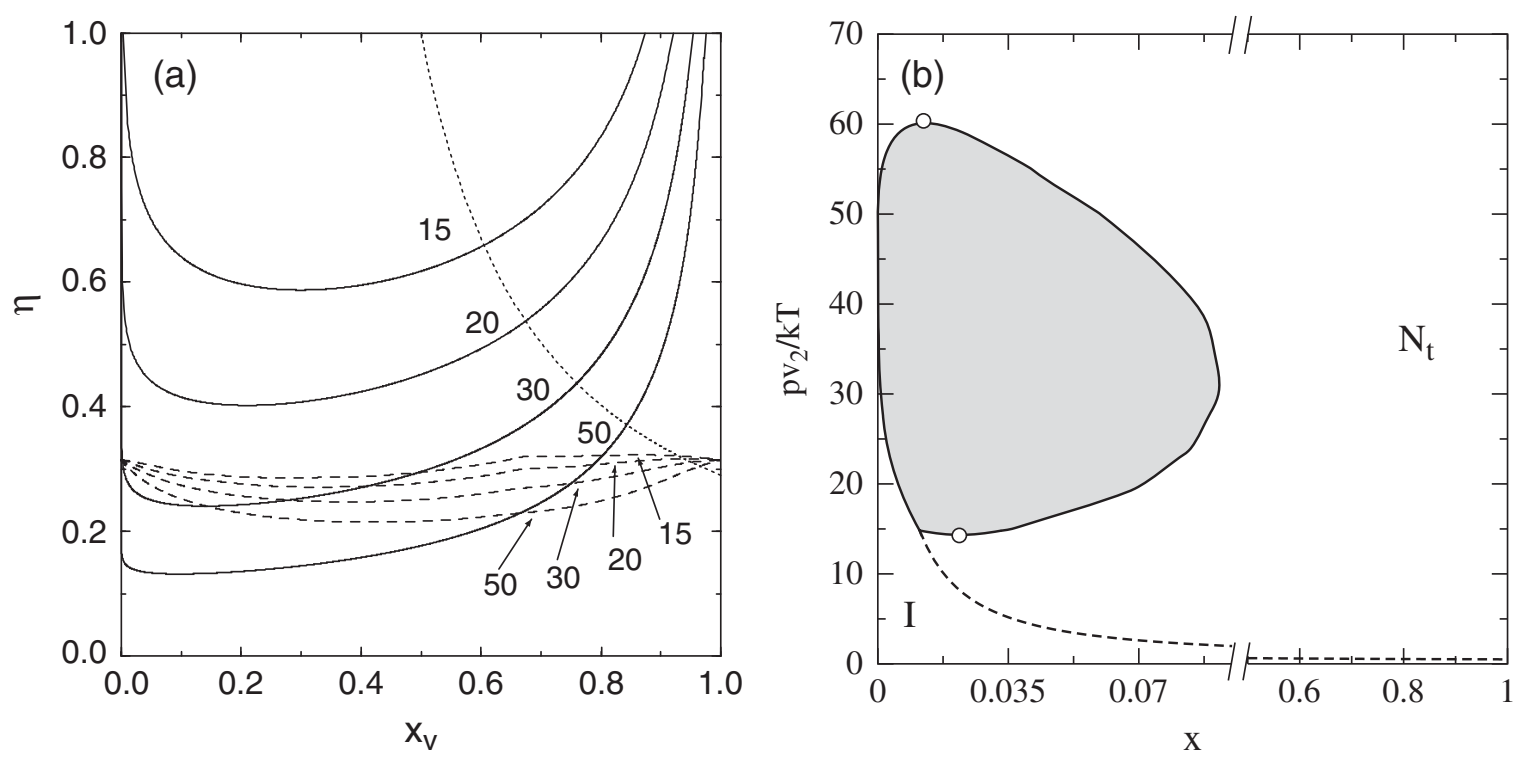

Fig. 7.16. (a): Phase diagram of a binary mixture of parallel hard cubes. Packing fraction $\eta$ versus fraction of volume of big species $x_{v} \equiv \eta_{1} / \eta$. The numbers label the asymmetry ratio $r$. Solid lines show the binodals, while dashed lines represent the fluid-solid spinodals. The positions of critical points are shown with a dotted line. (b): Phase diagram of freely rotating hard squares for $r=10$. Pressure in reduced units versus the molar fraction of big species $x$. Dashed line shows the continuous isotropic-tetratic nematic transition. Open circles show the critical points

to predict the phase behavior of freely rotating particles. The restriction of orientations allows us to obtain a consistent FMT for parallel hard cubes but paying the price of losing information about the orientational ordering phenomena inherent to the particle anisotropy.

\section{The Adhesive Parallel Hard Cube Fluid}

The parallel hard cube model is far from being a realistic model for particle interactions in colloids or in molecular fluids. However, it has been successfully applied to clarify the entropic mechanism behind the fluid-solid demixing that the highly asymmetric binary mixture of hard core particles usually exhibit $[174,175]$. For this purpose, a DF for an adhesive hard cube fluid was obtained taking the limit of infinite asymmetry $\sigma_{2} / \sigma_{1} \rightarrow 0$ of the functional $\Upsilon\left(\mu_{2},\left[\rho_{1}\right]\right)=\mathcal{F}\left[\rho_{1}, \rho_{2}\right]-\mu_{2} \int \mathrm{d} \boldsymbol{r} \rho_{2}(\boldsymbol{r})$ (the thermodynamic potential for the semi-grand ensemble), where 1 and 2 label the solute and solvent particles, respectively. The chemical potential of the smaller component $\mu_{2}$ is fixed to a constant value. As a result, an effective one-component functional for the solute particles is obtained with the following expressions for excess free energy density in dimensions 2 and 3

$$
\Phi_{\mathrm{eff}}^{(D)}=\Phi^{(D)}+\Phi_{\mathrm{ad}}^{(D)}
$$




$$
\begin{aligned}
\Phi_{\mathrm{ad}}^{(2)} & =\frac{z}{8} \frac{\left[g_{1}(z)|\boldsymbol{\nabla} \eta|^{2}-4 g_{2}(z) \boldsymbol{v}^{(1)} \cdot \boldsymbol{v}^{(1)}+8 g_{3}(z) v^{(1 x)} v^{(1 y)}\right]}{1-\eta} \\
\Phi_{\mathrm{ad}}^{(3)} & =\frac{z}{8} \frac{\left[|\boldsymbol{\nabla} \eta|^{2}-4 \boldsymbol{v}^{(2)} \cdot \boldsymbol{v}^{(2)}\right]}{1-\eta}
\end{aligned}
$$

where $g_{1}(z)=1 /(1+z), g_{2}(z)=(1+z) /(1+2 z)$ and $g_{3}(z)=z /(1+2 z)$ are functions of the solvent's fugacity $z=\exp \left(\beta \mu_{2}\right)$. The terms $\Phi_{\text {ad }}^{(D)}$ take into account the residual depletion interaction between solute particles induced by the solvent. The phase diagram of this $3 \mathrm{D}$ adhesive hard cube fluid is shown in Fig. 7.17. The adhesiveness makes the fluid metastable with respect to a phase separation between a close-packed crystal and an infinitely diluted fluid. Different metastable or unstable phases are separated by spinodal lines plotted in the phase diagram (see Fig. 7.17). If a small degree of polydispersity $\Delta_{\sigma} \equiv \sqrt{\left\langle\sigma^{2}\right\rangle /\langle\sigma\rangle^{2}-1}$ is included to avoid the presence of the close-packed solid, then the phase diagram exhibits a fluid-solid or solid-solid coexistence. This result is qualitatively similar to that found in the asymmetric HS binary mixture $[176,177,178,179]$.

\subsubsection{Fundamental Measure Theory for the Zwanzig Model}

It can be shown that the free energy functional for parallel hard hypercube systems in dimension $D$ can be obtained from the $0 \mathrm{D}$ free energy density

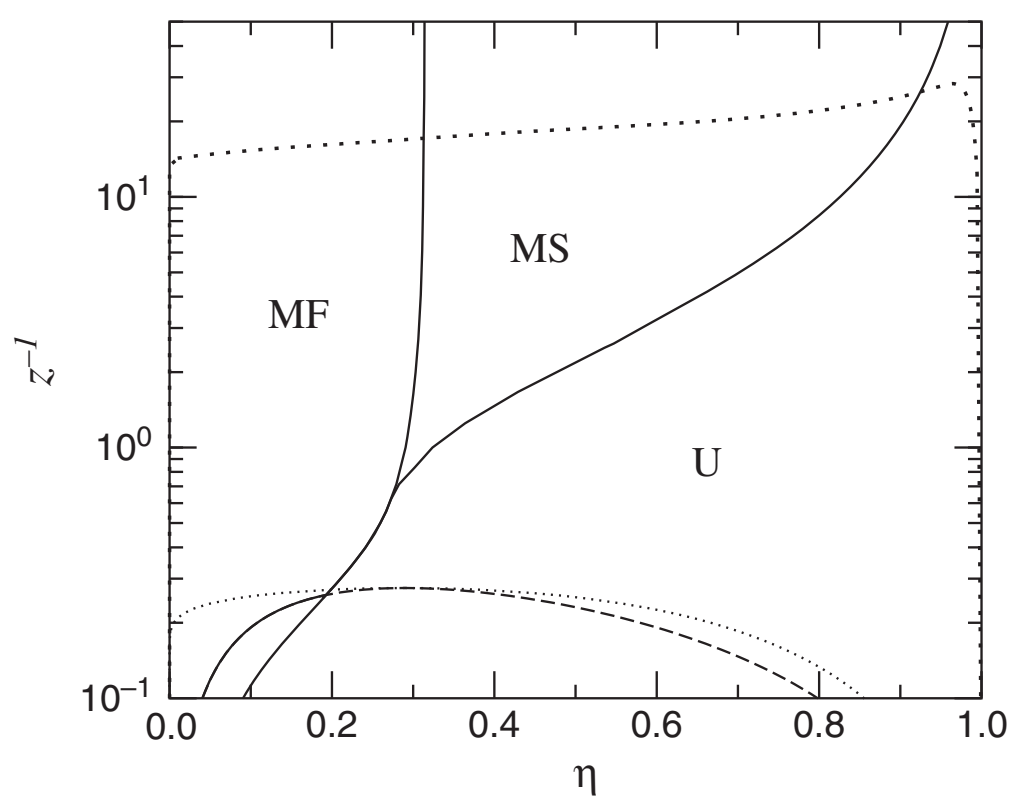

Fig. 7.17. Inverse of the solvent fugacity $z^{-1}$ versus solute packing fraction $\eta$ of the infinitely asymmetric binary mixture of parallel hard cubes. The thick solid line separates the unstable region (U) from the metastable one; the thin one marks the (continuous) transition from a metastable fluid (MF) to a metastable solid (MS); the dashed one is the fluid-fluid spinodal. The dotted lines mark the fluid-solid or solid-solid transition and the fluid-fluid metastable coexistence for a polydisperse fluid with polydispersity $\Delta_{\sigma}=4.5 \%$ 
$\Phi^{(0)}$ by applying a differential operator $[156,157,158]$. Moreover, the same expression can be used to obtain the functional corresponding to a ternary mixture of hard parallelepipeds with restricted orientations (the orientations are restricted to each of the cartesian axes). This result reads

$$
\Phi^{(D)}=\frac{\boldsymbol{\partial}^{D}}{\boldsymbol{\partial} \boldsymbol{\sigma}^{(D)} \cdots \boldsymbol{\partial} \boldsymbol{\sigma}^{(1)}} \Phi^{(0)}(\eta),
$$

where the $D$-dimensional local packing fraction is defined as

$$
\eta(\boldsymbol{r})=\sum_{\mu} \prod_{i=1}^{D}\left(\int_{x_{i}-\sigma_{\mu}^{(i)} / 2}^{x_{i}+\sigma_{\mu}^{(i)} / 2} \mathrm{~d} x_{i}^{\prime}\right) \rho_{\mu}\left(x_{1}^{\prime}, \ldots, x_{D}^{\prime}\right),
$$

and we have introduced the short-hand notation

$$
\frac{\partial}{\partial \boldsymbol{\sigma}^{(i)}} \equiv \sum_{\mu} \frac{\partial}{\partial \sigma_{\mu}^{(i)}}
$$

$\sigma_{\mu}^{(i)}$ being the edge length parallel to the $i$ axis of the species $\mu$ (each species has a different orientation). This result means that we have found a FMT for the model introduced by Zwanzig to study the isotropic-nematic phase transition by restricting the orientations of parallelepipeds to the three cartesian axes $[180,181]$. Through this approximation, the virial coefficients can be calculated up to seventh order. In this way, Zwanzig proved the robust character of the isotropic-nematic phase transition, i.e. that the theoretically predicted transition is not a mere effect of the low-order virial expansion.

\section{Bulk Phase Diagram}

The complete phase diagram of the Zwanzig model, including not only isotropic and nematic phases but also non-uniform phases as smectic, columnar, plastic and oriented solid, has been calculated using FMT [182]. Both, rod and plate symmetries were included in this study. For this purpose, it is better to use a different parametrization of the density profiles which includes all possible symmetries. The truncated Fourier series of the density profile reads

$$
\rho_{\mu}(\boldsymbol{r})=\rho \gamma_{\mu} \sum_{\boldsymbol{k}}^{N} \alpha_{\boldsymbol{k}}^{(\mu)} \prod_{i=1}^{3} \cos \left(\frac{2 \pi}{d_{i}} k_{i} x_{i}\right),
$$

where $\gamma_{\mu}(\mu=x, y, z)$ is the average occupancy probability of the unit cell of species $\mu, \alpha_{\boldsymbol{k}}^{(\mu)}$ are the Fourier amplitudes of the same species and $d_{i}(i=$ $1,2,3)$ are the simple parallelepipedic lattice periods in the $x, y$ and $z$ spatial directions. The cut-off vector $\boldsymbol{N} \equiv\left(N_{1}, N_{2}, N_{3}\right)$ is selected in such a way as 
to guarantee $\alpha_{N}^{(\mu)}<10^{-7}$ for all $\mu$. For uniaxial parallelepipeds, the nematic order parameter $S$ is enough to define all the coefficients $\gamma_{\mu}$, because the symmetry of the problem requires that $\gamma_{\perp}=\gamma_{x}=\gamma_{y}=(1-S) / 3$ and $\gamma_{\|} \equiv \gamma_{z}=(1+2 S) / 3$. The nematic director is set parallel to the $z$ axis. Also, the Fourier amplitude $\alpha_{0,0,0}$ is set to unity. These density profiles should be included in the definitions of the weighted densities $n^{(\alpha)}(\boldsymbol{r})=\sum_{\mu=x, y, z} \rho_{\mu} *$ $\omega_{\mu}^{(\alpha)}(\boldsymbol{r}), \boldsymbol{v}^{(\alpha)}(\boldsymbol{r})=\sum_{\mu=x, y, z} \rho_{\mu} * \boldsymbol{w}_{\mu}^{(\alpha)}(\boldsymbol{r})$ and $\eta(\boldsymbol{r})=\sum_{\mu} \rho_{\mu} * w_{\mu}^{(3)}(\boldsymbol{r})$. The weights are the same functions (7.188)-(7.191) with the functions $\zeta_{\mu}$ and $\tau_{\mu}$ replaced by

$$
\zeta_{\mu}\left(x_{i}\right)=\frac{1}{2} \delta\left(\frac{\sigma_{\mu}^{i}}{2}-\left|x_{i}\right|\right), \quad \tau_{\mu}\left(x_{i}\right)=\Theta\left(\frac{\sigma_{\mu}^{i}}{2}-\left|x_{i}\right|\right),
$$

with $\sigma_{\mu}^{i}=\sigma+(L-\sigma) \delta_{\mu i}$ ( $L$ and $\sigma$ being the length and width of the parallelepiped with $\delta_{\mu i}$ the Kronecker delta). All convolutions defining $n^{(\alpha)}(\boldsymbol{r})$, $\boldsymbol{v}^{(\alpha)}(\boldsymbol{r})$ and $\eta(\boldsymbol{r})$ are trivially computed so they are analytic functions of the minimization variables $S, d_{\perp}, d_{\|}$and $\alpha_{\boldsymbol{k}}^{(\perp, \|)}$. Note that the symmetries of all possible phases are now conveniently included. The smectic, columnar and solid symmetries have vectors $\boldsymbol{k}=\left(0,0, k_{3}\right), \boldsymbol{k}=\left(k_{1}, k_{2}, 0\right)$ and $\boldsymbol{k}=\left(k_{1}, k_{2}, k_{3}\right)$, respectively.

In Figs. 7.18(a) and 7.18(b), we plot, respectively, the phase diagrams for prolate and oblate particles calculated with FMT. Note the presence of the discotic smectic (DS) phase, where the uniaxial particle axes are randomly oriented in the plane of smectic layers. The stability of this peculiar phase is due to the restriction of orientations imposed on the particle axes as was shown by Casey and Harrowell in their MC simulation study of the Zwanzig
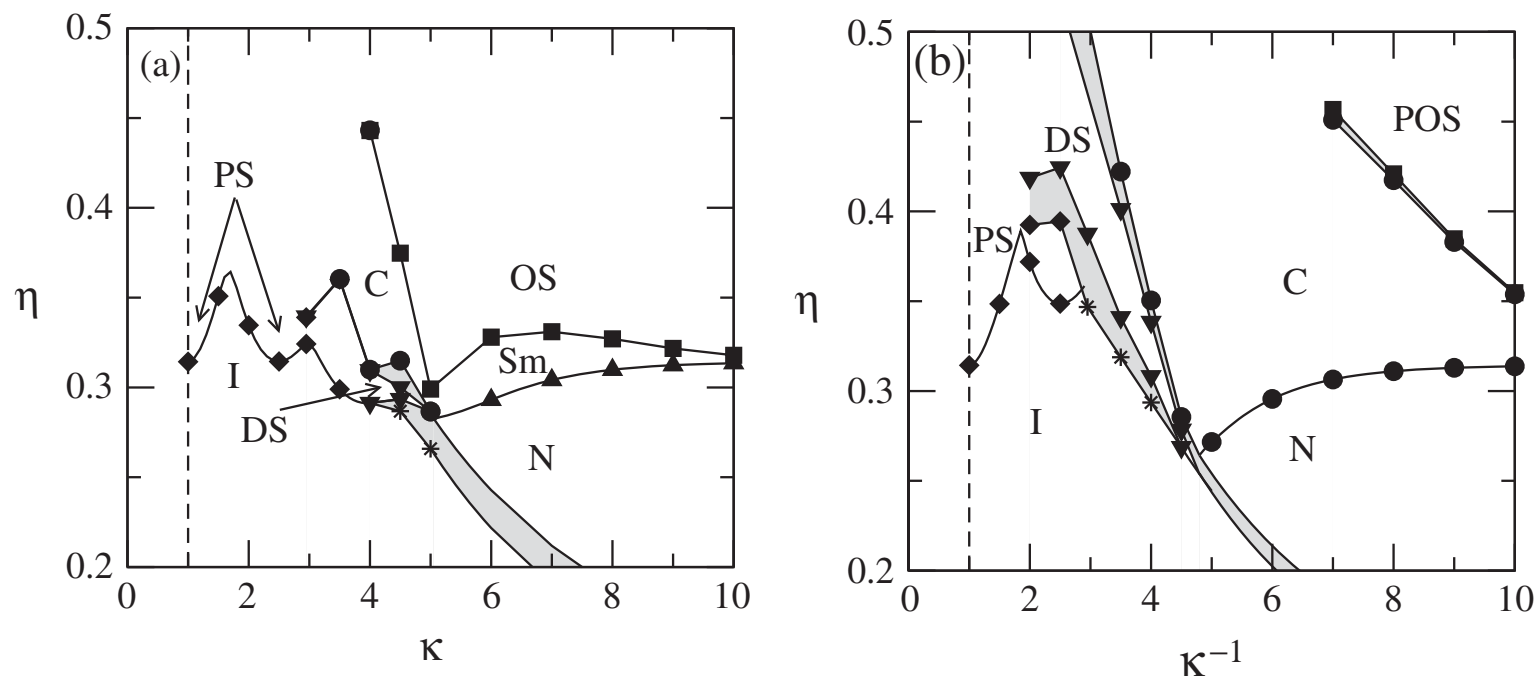

Fig. 7.18. Phase diagrams of prolate (a) and oblate (b) parallelepipeds. Several phases are labeled as I: isotropic, N: nematic, Sm: smectic, DS: discotic smectic, C: columnar, PS: plastic solid, OS: orientationally ordered solid and POS: perfectly orientationally ordered solid. The transition densities are labeled with different symbols. The shaded areas limit the regions of two-phase coexistence 
model [183]. We should say that although the restriction of orientations seems to be a crude simplification to treat the orientational degrees of freedoms, all the liquid crystal phases (smectic, columnar, plastic and oriented solid) found in the theoretical and simulation studies of freely rotating hard particles are present in the Zwanzig phase diagram obtained from FMT. Also, the characteristic aspect ratios at which the smectic and columnar phases of rods and plates become stable $\left(\kappa_{r}=\kappa_{p}^{-1} \sim 5\right)$ are similar to those found in simulations of $\operatorname{HSCs}(\kappa \sim 4.5)[184]$ and cut spheres $\kappa \sim 0.2[185]$.

\section{Rod-plate Polydisperse Mixture}

The second virial approximation of the free energy of the Zwanzig model has been employed to investigate the phase diagram of symmetric mixtures of rods and plates [186]. Stimulated by theoretical calculations made in the early 1970s [187] which show that a binary mixture of rods and plates can stabilize a biaxial nematic phase (in which the symmetry axes of particles of different types point along mutually perpendicular directions), van Roij and Mulder studied the relative stability of this phase against a $\mathrm{N}-\mathrm{N}$ phase separation [186]. The FMT of the Zwanzig model has been applied to study the effect of polydispersity on the stability of the biaxial nematic phase in a mixture of plates and rods $[188,189]$. The polydispersity was introduced in the particle aspect ratio $\kappa=L / \sigma$ (with $L$ and $\sigma$ the length and width of the parallelepiped) around two values $\kappa_{p}<1$ and $\kappa_{r}>1$ symmetrically positioned with respect to $\kappa=1$. It was shown that enough amount of polydispersity can stabilize the biaxial nematic phase even for values of $\kappa_{p}$ and $\kappa_{r}$, for which the bidisperse mixture does not exhibit this phase [188, 189]. In Fig. 7.19(a), we show the phase diagram corresponding to a binary mixture of rods and plates with the same particle volumes and with $\left(\kappa_{r}, \kappa_{p}\right)=(5,0.2)$, while Fig. 7.19 (b) represents the phase diagram of a polydisperse binary mixture with length and breath polydispersities equal to $\Delta_{L}=0.610$ and $\Delta_{\sigma}=0.302$, respectively. As we can see, enough polydispersity can stabilize the biaxial nematic phase [the shaded region in Fig. 7.19(b)], which is not stable in the binary mixture.

Onsager's theory applied to the mixture of freely rotating plates and rods also confirms the presence of the biaxial nematic phase [190, 191]. However, the inclusion of end-effects in the expressions for the excluded volumes destabilizes the biaxial phase with respect to $\mathrm{I}-\mathrm{N}$ or $\mathrm{N}-\mathrm{N}$ phase transitions [192]. There are also several studies employing the extension of the Parsons-Lee theory to a mixture of freely rotating rods and plates $[193,194,195]$. The aim of these works was to shed some light on the very rich phase behavior of the polydisperse rod-plate colloidal mixtures observed in experiments, in which the system can exhibit triphasic equilibrium between phases with different symmetries [196]. The theoretical works predicted different demixing scenarios, some of them in qualitative agreement with experiments $[193,194$, 195]. The Parsons-Lee approach for a binary mixture of rods was later extended by Cinacchi et al. to take into account also smectic inhomogeneities 

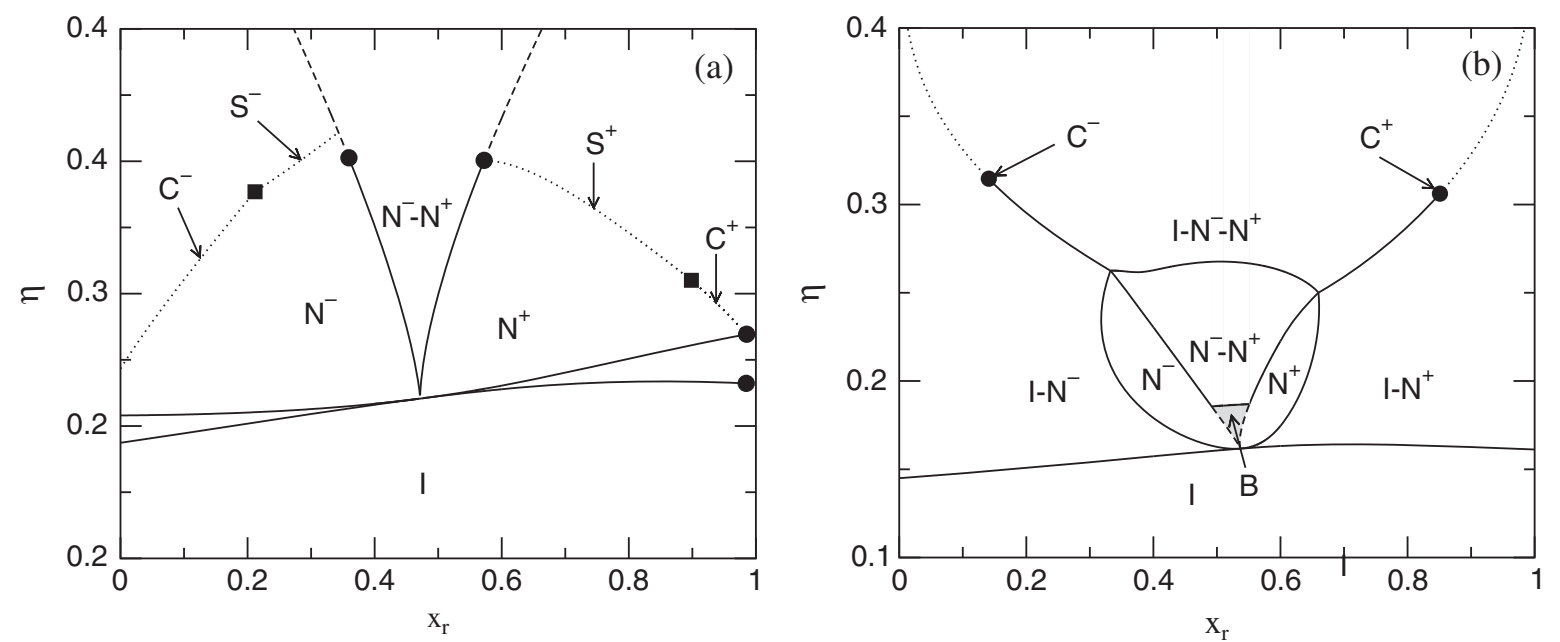

Fig. 7.19. (a): Phase diagram of a binary mixture of rods and plates with $\kappa_{r}=$ $\kappa_{p}^{-1}=5 ; x_{r}$ is the fraction of rods. Phases are labeled I: isotropic, $\mathrm{N}^{-}$: nematic phase of plates, $\mathrm{N}^{+}$: nematic phase of rods, $C^{ \pm}$and $\mathrm{S}^{ \pm}$: columnar and smectic phases of rods $(+)$ and plates $(-) \cdot \mathrm{N}^{-}-\mathrm{N}^{+}$represents the two-phase coexistence region. (b): Phase diagram of polydisperse mixture of rods and plates with the same aspect ratio and length and breath polydispersities $\Delta_{L}=0.610$ and $\Delta_{\sigma}=0.302 . \mathrm{I}^{-\mathrm{N}^{ \pm}}$and $\mathrm{I}-\mathrm{N}^{-}-\mathrm{N}^{+}$represent the two-phase and three-phase coexistence regions. The shaded area shows the region of stability of the biaxial phase (B)

$[197,198,199]$. The main results of their numerical minimization of the proposed functional provided phase diagrams including demixing phase transitions between different smectics and also between smectic and isotropic or nematic phases. For mixtures with species having very different lengths, they found mesophases in which the shortest component is microsegregated at the interstitials between the layers mainly formed by the large component $[197,198,199]$.

\section{Interfacial Phase Behavior}

The treatment of the spatial degrees of freedoms given by the FMT of the Zwanzig model is very accurate, as it was shown by Bier et al. in their theoretical study of the isotropic-nematic interface of a binary mixture of oblate parallelepipeds [200]. Van Roij et al. studied the interface of the onecomponent HR fluid confined in a slit using the Zwanzig model in the limit of infinite elongation, i.e. with the excess part of the free energy density equal to $\Phi_{\mathrm{ex}}=\boldsymbol{n}_{1} \cdot \boldsymbol{n}_{2}[201,202]$. Despite the simplicity of the model, the obtained results are in qualitative agreement with $\mathrm{MC}$ simulations where the confined fluid exhibits a biaxial nematic phase close to the walls whose length increases with the bulk chemical potential up to a value at which a capillary nematization of the pore occurs. This surface phase transition ends in a critical point (a critical value for the pore width) [201, 202]. Later, Harnau and Dietrich extended this study to a binary mixture of HR by applying the Zwanzig model of hard parallelepipeds confined in a slit geometry or in geometrically structured 
surfaces. See their recent review [203] for a compilation of works on the study of liquid crystal interfaces, specifically those using the Zwanzig approach, for particles with prolate and oblate symmetries.

The 2D wall-fluid interface of the HR fluid was recently studied by the 2D version of the FMT for hard rectangles with restricted orientations, Eq. (7.195) and [204]. It was found that for a particular case of hard rectangles having aspect ratio $\kappa=3$, complete wetting by the columnar phase of the wall-isotropic interface occurs. The isotropic fluid confined by two hard lines also exhibits capillary ordering and layering transitions in analogy with the phenomenology found in the study of the confined 3D smectic phase [205, 206]. We show in Fig. 7.20 the interfacial phase diagram (the pore width $H$ versus chemical potential $\mu$ ) obtained from the DF minimization. The figure shows that for chemical potentials well bellow its bulk coexistence value, the confined fluid exhibits a first-order transition to an interfacial phase with columnar symmetry. The 2D columnar phase is a layered phase with the long particle axes parallel to the layers. In Fig. 7.21, we plot the coexisting isotropic phase and the 25 layered columnar phase confined in a pore with $H / \sigma=30$.

For a fixed value of the pore width $H$ and increasing the chemical potential above its bulk coexisting value, an $n-1 \rightarrow n$ layering transition occurs. The capillary ordering and layering transitions coalesce in a set of triple points, two of them shown in Fig. 7.20. The same phase diagram topology was found in MC simulations of a confined hard-sphere fluid [207].

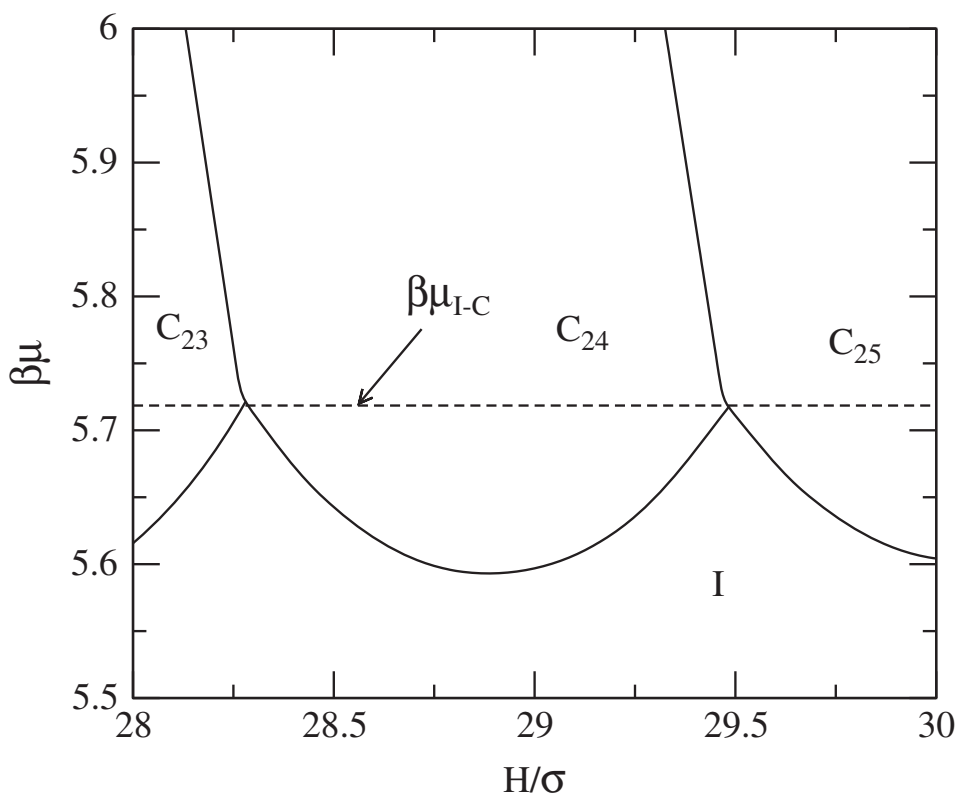

Fig. 7.20. Surface phase diagram, chemical potential $\mu$ versus pore width $H$, of a confined hard rectangle fluid. The solid lines show the capillary isotropic (I)-columnar (C) and $n-1 \rightarrow n$ layering transitions bellow and above the bulk chemical potential $\mu_{I C}$, respectively. The regions of stability of the isotropic and columnar interfacial phases with 23, 24 and 25 layers are shown 

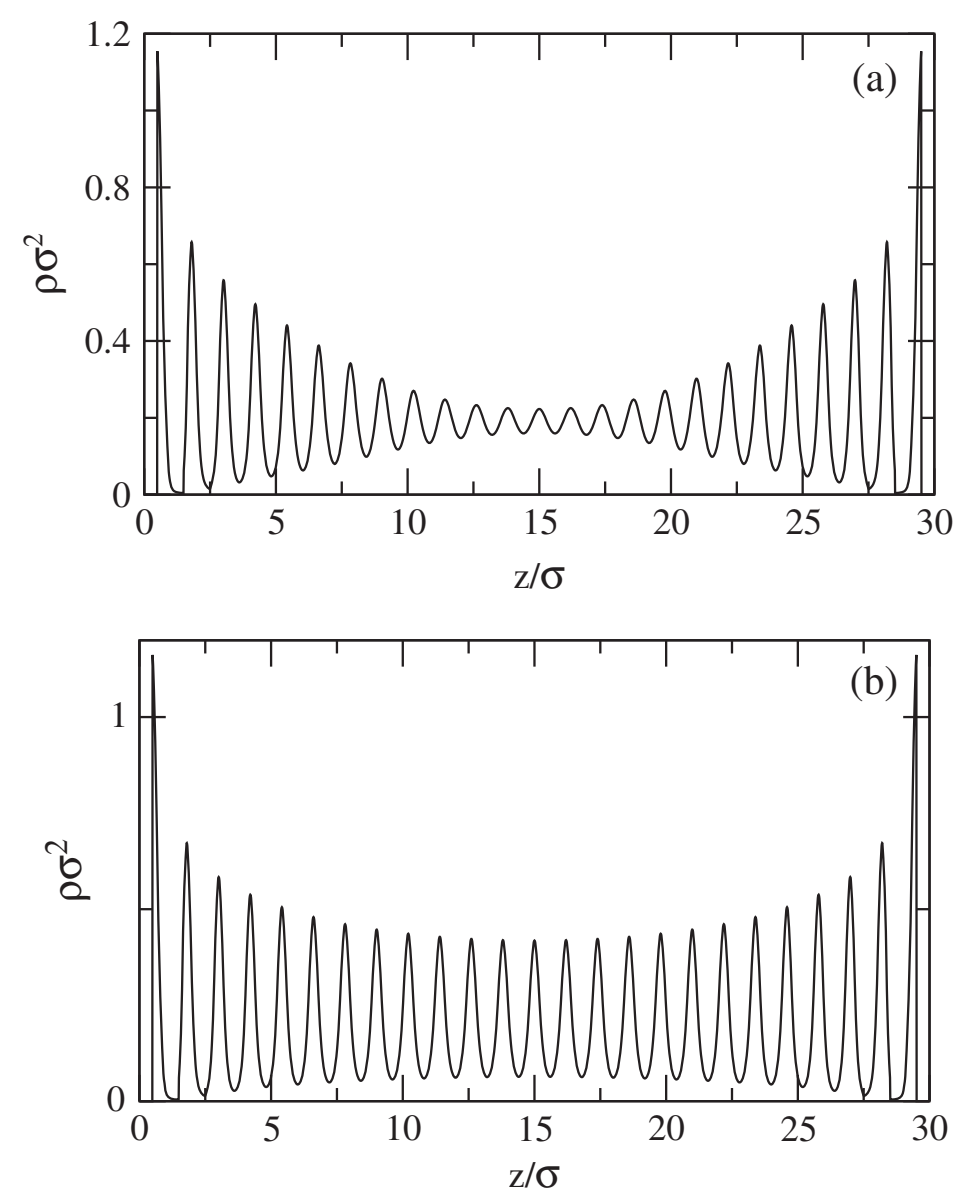

Fig. 7.21. Density profiles of the coexisting isotropic (a) and columnar (b) interfacial phases

\subsubsection{Fundamental Measure Theory for Freely Rotating Hard Anisotropic Particles}

This subsection is devoted to present the recent advances of the extension of the FMT to freely rotating particles. Cinacchi and Schmid proposed a DF approximation for general anisotropic particles interpolating between Rosenfeld's DF for HS and Onsager's DF for HR [208]. However, the first attempt to construct a DF with the use of the exact deconvolution of the Mayer function was made by Schmidt for a mixture of HS and infinitely thin HR [209]. In this model, the interaction between the spheres and needles is also hard, i.e. the pair interaction potential is zero if they do not overlap and is infinite otherwise. Finally, the needles do not interact with each other. The DF is constructed in such a way to recover the exact 0D limit for this model, which has the form $\Phi^{(0)}=\left(1-\eta-\eta^{\prime}\right) \ln (1-\eta)+\eta$, with $\eta$ and $\eta^{\prime}$ the packing fractions of the hard (spheres) and ideal (needles) particles, respectively [209]. Also, the low-density limit of the corresponding functional (the second virial term) has the correct form. Schmidt showed that the Mayer function between a needle and a sphere can be calculated exactly as

$$
f_{\mathrm{sn}}(\boldsymbol{r}, \hat{\boldsymbol{\Omega}})=w_{\mathrm{s}}^{(3)} * w_{\mathrm{n}}^{(0)}(\boldsymbol{r}, \hat{\boldsymbol{\Omega}})+w_{\mathrm{sn}}^{(2)} * w_{\mathrm{n}}^{(1)}(\boldsymbol{r}, \hat{\mathbf{\Omega}}),
$$


where the subindices $\mathrm{s}$ and $\mathrm{n}$ stand for spheres and needles, respectively. The weights corresponding to HS are the same as those of the original FMT (7.179)-(7.181) with the spherical symmetry while those for the needles correspond to the Rosenfeld extension (7.179)-(7.181)

$$
\begin{aligned}
& w_{\mathrm{n}}^{(1)}(\boldsymbol{r}, \hat{\mathbf{\Omega}})=\frac{1}{4} \int_{-L / 2}^{L / 2} \mathrm{~d} l \delta(\boldsymbol{r}+\hat{\mathbf{\Omega}} l), \\
& w_{\mathrm{n}}^{(0)}(\boldsymbol{r}, \hat{\boldsymbol{\Omega}})=\frac{1}{2}[\delta(\boldsymbol{r}+\hat{\mathbf{\Omega}} L / 2)+\delta(\boldsymbol{r}-\hat{\mathbf{\Omega}} L / 2)],
\end{aligned}
$$

except the new one

$$
w_{\mathrm{sn}}^{(2)}(\boldsymbol{r}, \hat{\boldsymbol{\Omega}})=2\left|\boldsymbol{v}_{\mathrm{s}}^{(2)}(\boldsymbol{r}) \cdot \hat{\mathbf{\Omega}}\right|,
$$

which depends on the position of the sphere and on the orientation of the needle. In this sense, this is not strictly a one-particle weight, but its definition is necessary to recover the Mayer function decomposition exactly. The functional for the sphere-needle fluid proposed in [209] has the form $\Phi=\Phi_{\mathrm{s}}+\Phi_{\mathrm{n}}$, with $\Phi_{\mathrm{S}}$ the one-component HS free energy density (7.69)-(7.72), while $\Phi_{\mathrm{S}}$ includes the interaction between spheres and needles and it has the form

$$
\Phi_{\mathrm{sn}}=-n_{\mathrm{n}}^{(0)} \ln \left(1-\eta_{\mathrm{s}}\right)+\frac{n_{\mathrm{n}}^{(1)} n_{\mathrm{sn}}^{(2)}}{1-\eta_{\mathrm{s}}}
$$

where $n_{\mu}^{(\alpha)}(\boldsymbol{r}, \hat{\boldsymbol{\Omega}})=\rho_{\mu} * w_{\mu}^{(\alpha)}(\boldsymbol{r}, \hat{\boldsymbol{\Omega}})$ for $\mu=\mathrm{n}$, sn, while $\eta_{\mathrm{s}}(\boldsymbol{r})$ is the HS local packing fraction. Thus, the excess free energy is calculated as $\beta \mathcal{F}_{\text {ex }}\left[\rho_{\mathrm{s}}, \rho_{\mathrm{n}}\right]=$ $\int \mathrm{d} \boldsymbol{x} \Phi\left(\left\{n_{\alpha}^{(\beta)}\right\}\right)$, where the integration is taken over $\boldsymbol{x}=\{\boldsymbol{r}, \hat{\boldsymbol{\Omega}}\}$, the spatial and angular variables. This functional was applied to the calculation of the bulk phase diagram of the isotropic rod-sphere mixture for different values of the aspect ratio $L / \sigma$. The phase diagrams are identical to those obtained from the free-volume theory, which predicts a demixing transition ending in critical points. The direct sphere-sphere correlation function obtained from the DF is in good agreement with simulations.

To include the rod-rod interaction in the theory, Brader et al. expressed the Mayer function between two needles as the following convolution

$$
f_{\mathrm{nn}}\left(\boldsymbol{r}, \hat{\mathbf{\Omega}}, \hat{\mathbf{\Omega}}^{\prime}\right)=w_{\mathrm{nn}}^{(2)}\left(\boldsymbol{r}, \hat{\mathbf{\Omega}} ; \hat{\mathbf{\Omega}}^{\prime}\right) * w_{\mathrm{n}}^{(1)}\left(\boldsymbol{r}, \hat{\mathbf{\Omega}}^{\prime}\right),
$$

where the new weight

$$
w_{\mathrm{nn}}^{(2)}\left(\boldsymbol{r}, \hat{\mathbf{\Omega}} ; \hat{\mathbf{\Omega}}^{\prime}\right)=16 D \sqrt{1-\left(\hat{\boldsymbol{\Omega}} \cdot \hat{\mathbf{\Omega}}^{\prime}\right)^{2}} w_{\mathrm{n}}^{(1)}(\boldsymbol{r}, \hat{\mathbf{\Omega}})
$$

was introduced to describe the residual rod surface in the limit of large aspect ratios [210]. Note that the new weight $w_{\mathrm{nn}}^{(2)}$ depends on the orientations of both needles. Thus, the new weighted density is calculated as 


$$
n_{\mathrm{nn}}^{(2)}(\boldsymbol{r}, \hat{\mathbf{\Omega}})=\int \mathrm{d} \hat{\mathbf{\Omega}}^{\prime} \rho_{\mathrm{n}}\left(\boldsymbol{r}, \hat{\mathbf{\Omega}}^{\prime}\right) * w_{\mathrm{nn}}^{(2)}\left(\boldsymbol{r}, \hat{\mathbf{\Omega}}^{\prime} ; \hat{\mathbf{\Omega}}\right),
$$

where, apart from the spatial convolution, the angular average with respect to the angles defining the unit vector $\hat{\boldsymbol{\Omega}}^{\prime}$ is taken. This fact complicates the calculations involved in the excess free energy evaluation. The new form of the excess free energy density now becomes $\Phi=\Phi_{\mathrm{s}}+\Phi_{\mathrm{sn}}+\Phi_{\mathrm{snn}}$, with

$$
\Phi_{\mathrm{snn}}=\frac{n_{\mathrm{n}}^{(1)} n_{\mathrm{nn}}^{(2)}}{1-\eta_{\mathrm{s}}} .
$$

This functional was applied to study the planar fluid-fluid interface of the HS HR mixture. The sphere and needle density profiles show either monotonic or damped oscillatory behavior on the sphere-rich side of the interface, depending on which side of the Fisher-Widom line is the bulk fluid in the phase diagram for pure HS. On the needle-rich side, both density profiles decay monotonically toward their respective bulk densities. Needles have biaxial order (they lie preferentially parallel to the interface) on the needle-rich side, while on the sphere-rich side they are oriented normal to the interface [210].

A further extension of the sphere-needle functional was made by Esztermann and Schmidt to account for the first correction (of order $D / L$ ) to the Mayer function decomposition between two HSCs [211]. This is accomplished by introducing four new geometric weight functions into the framework. Their corresponding weighted densities, $\tilde{n}_{i}$, combine in the new contribution $\Delta \Phi=f\left(\left\{\tilde{n}_{i}\right\}\right) /\left(1-\eta_{\mathrm{s}}\right)$ to the excess free energy density, $\Phi=$ $\Phi_{\mathrm{s}}+\Phi_{\mathrm{sn}}+\Phi_{\mathrm{snn}}+\Delta \Phi$, with $\left\{\tilde{n}_{i}\right\}$, the set of new introduced weighted densities. See the details in [211].

The same formalism was extended to propose a FMT for a ternary mixture of HS, hard platelets and HR, with both needles and platelets of vanishing thickness [212]. The geometric weights were constructed to ensure the exact decomposition of the Mayer functions between different species, except that corresponding to the sphere-platelet interaction, for which the same level of approximation proposed by Rosenfeld for the HD fluid was used. The functional in the one-component platelet limit was tested to consider the isotropicnematic bulk transition, which was found to be weakly first order, with values for the coexistence densities and the nematic order parameter that compare well with simulation results [212].

\subsection{Summary}

In this chapter, we have presented a review of DF theory, one of the most important developments of the theory of liquids, whose history is strongly entangled to that of the HS model. Beginning with a general description of the conceptual framework of the theory, we have presented the most important approximations developed to describe the thermodynamics and structure of 
the HS fluid. Rosenfeld's FMT has been given a special treatment both because of its more involved structure and because it nowadays stands as the most successful DF approximation. The perspective of the advances made during the past two decades is certainly impressive: in the early 1980s, the LDA was nearly the only available choice for the treatment of the HS packing effects within the DF formalism, and now we have DF approximations which may extract from $\rho(\boldsymbol{r})$ the discreteness of the particles, to give the exact 0D limit in a narrow cavity. Despite such impressive progress, there are challenges ahead, which are probably beyond the scope of the FMT. The consistent improvement over the PY equation of state for the bulk fluid, but keeping all the advantages of the DI-FMT for the crystal phase, would be a most important goal for the future.

Beyond the simplest mono-component HS model, we have considered two extensions: mixtures of HS and anisotropic hard convex bodies. The reason is that most approximations initially developed for HS have been later generalized, with more or less success, in these two directions. Again, FMT plays a crucial role in the extension to mixtures, and it has been applied not only to ordinary additive mixtures but also to non-additive ones, of particular interest in the theory of colloids and polymers. The superiority of the FMT with respect to any other DF approximation is absolute for these systems. Before its publication, only binary mixtures were amenable to the DF formalism and even then with great difficulty. FMT has permitted to study multicomponent mixtures, as well as polydisperse systems, with only a manageable level of difficulty. But it is also for mixtures that the limits of the theory become more evident. Future workers in the field have plenty of opportunities to develop better DF approximations for HS mixtures of very different size and to deal with the effects of non-additivity of their excluded cores.

Finally, we have described two alternative approaches for a problem which is qualitatively more difficult than the HS packing: the construction of density functionals for hard anisotropic bodies, beyond the generic low-density expansion pioneered by Onsager. The first route is to try the approximate assembly of the excellent DF for isotropic hard cores, with those magnitudes characterizing the anisotropic pair interactions (such as the Mayer function or the contact distance). The second approach is based on the extension of the FMT to these systems, as it was already attempted by Rosenfeld, so that the crucial ingredient for the non-local dependence of the free energy DF would be the shape of a single molecule rather than the excluded volume between two molecules. The approach has been very successful for systems of parallel anisotropic bodies, in which the orientational degrees of freedom are frozen. A partial extension to systems with orientational disorder has been done within Zwanzig model for liquid crystals: hard parallelepipeds with restricted orientations, which may be treated as a mixture of perfectly oriented bodies. At this point, an interesting question arises: What kind of particle geometry is suitable for first principles derivation of the fundamental measure functional? A useful criterion requires that the pair overlap volume between particles 
should have the same symmetry of constituent particles. This requirement is fulfilled for HS and hard parallelepipeds, but it does not apply to freely rotating anisotropic bodies. This appears to be the fundamental reason why the recent extensions of FMT to freely rotating anisotropic particles are forced to make strong simplifications, like an expansion in the limit of large particle anisotropy. Our only certainty here is that the field is open and game goes on!

\section{References}

1. R. Evans: Avd. Phys. A 28, 143 (1979)

2. R. Evans: Density functionals on the theory of nonuniform fluids. In Fundamentals of Inhomogeneous Fluids, ed by D. Henderson (Dekker, New York 1992) pp 85.

3. H. Löwen: J. Phys.: Cond. Matter 14, 11897 (2002)

4. J. K. Percus: J. Stat. Phys. 15, 505 (1976)

5. J.-P. Hansen and I. R. McDonald: Theory of simple liquids, 2nd edn (Academic Press, London 1990) p 39

6. P. Tarazona and R. Evans: Mol. Phys. 47, 1033 (1982)

7. J. S. Rowlinson and B. Widom: Molecular Theory of Capillarity (Oxford University Press, Oxford 1989) (reprinted by Dover, New York 2002)

8. A. A. Louis, P. G. Bolhuiss, and J.-P. Hansen: Phys. Rev. E 62, 7961 (2000)

9. S. Torquato: Random Heterogenous Materials (Springer, New York 2001) p 81

10. A. Robledo: J. Chem. Phys. 72, 1701 (1980)

11. A. Robledo and C. Varea: J. Stat. Phys. 28, 513 (1980)

12. Y. Rosenfeld: J. Chem. Phys. 89, 4272 (1988)

13. Y. Rosenfeld: Phys. Rev. Lett. 63, 980 (1989)

14. S. Nordholm, M. Johnson and B. C. Freasier: Aust. J. Chem. 33, 2139 (1980)

15. O. Gunnarsson, M. Johnson, and B.I. Lundqvist: Phys. Rev. B 20, 3136 (1979).

16. P. Tarazona: Mol. Phys. 52, 81 (1984)

17. P. Tarazona: Phys. Rev. A 31, 2672 (1985)

18. W. A. Curtin and N. W. Ashcroft: Phys. Rev. A 32, 2909 (1985)

19. P. Tarazona, U. Marini Bettolo Marconi and R. Evans: Molec. Phys. 60, 573 (1987)

20. W. H. Press, B. P. Flannery, S. A. Teukolsky, W. T. Vetterling: Numerical Recipes in FORTRAN: The Art of Scientific Computing (Cambridge University Press, Cambridge 1992)

21. I. K. Snook, and D. Henderson: J. Chem. Phys. 68, 2134 (1978)

22. J. R. Henderson and F. van Swol: Mol. Phys. 51, 991 (1984)

23. H. Reis, H. Frisch and J. L. Lebowitz: J. Chem. Phys. 31, 369 (1959)

24. H. Reis, H. Frisch, E. Helfand and J. L. Lebowitz: J. Chem. Phys. 32, 119 (1960)

25. A. Papadopoulou, F. van Swol and U. Marini Bettolo Marconi: J. Chem. Phys. 976942 (1992)

26. S.-C. Kim, J.-K. Suh and S.-H. Suh: Mol. Phys. 79, 1369 (1993)

27. G. P. Brenan and R. Evans: Mol Phys. 73, 789 (1991)

28. E. Velasco and P. Tarazona: J. Chem. Phys. 91, 7916 (1989) 
29. P. Röcken, A. Somoza, P. Tarazona and G. Findenegg: J. Chem. Phys. 108, 8689 (1998)

30. B. J. Alder, T. E. Wainwright: J. Chem. Phys. 33, 1439 (1960)

31. W. G. Hoover and F. H. Ree: J. Chem. Phys. 49, 3609 (1968)

32. B. J. Alder, W. G. Hoover and D.A. Young: J. Chem. Phys. 49, 3688 (1968)

33. D. A. Young and B. J. Alder: J. Chem. Phys. 60, 1254 (1974)

34. H. Löwen: Phys. Rep. 237249 (1994)

35. T. V. Ramakrishnan: Phys. Rev. Lett. 48541 (1982)

36. M. Yussouff: Phys. Rev. B 235871 (1981)

37. A. D. J. Haymet: J. Chem. Phys. 784641 (1983)

38. R. Ohnesorge, H. Löwen and H. Wagner: Phys. Rev. E 50, 4801 (1994)

39. E. Velasco, P. Tarazona: Phys. Rev. A 36, 979 (1987)

40. M. Jaric, U. Mohanty: Phys. Rev. Lett. 58, 230 (1987)

41. M. Takamiya and K. Nakanishi: Mol. Phys. 70, 767 (1990)

42. E. Velasco and L. Mederos: Phys. Rev. E 56, 2433 (1997)

43. L. Mederos, G. Navascués, P. Tarazona and E. Chacón: Phys. Rev. E 47, 4284 (1993).

44. A. de Kuijper, W. L. Vos, J.-L. Barrat, J.-P. Hansen and J. A. Schouten: J. Chem. Phys. 93, 5187 (1990)

45. B. B. Laird and D.M. Kroll: Phys. Rev. A 42, 4810 (1990)

46. R. Groot: J. Chem. Phys. 95, 9191 (1990)

47. T.F. Meister and D.M. Kroll: Phys. Rev. A 31, 4155 (1985)

48. R.D. Groot and J.P. van Eerden, Phys. Rev. A 36, 4356 (1987)

49. A. R. Denton and N. W. Ashcroft: Phys. Rev. A 39, 4701 (1989)

50. A. R. Denton and N. W. Ashcroft: Phys. Rev. A 42, 7312 (1990)

51. A. Khein and N.W. Ashcroft: Phys. Rev. Lett. 78, 3346 (1997)

52. M. Baus: J. Phys. Conds. Matter. 1, 3131 (1989)

53. J.F. Lutsko and M. Baus: Phys. Rev. Lett. 64, 761 (1990)

54. P. Tarazona and Y. Rosenfeld: Phys. Rev. E 55, R4873 (1997)

55. B. Barboy and W. M. Gelbart: J. Stat. Phys. 22, 685 (1980)

56. E. Kierlik and M.-L. Rosinberg: Phys. Rev. A 42, 3382 (1990)

57. E. Kierlik and M.-L. Rosinberg: Phys. Rev. A 33, 2025 (1986)

58. S. Phan, E. Kierlik, M.-L. Rosinberg, B. Bildstein and G. Kahl: Phys. Rev. E 48, 618 (1993)

59. Y. Rosenfeld: J. Chem. Phys. 98, 8126 (1993)

60. Y. Rosenfeld: Phys. Rev. A 33, 2025 (1986)

61. P. Tarazona: Phys. Rev. Lett. 84, 694 (2000)

62. P. Tarazona: Physica A 306, 243 (2002)

63. J. L. Colot and M. Baus: Phys. Rev. A 36, 3912 (1987)

64. Y. Rosenfeld, M. Schmidt, H. Löwen and P. Tarazona: J. Phys.: Cond. Matter 8, L577 (1997)

65. Y. Rosenfeld, M. Schmidt, H. Löwen and P. Tarazona: Phys. Rev. E 55, 4245 (1999)

66. F. J. Lutsko: Phys. Rev. E 74, 021121 (2006)

67. B. Groh: Phys. Rev. E 61, 5218 (2000)

68. C. Rascón, E. Velasco, L. Mederos and G. Navascués: J. Chem. Phys. 106, 6689 (1997)

69. A. González, F. L. Román and J. A. White: J. Phys.: Condens. Matter 11, 3789 (1999) 
70. A. González, F. L. Román, S. Velasco and J. A. White: J. Chem. Phys. 125 064703 (2006)

71. A. González, J. A. White and R. Evans: J. Phys.: Condens. Matter 9, 2375 (1997)

72. R. Roth, R. Evans, A. Lang and G. Kahl: J. Phys.: Condens. Matter 14, 12063 (2002)

73. T. L. Hill: An introduction to Statistical Thermodynamics (Dover, New York, 1986), pp 177-188

74. J. N. Israelachvili, D. J. Mitchell and B. W. Ninham: J. Chem. Soc. Faraday Trans. II 72, 1525 (1976)

75. R. P. Sear and J. A. Cuesta: Europhys. Lett. 55, 451 (2001)

76. R. Blaak and J. A. Cuesta: J. Chem. Phys. 115, 963 (2001)

77. J. A. Cuesta and R. P. Sear: Phys. Rev. E 65, 031406 (2002)

78. R. L. Davidchack and B. B. Laird: Phys. Rev. E 60, 3417 (1999)

79. A. R. Denton and N. W. Ashcroft: Phys. Rev. A 44, 8242 (1991)

80. S.-C. Kim, C. H. Lee and B. S. Seong: Phys. Rev. E 60, 3413 (1999)

81. T. K. Vanderlick, H. T. Davis and J. K. Percus: J. Chem. Phys. 91, 7136 (1989)

82. I. Pagonabarraga, M. E. Cates and G. J. Ackland: Phys. Rev. Lett. 94, 911 (2000)

83. D. Goulding, S. Melchionna and J.-P. Hansen: Phys. Chem. Chem. Phys. 3, $1644(2001)$

84. J. A. Cuesta, Y. Martínez-Ratón and P. Tarazona: J. Phys.: Condens. Matter 14, 11965 (2002)

85. Y.-X. Yu and J. Wu: J. Chem. Phys. 117, 10156 (2002)

86. Y.-X. Yu, J. Wu, Y.-X. Xin and G.-H. Gao: J. Chem. Phys. 121, 1535 (2004)

87. H. Hansen-Goos and R. Roth: J. Phys.: Condens. Matter 18, 8413 (2006)

88. R. K. Bowles: Physica A 275, 217 (2000)

89. S. Asakura and F. Oosawa: J. Chem. Phys. 22, 1255 (1954)

90. A. Vrij: Pure Appl. Chem. 48, 471 (1976)

91. M. Schmidt, H. Löwen, J. M. Brader and R. Evans: Phys. Rev. Lett. 85, 1934 (2000)

92. B. Götzelmann, R. Roth, S. Dietrich, M. Dijkstra and R. Evans: Europhys. Lett. 47, 398 (1999)

93. J. A. Cuesta and Y. Martínez-Ratón: J. Phys.: Condens. Matter 11, 10107 (1999)

94. M. Schmidt, H. Löwen, J. M. Brader and R. Evans: J. Phys.: Condens. Matter 14, $9353(2002)$

95. H. N. W. Lekkerkerker, W. C.-K. Poon, P. N. Pusey, A. Stroobants and P. B. Warren: Europhys. Lett. 20, 559 (1992)

96. M. Dijkstra, J. M. Brader and R. Evans: J. Phys.: Condens. Matter 11, 10079 (1999)

97. M. Schmidt and A. R. Denton: Phys. Rev. E 65, 061410 (2002)

98. B. Widom and J. S. Rowlinson: J. Chem. Phys. 52, 1670 (1970)

99. M. Schmidt: Phys. Rev. E 63, 010101(R) (2000)

100. R. P. Sear: J. Chem. Phys. 104, 9948 (1996)

101. M. Schmidt, J. Phys.: Condens. Matter 16, L351 (2004)

102. G. Friedel: Ann. Phys. 18, 273 (1922)

103. L. Onsager: Phys. Rev. 62, 558 (1942) 
104. L. Onsager: Ann. N. Y. Acad. Sci. 51, 627 (1949)

105. A. Stroobants, H. N. W. Lekkerkerker and D. Frenkel: Phys. Rev. A 36, 2929 (1987)

106. D. Frenkel: J. Phys. Chem. 91, 4912 (1987)

107. D. Frenkel, H. N. W. Lekkerkerker and A. Stroobants: Nature (London) 332, $882(1988)$

108. W. Maier and A. Saupe: Z. Naturforsch. 13A, 564 (1958)

109. W. Maier and A. Saupe: Z. Naturforsch. 14A, 882 (1959)

110. W. Maier and A. Saupe: Z. Naturforsch. 15A, 287 (1960)

111. M. Abramowitz and I. A. Stegun: Handbook of mathematical functions (Dover, New York 1965)

112. W. L. McMillan: Phys. Rev. A 4, 1238 (1971)

113. J. V. Selinger and D. R. Nelson: Phys. Rev. A 35, 1736 (1988)

114. M. M. Telo da Gama: Mol. Phys. 52, 585 (1984)

115. M. M. Telo da Gama: Mol. Phys. 52, 611 (1984)

116. C. Gray and K. E. Gubbins: Theory of Molecular Fluids (Oxford University Press 1984)

117. L. Mederos and D. E. Sullivan: Phys. Rev. A 39, 854 (1989)

118. A. M. Somoza, L. Mederos and D. E. Sullivan: Phys. Rev. Lett 72, 3674 (1994)

119. A. M. Somoza, L. Mederos and D. E. Sullivan: Phys. Rev. E 52, 5017 (1995)

120. Y. Martínez-Ratón, A. M. Somoza, L. Mederos and D. E. Sullivan: Faraday Discuss. 104, 111 (1996)

121. Y. Martínez-Ratón, A. M. Somoza, L. Mederos and D. E. Sullivan: Phys. Rev. E 55, 2030 (1997)

122. B. J. Berne and P. Pechukas: J. Chem. Phys. 56, 4213 (1972)

123. J. G. Gay and B. J. Berne: J. Chem. Phys. 74, 3316 (1981)

124. E. Velasco, A. M. Somoza and L. Mederos: J. Chem. Phys. 102, 8107 (1995)

125. E. Velasco and L. Mederos: J. Chem. Phys. 109, 2361 (1998)

126. H. N. W. Lekkerkerker, P. Coulon, R. van der Haegen and R. Deblieck: J. Chem. Phys. 80, 3427 (1984)

127. J. D. Parsons: Phys. Rev. A A19, 1225 (1979)

128. S. D. Lee: J. Chem. Phys. 87, 4972 (1987)

129. S. D. Lee: J. Chem. Phys. 89, 7036 (1988)

130. R. M. Gibbons: Mol. Phys. 17, 81 (1969)

131. M. A. Cotter and D. E. Martire: J. Chem. Phys. 52, 1902 (1970)

132. M. A. Cotter and D. E. Martire: J. Chem. Phys. 53, 4500 (1970)

133. M. A. Cotter: Phys. Rev. A 10, 625 (1974)

134. M. A. Cotter: J. Chem. Phys. 66, 1098 (1977)

135. G. J. Vroege and H. N. W. Lekkerkerker: Rep. Prog. Phys. 55, 1241 (1992)

136. K. Shundyak and R. van Roij: J. Phys.: Condens. Matter 13, 4789 (2001)

137. K. Shundyak and R. van Roij: Phys. Rev. Lett 88, 205501 (2002)

138. K. Shundyak and R. van Roij: Phys. Rev. E 68, 061703 (2003)

139. M. D. Lipkin and D. W. Oxtoby: J. Chem. Phys. 79, 1939 (1983)

140. U. P. Singh and Y. Singh: Phys. Rev. A 33, 2725 (1986)

141. J. F. Marko: Phys. Rev. Lett. 60, 325 (1988)

142. J. F. Marko: Phys. Rev. A 39, 2050 (1989)

143. R. Pynn: Solid State Commun. 14, 29 (1974)

144. R. Pynn: J. Chem. Phys. 60, 4579 (1974)

145. M. Baus: J. Stat. Phys. 48, 1129 (1987) 
146. J. L. Colot, X. G. Wu, H. Xu and M. Baus: Phys. Rev. A 38, 2022 (1988)

147. A. M. Somoza and P. Tarazona: Phys. Rev. Lett 61, 2566 (1988)

148. A. M. Somoza and P. Tarazona: J. Chem. Phys. 91, 517 (1989)

149. A. M. Somoza and P. Tarazona: Phys. Rev. A. 40, 4161 (1989)

150. A. Poniewierski and R. Holyst: Phys. Rev. Lett. 61, 2461 (1988)

151. R. Holyst and A. Poniewierski: Mol. Phys. 68, 381 (1989)

152. E. Velasco, L. Mederos and D. E. Sullivan: Phys. Rev. E 62, 3708 (2000)

153. Y. Rosenfeld: Phys. Rev. E 50, R3318 (1994)

154. A. Pressley: Elementary Differential Geometry (Springer 2001)

155. A. Chamoux and A. Perera: J. Chem. Phys. 104, 1493 (1996)

156. J. A. Cuesta: Phys. Rev. Lett. 76, 3742 (1996)

157. J. A. Cuesta and Y. Martínez-Ratón: Phys. Rev. Lett. 78, 3681 (1997)

158. J. A. Cuesta, Y. Martínez-Ratón: J. Chem. Phys. 107, 6379 (1997)

159. L. Lafuente and J. A. Cuesta: Phys. Rev. Lett. 89, 145701 (2002)

160. L. Lafuente and J. A. Cuesta: J. Phys.: Condens. Matter 14, 12079 (2002)

161. L. Lafuente and J. A. Cuesta: J. Chem. Phys. 119, 10832 (2003)

162. L. Lafuente and J. A. Cuesta: Phys. Rev. E 68, 066120 (2003)

163. L. Lafuente and J. A. Cuesta: Phys. Rev. Lett. 93, 130603 (2004)

164. L. Lafuente and J. A. Cuesta: J. Phys. A: Math. Gen. 38, 7461 (2005)

165. J. A. Capitán and J. A. Cuesta: Phys. Rev. E 76, 011403 (2007)

166. Y. Martínez-Ratón and J. A. Cuesta: J. Chem. Phys. 111, 317 (1999)

167. F. van Swol and V. Woodcock: Mol. Simul. 1, 95 (1987)

168. E. A. Jagla: Phys. Rev. E 58, 4701 (1998)

169. H. Schlacken, H.-J. Moegel and P. Schiller: Mol. Phys. 93, 777 (1998)

170. Y. Martínez-Ratón, E. Velasco and L. Mederos: Phys. Rev. E 72, 031703 (2005)

171. Y. Martínez-Ratón, E. Velasco and L. Mederos: J. Chem. Phys. 125, 014501 (2006)

172. A. Donev, J. Burton, F. H. Stillinger and S. Torquato: Phys. Rev. B 73, 054109 (2006)

173. D. de las Heras, Y. Martínez-Ratón and E. Velasco: Phys. Rev. E 76, 031704 (2007)

174. Y. Martínez-Ratón and J. A. Cuesta: Phys. Rev. E 58, R4080 (1998)

175. J. A. Cuesta and Y. Martínez-Ratón: J. Phys.: Condens. Matt. 12, A109 (2000)

176. M. Dijkstra, R. Van Roij and R. Evans: Phys. Rev. Lett. 81, 2268 (1998)

177. M. Dijkstra, R. Van Roij and R. Evans: 82, 117 (1999)

178. M. Dijkstra, R. Van Roij and R. Evans: Phys. Rev. E 59, 5744 (1999)

179. N. G. Almarza and E. Enciso: Phys. Rev. E 59, 4426 (1999)

180. R. W. Zwanzig: J. Chem. Phys. 24, 855 (1956)

181. R. W. Zwanzig: J. Chem. Phys. 39, 1714 (1963)

182. Y. Martínez-Ratón: Phys. Rev. E 69, 061712 (2004)

183. A. Casey and P. Harrowell: J. Chem. Phys. 103, 6143 (1995)

184. P. Bolhuis and D. Frenkel: J. Chem. Phys. 106, 666 (1997)

185. J. A. C. Veerman and D. Frenkel: Phys. Rev. A 45, 5632 (1992)

186. R. van Roij and B. Mulder: J. Phys. II France 4, 1763 (1994)

187. R. Alben: J. Chem. Phys. 59, 4299 (1973)

188. Y. Martínez-Ratón and J. A. Cuesta: Phys. Rev. Lett. 89, 185701 (2001)

189. Y. Martínez-Ratón and J. A. Cuesta: J. Chem. Phys. 118, 10164 (2003) 
190. H. H. Wensink, G. J. Vroege and H. N. Lekkerkerker: Phys. Rev. E 66, 041704 (2002)

191. S. Varga, A. Galindo and G. Jackson: Phys. Rev. E 66, 011707 (2002)

192. S. Varga, A. Galindo and G. Jackson: J. Chem. Phys. 117, 10412 (2002)

193. H. H. Wensink, G. J. Vroege and H. N. Lekkerkerker: J. Chem. Phys. 115, 7319 (2001)

194. S. Varga, A. Galindo and G. Jackson: J. Chem. Phys. 117, 7207 (2002)

195. S. Varga, A. Galindo and G. Jackson: Mol. Phys. 101, 817 (2003)

196. F. M. van der Kooij and H. N. W. Lekkerkerker: Phys. Rev. Lett. 84, 781 (2000)

197. G. Cinacchi, E. Velasco and L. Mederos: J. Phys. Condens. Matt. 16, S2003 (2004)

198. G. Cinacchi, E. Velasco and L. Mederos: J. Chem. Phys. 121, 3854 (2004)

199. G. Cinacchi, Y. Martínez-Ratón, L. Mederos and E. Velasco: J. Chem. Phys. 124, $234904(2006)$

200. M. Bier, L. Harnau and S. Dietrich: Phys. Rev. E 69, 021506 (2004)

201. R. van Roij, M. Dijkstra and R. Evans: J. Chem. Phys. 113, 7689 (2000)

202. M. Dijkstra, R. Van Roij and R. Evans: Phys. Rev. E 63, 051703 (2001)

203. L. Harnau and S. Dietrich: Soft Matter 3, 156 (2007)

204. Y. Martínez-Ratón: Phys. Rev. E 75, 051708 (2007)

205. D. de las Heras, E. Velasco and L. Mederos: Phys. Rev. Lett. 94, 017801 (2005)

206. D. de las Heras, E. Velasco and L. Mederos: Phys. Rev. E 70, 011709 (2006)

207. A. Fortini and M. Dijkstra: J. Phys. Condens. Matter 18, L371 (2006)

208. G. Cinacchi and F. Schmid: J. Phys.: Condens. Matter 14, 12223 (2002)

209. M. Schmidt: Phys. Rev. E 63, 050201(R) (2001)

210. J. M. Brader, A. Esztermann and M. Schmidt: Phys. Rev. E 66, 031401 (2002)

211. A. Esztermann and M. Schmidt: Phys. Rev. E 70, 022501 (2004)

212. A. Esztermann, H. Reich and M. Schmidt: Phys. Rev. E 73, 011409 (2006) 